Atratores para sistemas dinâmicos discretos: dimensão fractal e continuidade da estrutura por perturbações 
SERVIÇO DE PÓS-GRADUAÇÃO DO ICMC-USP

Data de Depósito:

Assinatura:

\title{
Atratores para sistemas dinâmicos discretos: dimensão fractal e continuidade da estrutura por perturbações
}

\author{
Matheus Cheque Bortolan
}

Orientador: Prof. Dr. Hildebrando Munhoz Rodrigues

Dissertação apresentada ao Instituto de Ciências Matemáticas e de Computação - ICMC-USP, como parte dos requisitos para obtenção do título de Mestre em Ciências - Ciências de Computação e Matemática Computacional.

USP - São Carlos

Abril/2009 
"Bom mesmo é ir à luta com determinação, abraçar a vida e viver com paixão, perder com classe e viver com ousadia. Pois o triunfo pertence a quem se atreve, e a vida é muito bela para ser insignificante."

Charles Chaplin 


\section{Agradecimentos}

Inicialmente gostaria de agradecer à Deus e à minha família, que sempre me deram força, coragem e apoio incondicional para eu seguir em frente, mesmo nas horas mais difíceis. Amo demais todos vocês!

Agradeço também à minha mais que namorada, amiga, confidente, Juliana, por sempre estar comigo e ter me ajudado em todos os sentidos para que este trabalho fosse realizado, pela confiança, carinho nos momentos ruins e bons, pelas broncas nas horas certas, conselhos, por dividir sua história comigo, por todo seu amor! Amo-te muito! Obrigado por fazer parte da minha vida!

Agradeço à todos os professores, em especial ao meu orientador prof. Hildebrando e ao prof. Alexandre, por me ajudarem a trilhar este caminho difícil, mas que no final, é compensador. Nada disso teria acontecido sem vocês, muito obrigado!

Agradeço à FAPESP pelo apoio financeiro à este trabalho.

Aos amigos que fiz nessa jornada e que nunca irei esquecer, Moreno P. Bonutti, pelas horas de "estudo" e risadas, pelos almoços e churrascos e por se revelar amigo para todas as horas. Rodrigo Pedra Brum, que muitas vezes deixava de estudar para me ajudar com problemas computacionais e me animava com longas conversas. À turma da sala 4-009, em especial para Fábio, Paulo e Marcos, por seus vários conhecimentos em matemática, e claro em jogos e internet.

Enfim, se for agradecer a cada um, não acabaria nunca, pois são tantas as pessoas especiais que passaram e marcaram esta jornada...

A todos vocês, muitíssimo obrigado!!! 


\section{Resumo}

Neste trabalho, estudamos uma generalização dos semigrupos discretos gradientes, os semigrupos gradient-like, algumas de suas propriedades e a sua invariância por pequenas perturbações; isto é, perturbações pequenas de sistemas gradient-like continuam sendo gradient-like. Como consequência da caracterização dos atratores para este tipo de sistema, estudamos a atração exponencial de atratores. Por fim, estudamos os conceitos de dimensão de Hausdorff e dimensão fractal de atratores e apresentamos alguns resultados sobre esse assunto, e estudamos a construção de uma nova classe de atratores, os atratores exponenciais fractais. 


\section{Abstract}

In this work, we study a generalization of gradient discrete semigroups, the gradientlike semigroups, some of its properties and its invariance under small perturbations; that is, small perturbations of gradient-like semigroups are still gradient-like semigroups. As a consequence of the characterization of the attractors for this sort of semigroups, we study the exponential attraction of attractors. Finally, we study some concepts of Hausdorff dimension and fractal dimension and present some results about this subject, and we studied the construction of a new class of attractors, the exponential fractal attractors. 


\section{Sumário}

Introdução 1

$\begin{array}{lll}1 & \text { Preliminares } & 5\end{array}$

1.1 Resultados básicos de Análise . . . . . . . . . . . . . . . . . . . . . . 5

1.2 Noções básicas e fatos $\ldots \ldots \ldots$. . . . . . . . . . . . . . . . . . . . . . . . 6

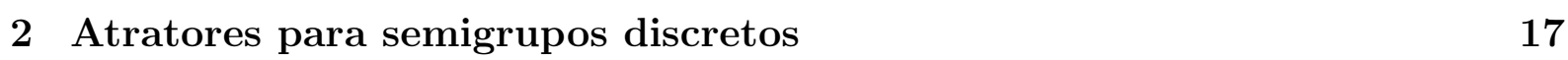

2.1 Existência de atratores para semigrupos discretos . . . . . . . . . . . . . . 17

2.2 Semicontinuidades superior e inferior de atratores $\ldots \ldots \ldots \ldots$

2.3 Semigrupos discretos gradientes . . . . . . . . . . . . . . . . . . . 22

2.4 Semigrupos discretos gradient-like . . . . . . . . . . . . . . . . . 25

2.5 Atratores exponenciais $\ldots \ldots \ldots \ldots$. . . . . . . . . . . . . . . 40

2.6 Taxa de convergência de atratores exponenciais . . . . . . . . . . . . . 44

3 Dimensão fractal e dimensão de Hausdorff de atratores 47

3.1 Dimensão, dimensão de Hausdorff e dimensão fractal $\ldots$. . . . . . . . . . . . 48

3.2 Projeções de conjuntos compactos com dimensão fractal finita . . . . . . . 53

3.3 Dimensão de conjuntos compactos negativamente invariantes . . . . . . . . 57

\begin{tabular}{|lll}
4 & Construção de atratores exponenciais fractais & 71
\end{tabular} 


\section{Introdução}

Em meio aos semigrupos existe a classe dos semigrupos gradientes, que são semigrupos aos quais temos associada uma função de Liapunov. Para estes semigrupos existem resultados que caracterizam os atratores de forma geral; mais especificamente, os atratores para este tipo de sistema são uniões de conjuntos instáveis de pontos de equilíbrio, no caso em que o conjunto dos pontos de equilíbrio é finito. Recordemos que o conjunto instável $W^{u}\left(e^{*}\right)$ de um ponto de equilíbrio $e^{*}$ é o conjunto de pontos do espaço de fase pelos quais existe uma órbita para trás que converge para o ponto de equilíbrio $e^{*}$ (quando o tempo tende para $-\infty)$. Estes resultados estão fortemente firmados sobre a existência da função de Liapunov. O problema que surge quando introduzimos uma certa perturbação a um semigrupo gradiente é que podemos perder a função de Liapunov no problema perturbado.

Assim, este trabalho tem como objetivo introduzir uma noção que estenda o conceito de semigrupos discretos gradientes, os semigrupos discretos gradient-like, de maneira que esta noção preserve a propriedade da caracterização dos atratores; isto é, os atratores para este tipo de problema continuem com a estrutura gradiente, mas de tal forma que este novo conceito seja estável por pequenas perturbações.

Esta construção deixa para trás a função de Liapunov propriamente dita e estuda quais são as estruturas dinâmicas que ela introduz nos atratores, que são:

- as órbitas, tanto para frente quanto para trás, convergem para pontos de equilíbrio;

- não existem contornos fechados formados por órbitas no atrator.

Ao olharmos somente estas propriedades, podemos definir o conceito de semigrupo gradient-like à partir delas e verificar que elas são estáveis por meio de perturbações. Os 
atratores dos semigrupos nesta classe possuem, como consequência de sua estrutura, outras propriedades dinâmicas bastante interessantes. Entre essas propriedades destacamos a atração exponencial, que explicamos a seguir.

Atratores exponenciais Com a caracterização do atrator através das variedades instáveis $W^{u}\left(e_{i}^{*}\right)$, de pontos de equilíbrio $e_{i}^{*}, 1 \leq i \leq n$, podemos estudar como estas variedades instáveis atraem as órbitas em sua vizinhança. Suponhamos que tenhamos uma atração com taxa exponencial; isto é, para alguma vizinhança pequena $V$ de $e_{i}^{*}$

$$
\operatorname{dist}\left(T^{n}\left(u_{0}\right), W^{u}\left(e_{i}^{*}\right) \cap V\right) \leq c e^{-k_{i} n},
$$

durante o tempo no qual a órbita $T^{n}\left(u_{0}\right)$ permanece em $V$. Podemos então concluir que conjuntos limitados são atraídos exponencialmente pelo atrator $\mathcal{A}$; isto é, se $B$ é um conjunto limitado, então

$$
\operatorname{dist}\left(T^{n}(B), \mathcal{A}\right) \leq C e^{-\omega n},
$$

onde $\omega$ é uma constante positiva e $C$ é uma constante positiva que depende de B. Mais ainda, se tivermos uma família de atratores a um parâmetro $\eta$ e a atração exponencial discutida acima for uniforme com relação a este parâmetro temos então a atração destes atratores; ou seja,

$$
\operatorname{dist}\left(\mathrm{A}_{\eta}, \mathrm{A}_{0}\right) \leq \mathrm{L} \epsilon(\eta)^{\mathrm{r}}
$$

onde $L$ e $r$ são constantes positivas e $\epsilon(\eta)$ mede a proximidade entre os semigrupos $\left\{T_{\eta}^{n}: n \in \mathbb{N}\right\}$ e $\left\{T_{0}^{n}: n \in \mathbb{N}\right\}$.

Não é difícil ver que para um atrator num semigrupo discreto gradient-like, a dimensão de Hausdorff do atrator $\mathcal{A}$ é o máximo das dimensões dos conjuntos instáveis $W^{u}\left(e_{i}^{*}\right)$, onde $\mathcal{E}=\left\{e_{1}^{*}, \ldots, e_{k}^{*}\right\}$ é o conjunto dos pontos de equilíbrio do semigrupo. Sabemos ainda que se um espaço métrico compacto tem dimensão topológica finita $m$ então ele pode ser imerso em $\mathbb{R}^{2 m+1}$ (vide Munkres [18]). Assim gostaríamos de saber, em vista deste resultado, se o mesmo é valido para conjuntos compactos com dimensão de Hausdorff finita. Infelizmente, este não é o caso. Para isto precisamos introduzir o conceito de dimensão fractal, e devido a um resultado de Mañé [17 podemos projetar conjuntos compactos com dimensão fractal finita $n$, de maneira injetiva, em um espaço vetorial de dimensão finita maior que $2 n+1$. Por fim, construímos os chamados atratores exponenciais fractais, que são uma extensão do conceito de atratores exponenciais para semigrupos gerais (não 
necessariamente gradient-like). Sabemos que nem todo atrator para um semigrupo tem a característica de atrair exponencialmente as órbitas, mas podemos pedir um pouco menos na definição da atrator (no nosso caso, excluir a invariância e pedir somente a invariância positiva) a fim de tornar viável a atração exponencial. Mas para que este atrator continue com boas propriedades, por exemplo poder ser projetado de forma injetiva num espaço de dimensão finita, pedimos também para que este objeto tenha dimensão fractal finita. Os resultados que apresentaremos neste trabalho são uma versão corrigida e simplificada dos resultados contidos no primeiro capítulo de Eden-Foias-Nicolaenko-Temam [9].

Este trabalho tem como principal objetivo estudar e generalizar o conteúdo do Capítulo 2 de Hale [12]. Em particular, os resultados sobre dimensão fractal apresentados nesta referência são corrigidos e melhorados seguindo Carvalho-Langa-Robinson [6]. Os resultados relacionados a sistemas discretos gradientes não se encontram nesta referência, bem como os resultados relacionados a continuidade de atratores usando a atração uniforme. Para este último, os análogos no caso contínuo podem ser encontrados em Babin-Vishik [1]. Os resultados sobre gradient-like têm sua versão análoga para o caso contínuo no artigo de Carvalho-Langa [4]. 


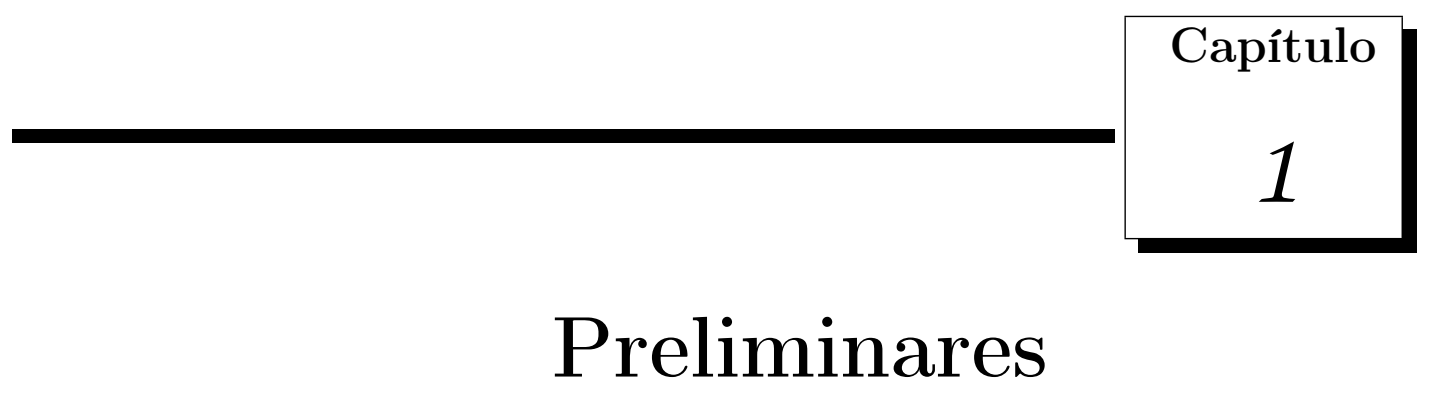

\subsection{Resultados básicos de Análise}

Nesta seção pretendemos abordar alguns resultados básicos de espaços métricos e análise funcional relacionados aos espaços de Banach, que serão bastante usados. Vamos admitir que o leitor tenha um conhecimento básico nesta área, como a definição e algumas propriedades básicas de espaços de Banach e operadores lineares limitados. Os resultados serão somente enunciados, sendo omitidas as suas demonstrações, podendo estas ser encontradas em Brezis [3] e Rudin [20].

Teorema 1.1.1 (Princípio da Contração de Banach). Seja X um espaço métrico completo, $d: X \times X \rightarrow[0, \infty)$ sua métrica e $T: X \rightarrow X$ uma função tal que

$$
d(T(x), T(y)) \leqslant C d(x, y), \quad \forall x, y \in X
$$

para algum $0 \leqslant C<1$. Então $T$ possui um único ponto fixo $x^{*}$ em $X$; isto é, existe um único $x^{*}$ em $X$ tal que $T x^{*}=x^{*}$.

Teorema 1.1.2. Seja $X$ um espaço métrico completo. Um subconjunto $A \subset X$ é relativamente compacto se, e somente se, para cada $\epsilon>0$ existe um subconjunto relativamente compacto $A_{\epsilon} \subset X$ tal que $A \subset \mathcal{O}_{\epsilon}\left(A_{\epsilon}\right)$, onde $\mathcal{O}_{\epsilon}\left(A_{\epsilon}\right)$ é a vizinhança de raio $\epsilon$ de $A_{\epsilon}$. 
Teorema 1.1.3 (Teorema do Gráfico Fechado). Sejam X, Y espaços de Banach e T : $X \rightarrow Y$ uma aplicação linear. Então $T$ é limitada se, e somente se $G(T)=\{(x, T x)$ : $x \in X\} \subset X \times Y$ é fechado em $X \times Y$ com a topologia produto.

\subsection{Noções básicas e fatos}

Nesta seção introduzimos a noção de atratores para semigrupos discretos dissipativos não-lineares. Nosso objetivo é apresentar uma coleção auto-suficiente de resultados sobre o assunto, que são necessários para o estudo de atratores para semigrupos discretos e suas propriedades. Todas as definições e resultados serão feitos visando o resultado principal desta seção, que caracteriza os semigrupos discretos que têm atratores globais.

Seja $X$ um espaço métrico e $d: X \times X \rightarrow[0, \infty)$ sua métrica. Denote por $\mathcal{C}(X)$ o conjunto dos operadores contínuos de $X$ em $X$, por $\mathbb{N}=\{0,1,2, \cdots\}$ o conjunto dos números naturais, por $\mathbb{N}^{*}=\mathbb{N} \backslash\{0\}$ o conjunto dos números naturais sem o zero, por $\mathbb{Z}=\{0, \pm 1, \pm 2, \cdots\}$ o conjunto dos números inteiros, por $\mathbb{Z}^{-}=\{0,-1,-2,-3, \cdots\}$ o conjunto dos números inteiros não-positivos e por $\mathbb{Z}_{n}^{-}=n+\mathbb{Z}^{-}\left(\mathbb{Z}_{n}^{+}=n+\mathbb{N}\right)$ o conjuntos dos números inteiros que são menores (maiores) ou iguais à $n, n \in \mathbb{Z}$. Se $T \in \mathcal{C}(X)$, a família de operadores $\left\{T^{n}: n \in \mathbb{N}\right\} \subset \mathcal{C}(X)$ será chamada um semigrupo discreto.

Para um dado semigrupo $\left\{T^{n}: n \in \mathbb{N}\right\}$, um ponto $x \in X$ e um subconjunto $B \subset X$, definimos:

- Para cada $n \in \mathbb{N}$, a imagem de $B$ sobre $T^{n}$,

$$
T^{n}(B):=\left\{T^{n}(x): x \in B\right\}
$$

- A órbita positiva de $B$,

$$
\gamma^{+}(B):=\bigcup_{n \in \mathbb{N}} T^{n}(B)
$$

- A órbita parcial entre dois números naturais $n$ e $n^{\prime}$,

$$
\gamma_{\left[n, n^{\prime}\right]}^{+}(B):=\bigcup_{n \leqslant k \leqslant n^{\prime}} T^{k}(B)
$$


- A órbita de $T^{n}(B)$,

$$
\gamma_{n}^{+}(B):=\bigcup_{k \in \mathbb{N}} T^{k+n}(B)
$$

- A função $\mathbb{N} \ni n \mapsto T^{n}(x) \in X$ é a solução através de $x$ do problema discreto de valor inicial

$$
\begin{aligned}
& x_{n+1}=T\left(x_{n}\right), n \in \mathbb{N}, \\
& x_{0}=x .
\end{aligned}
$$

Definição 1.2.1. Um semigrupo discreto $\left\{T^{n}: n \in \mathbb{N}\right\}$ é dito limitado se $\gamma^{+}(B)$ é limitado sempre que $B$ é um subconjunto limitado de $X$.

Observação 1.2.2. Os operadores $T \in \mathcal{C}(X)$ não precisam ser limitados em subconjuntos limitados de $X$, uma vez que subconjuntos limitados de $X$ não são necessariamente relativamente compactos. Se assumirmos que T é limitado em subconjuntos limitados de $X$, então $\gamma_{\left[n, n^{\prime}\right]}^{+}(B)$ é limitado para cada subconjunto limitado $B$ de $X$ e $n, n^{\prime} \in \mathbb{N}$. Ainda assim, o semigrupo $\left\{T^{n}: n \in \mathbb{N}\right\}$ não é necessariamente limitado.

Agora definimos solução para trás através de um ponto $x \in X$ e órbita para trás de um subconjunto $B$ de $X$.

Definição 1.2.3. Uma solução para trás através de $x \in X$ é uma função $\phi: \mathbb{Z}^{-} \rightarrow X$ tal que $\phi(0)=x$ e, para cada $k \in \mathbb{Z}^{-}, T^{n}(\phi(k))=\phi(n+k)$ para $0 \leqslant n \leqslant-k$. Uma solução global através de $x \in X$ é uma função $\phi: \mathbb{Z} \rightarrow X$ tal que $\phi(0)=x$ e, para cada $k \in \mathbb{Z}$, $T^{n}(\phi(k))=\phi(n+k)$, para todo $n \in \mathbb{N}$. Como $T$ não é necessariamente injetiva, se uma solução para trás existe ela não precisa ser única. Se existe uma solução para trás através de $x$, podemos definir a órbita negativa de $x \in X$ como

$$
\gamma^{-}(x)=\bigcup_{n \in \mathbb{N}} H(n, x)
$$

onde

$H(n, x)=\left\{y \in X:\right.$ existe uma solução para trás $\phi: \mathbb{Z}^{-} \rightarrow X$ através de $x$ com $\left.\phi(-n)=y\right\}$.

$A$ órbita global através de $x$ (se ela existe) é definida como $\gamma(x):=\gamma^{+}(x) \cup \gamma^{-}(x)$. 
Se $B \subset X$ é tal que existe uma solução para trás através de cada ponto $x \in B$, a órbita negativa de $B$ é definida por $\gamma^{-}(B):=\bigcup_{x \in B} \gamma^{-}(x)$ e a órbita global de $B$ é definida por $\gamma(B):=\bigcup_{x \in B} \gamma(x)$.

Podemos definir os conjuntos $\omega$-limite e $\alpha$-limite como segue

$$
\begin{array}{ll}
\omega(x)=\bigcap_{n \in \mathbb{N}} \overline{\gamma_{n}^{+}(x)}, \quad \omega(B)=\bigcap_{n \in \mathbb{N}} \overline{\gamma_{n}^{+}(B)}, \\
\alpha(x)=\bigcap_{n \in \mathbb{N}} \overline{\gamma_{n}^{-}(x)}, \quad \alpha(B)=\bigcap_{n \in \mathbb{N}} \overline{\gamma_{n}^{-}(B)},
\end{array}
$$

onde se $D \subset X, \bar{D}$ denota o fecho do conjunto $D$ em $X$.

A seguinte caracterização do conjunto $\omega$-limite será frequentemente usada

Proposição 1.2.4. Para cada subconjunto $B$ de $X$, temos

$$
\begin{aligned}
\omega(B)= & \left\{y \in X: \text { existem sequências }\left\{k_{n}\right\}_{n \in \mathbb{N}} \text { em } \mathbb{N}\right. \\
& \left.e\left\{x_{n}\right\}_{n \in \mathbb{N}} \text { em } B \text {, tal que } k_{n} \stackrel{n \rightarrow \infty}{\longrightarrow} \infty \text { e } y=\lim _{n \rightarrow+\infty} T^{k_{n}}\left(x_{n}\right)\right\} .
\end{aligned}
$$

Demonstração: Primeiramente, seja $y \in \omega(B)$. Então $y \in \cap_{n \in \mathbb{N}} \overline{\gamma_{n}^{+}(B)}$, e assim $y \in$ $\overline{\gamma_{n}^{+}(B)}$, para todo $n \in \mathbb{N}$; isto é, para cada $n \in \mathbb{N}$ existe uma sequência $\left\{y_{k}^{n}\right\}_{k \in \mathbb{N}} \subset \gamma_{n}^{+}(B)$ tal que $y_{k}^{n} \stackrel{k \rightarrow \infty}{\longrightarrow} y$, para todo $n \in \mathbb{N}$. Mas $y_{k}^{n} \in \gamma_{n}^{+}(B)$ para todo $n \in \mathbb{N}$ e $k \in \mathbb{N}$, e logo existem $\left\{x_{k}^{n}\right\}_{n, k \in \mathbb{N}} \subset B$ e $\left\{q_{k}^{n}\right\}_{n, k \in \mathbb{N}} \subset \mathbb{N}$ tais que

$$
y_{k}^{n}=T^{n+q_{k}^{n}}\left(x_{k}^{n}\right)
$$

Sabemos que dados $n \in \mathbb{N}$ e $\epsilon>0$, existe $k_{(n, \epsilon)} \in \mathbb{N}$ tal que

$$
\left\|y_{k}^{n}-y\right\|<\epsilon, \text { se } k \geq k_{(n, \epsilon)}
$$

isto é, $\left\|T^{n+q_{k}^{n}}\left(x_{k}^{n}\right)-y\right\|<\epsilon$ se $k \geq k_{(n, \epsilon)}$. Defina então $k_{n}:=n+q_{k_{\left(n, \frac{1}{n}\right)}^{n}}$ e $x_{n}:=x_{k_{\left(n, \frac{1}{n}\right)}^{n}}$, assim

$$
\left\|T^{k_{n}} x_{n}-y\right\|<\frac{1}{n} \longrightarrow 0, \quad n \rightarrow \infty .
$$

Portanto $y=\lim _{n \rightarrow \infty} T^{k_{n}} x_{n}$. Para a recíproca, seja $y \in X$ tal que existam sequências $\left\{k_{n}\right\}_{n \in \mathbb{N}} \subset \mathbb{N}$ e $\left\{x_{n}\right\}_{n \in \mathbb{N}} \subset B$ tais que $y=\lim _{n \rightarrow \infty} T^{k_{n}} x_{n}$. Assim, fixado $p \in \mathbb{N}$ temos 
$\left\{T^{k_{n}}\left(x_{n}\right)\right\}_{k_{n} \geq p} \subset \gamma_{p}^{+}(B)$, assim $y \in \overline{\gamma_{p}^{+}(B)}$, para todo $p \in \mathbb{N}$, portanto $y \in \omega(B)$.

A seguir definimos as noções de atração, absorção e invariância sobre o semigrupo discreto $\left\{T^{n}: n \in \mathbb{N}\right\}$. Para este fim, relembremos as definições da semi-distância de Hausdorff $\operatorname{dist}_{H}(A, B)$ entre dois subconjuntos $A$ e $B$ de $X$

$$
\operatorname{dist}_{H}(A, B):=\sup _{x \in A} \inf _{y \in B} d(x, y)
$$

Denotaremos por $\operatorname{dist}(A, B)$ a distância usual entre conjuntos; isto é,

$$
\operatorname{dist}(A, B):=\inf _{x \in A} \inf _{y \in B} d(x, y)
$$

Relembremos também a definição da medida de não-compacidade de Kuratowski para conjuntos limitados segundo Deimling [8]

$$
\begin{array}{r}
\beta(B)=\inf \{d>0: B \text { admite cobertura finita por conjuntos } \\
\text { de diâmetro menor ou igual a } d\} ;
\end{array}
$$

que tem como principais propriedades:

(a) $\beta(B)=0$ se e somente se $\bar{B}$ é compacto,

(b) $\beta$ é uma seminorma; isto é, $\beta(\lambda B)=|\lambda| \beta(B)$ e $\beta\left(B_{1}+B_{2}\right) \leq \beta\left(B_{1}\right)+\beta\left(B_{2}\right)$,

(c) se $B_{1} \subset B_{2}$ então $\beta\left(B_{1}\right) \leq \beta\left(B_{2}\right)$ e $\beta\left(B_{1} \cup B_{2}\right)=\max \left\{\beta\left(B_{1}\right), \beta\left(B_{2}\right)\right\}$

Definição 1.2.5. Sejam $A$ e $B$ subconjuntos de um espaço métrico $X$. Dizemos que $A$ atrai $B$ sobre o semigrupo discreto $\left\{T^{n}: n \in \mathbb{N}\right\}$ se

$$
\lim _{n \rightarrow \infty} \operatorname{dist}_{H}\left(T^{n}(B), A\right)=0
$$

Se existe um $n_{0} \in \mathbb{N}$ tal que $T^{n}(B) \subset A$ para todo $n \geqslant n_{0}$, dizemos que $A$ absorve $B$. Em particular, se $A$ absorve $B$, então $A$ atrai $B$ (a recíproca não é verdadeira). Dizemos que um subconjunto $A$ de $X$ é invariante (ou positivamente invariante ou negativamente invariante) com respeito ao semigrupo discreto $\left\{T^{n}: n \in \mathbb{N}\right\}$ se $T(A)=A($ ou $T(A) \subset A$ ou $T(A) \supset A)$. Um ponto fixo $x^{*}$ para $T$; isto é, um ponto $x^{*} \in X$ tal que $T\left(x^{*}\right)=x^{*} e ́$ 
invariante sobre $\left\{T^{n}: n \in \mathbb{N}\right\}$. Um ponto fixo para a aplicação $T$ será também chamado um ponto de equilíbrio ou um equilíbrio para o semigrupo discreto $\left\{T^{n}: n \in \mathbb{N}\right\} e$ $\mathbb{Z} \ni n \mapsto x^{*} \in X$ é uma solução estacionária ou uma solução de equilíbrio para o semigrupo discreto $\left\{T^{n}: n \in \mathbb{N}\right\}$.

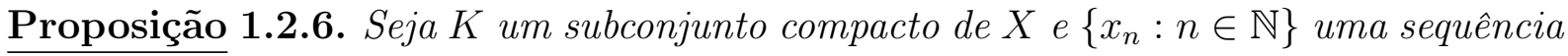
em $X$ tal que

$$
\operatorname{dist}\left(x_{n}, K\right) \stackrel{n \rightarrow \infty}{\longrightarrow} 0
$$

então $\left\{x_{n}: n \in \mathbb{N}\right\}$ tem uma subsequência convergente. Se $K$ atrai um conjunto compacto $K_{1}$, então $\gamma^{+}\left(K_{1}\right)$ é relativamente compacto $e \emptyset \neq \omega\left(K_{1}\right) \subset K$.

Demonstração Para a primeira parte, notemos que dado $m \in \mathbb{N}$, existem $n_{m} \in \mathbb{N}$ e $y_{n_{m}} \in$ $K$ tais que $\operatorname{dist}\left(x_{n_{m}}, K\right)<\frac{1}{2 m}$ e $d\left(x_{n_{m}}, y_{n_{m}}\right)<\operatorname{dist}\left(x_{n_{m}}, K\right)+\frac{1}{2 m}<\frac{1}{m}$. Como $\left\{y_{n_{m}}\right\}_{m \in \mathbb{N}}$ é uma sequência em $K$, que é compacto, $y_{n_{m}} \stackrel{m \rightarrow \infty}{\longrightarrow} y_{0} \in K$, a menos de subsequências. Assim, obtemos

$$
d\left(x_{n_{m}}, y_{0}\right) \leq d\left(x_{n_{m}}, y_{n_{m}}\right)+d\left(y_{n_{m}}, y_{0}\right) \longrightarrow 0, \text { quando } m \rightarrow \infty
$$

isto é, $\left\{x_{n}\right\}_{n \in \mathbb{N}}$ possui uma subsequência convergente. Agora, para a segunda parte, dado $\epsilon>0$ existe $n_{0} \in \mathbb{N}$ tal que

$$
T^{n}\left(K_{1}\right) \subset \mathcal{O}_{\frac{\epsilon}{2}}(K), \text { para todo } n \geq n_{0},
$$

onde $\mathcal{O}_{\epsilon}(K)$ denota a $\epsilon$-vizinhança do conjunto $K$. Assim, $\cup_{n \geq n_{0}} T^{n}\left(K_{1}\right)$ tem medida de não-compacidade menor ou igual à $\epsilon$. Segue facilmente do fato que $\cup_{n=1}^{n_{0}-1} T^{n}\left(K_{1}\right)$ é compacto, que a medida de não-compacidade de $\gamma^{+}\left(K_{1}\right)$ é nula, o que nos dá $\gamma^{+}\left(K_{1}\right)$ relativamente compacto.

Finalmente, temos $\overline{\gamma_{n}^{+}\left(K_{1}\right)}$ compacto e não-vazio, para todo $n \in \mathbb{N}$ e $\overline{\gamma_{n}^{+}\left(K_{1}\right)} \subset \overline{\gamma_{m}^{+}\left(K_{1}\right)}$ para $m \leq n$, ou seja, a família $\left\{\overline{\gamma_{n}^{+}\left(K_{1}\right)}\right\}_{n \in \mathbb{N}}$ possui a propriedade da interseção finita e assim

$$
\omega\left(K_{1}\right)=\cap_{n \in \mathbb{N}} \overline{\gamma_{n}^{+}\left(K_{1}\right)} \neq \emptyset .
$$

Dados $y \in \omega\left(K_{1}\right)$ e $\epsilon>0$, existe $n_{0} \in \mathbb{N}$ tal que

$$
y \in \overline{\gamma_{n_{0}}^{+}\left(K_{1}\right)} \subset \mathcal{O}_{\epsilon}(K),
$$


$\operatorname{assim} \operatorname{dist}(y, K) \leq \epsilon$ e como $\epsilon$ é arbitrário, segue o resultado.

Lema 1.2.7. Se $B \subset X$, então $T(\omega(B)) \subset \omega(B)$. Se $B$ é tal que $\omega(B)$ é compacto $e$ atrai $B$, então $\omega(B)$ é invariante. Ainda mais, se $\omega(B)$ atrai um conjunto conexo $C$ que contém $\omega(B)$, então $\omega(B)$ é conexo. Similarmente, $T(\alpha(B)) \subset \alpha(B)$ e se $\alpha(B)$ é compacto $\lim _{n \rightarrow \infty} \operatorname{dist}_{H}(H(n, B), \alpha(B)) \rightarrow 0$, então $\alpha(B)$ é invariante.

Demonstração: Se $\omega(B)=\emptyset$, não há nada a mostrar. Se $\omega(B) \neq \emptyset$, obtemos da continuidade de $T$ que se $y \in \omega(B)$ então existem sequências $\left\{k_{n}\right\}_{n \in \mathbb{N}} \subset \mathbb{N}$ e $\left\{x_{n}\right\}_{n \in \mathbb{N}} \subset$ $B$ tais que $y=\lim _{n \rightarrow \infty} T^{k_{n}} x_{n}$ e $\operatorname{assim} T y=T\left(\lim _{n \rightarrow \infty} T^{k_{n}} x_{n}\right)=\lim _{n \rightarrow \infty} T\left(T^{k_{n}} x_{n}\right)=$ $\lim _{n \rightarrow \infty} T^{k_{n}+1} x_{n} \in \omega(B)$ e portanto $T(\omega(B)) \subset \omega(B)$.

Nos resta mostrar que, se $\omega(B)$ é compacto e atrai $B$, então $\omega(B) \subset T(\omega(B))$. Para $x \in \omega(B)$, existem sequências $k_{n} \rightarrow \infty$ e $x_{n} \in B$ tais que $T^{k_{n}}\left(x_{n}\right) \rightarrow x$ quando $n \rightarrow \infty$. Uma vez que $k_{n} \rightarrow \infty$, existe $n_{0} \in \mathbb{N}$ tal que $k_{n}>1$ para todo $n \geqslant n_{0}$. Portanto $T\left(T^{k_{n}-1}\left(x_{n}\right)\right)=T^{k_{n}}\left(x_{n}\right) \rightarrow x$ quando $n \rightarrow \infty$. Como $\omega(B)$ é compacto e atrai $B$ temos $\operatorname{dist}\left(T^{k_{n}-1}\left(x_{n}\right), \omega(B)\right) \stackrel{n \rightarrow \infty}{\longrightarrow} 0$. Segue da Proposição 1.2 .6 que $\left\{T^{k_{n}-1}\left(x_{n}\right)\right\}_{n \in \mathbb{N}}$ tem uma subsequência convergente (que denotaremos novamente por $\left\{T^{k_{n}-1}\left(x_{n}\right)\right\}_{n \in \mathbb{N}}$ ). Se $T^{k_{n}-1}\left(x_{n}\right) \rightarrow y \in \omega(B)$, então $T y=x$. Portanto, $\omega(B)=T(\omega(B))$.

Agora provemos a afirmação sobre a conexidade de $\omega(B)$. Suponha que $\omega(B)$ é desconexa, então $\omega(B)$ é a união disjunta de dois conjuntos compactos (portanto separados por uma distância positiva), mas $\omega(B)$ atrai $C$, $\log _{0} \operatorname{dist}_{H}\left(T^{n}(C), \omega(B)\right) \rightarrow 0$, mas isto implica que $T^{n}(C)$ deve estar contido em ambas as componentes de $\omega(B)$ para $n$ suficientemente grande, e isto é uma contradição com o fato de que $T^{n}(C)$ é conexo.

A demonstração para o caso $\alpha(B)$ é completamente análoga.

Observação 1.2.8. Segue imediatamente da primeira parte do Lema 1.2.7 que, se $x \in X$ e $\omega(x)=\left\{x^{*}\right\}$, então $x^{*}$ é um equlíbrio (ponto fixo de $T$ ). Um resultado similar se verifica para $\alpha(x)$ (também para $\alpha_{\phi}(x)$ definido a seguir no Lema 1.2.10).

Lema 1.2.9. Se $B$ é um subconjunto não-vazio de $X$ tal que $\overline{\gamma_{n_{0}}^{+}(B)}$ é compacto, para algum $n_{0} \in \mathbb{N}$, então $\omega(B)$ é não-vazio, compacto, invariante e $\omega(B)$ atrai $B$.

Demonstração: Como $\overline{\gamma_{n}^{+}(B)}$ é não-vazio e compacto, $n \geqslant n_{0}$, temos que $\omega(B)$ é nãovazio e compacto, uma vez que uma família de conjuntos compactos com a propriedade da interseção finita tem interseção compacta e não-vazia. 
Mostremos agora que $\omega(B)$ atrai $B$. Suponha que não, então existem $\epsilon_{0}>0$ e sequên$\operatorname{cias}\left\{x_{n}: n \in \mathbb{N}\right\}$ em $B,\left\{k_{n}: n \in \mathbb{N}\right\}$ em $\mathbb{N}$ com $k_{n} \stackrel{n \rightarrow \infty}{\longrightarrow} \infty$, tais que $d\left(T^{k_{n}}\left(x_{n}\right), \omega(B)\right)>\epsilon_{0}$ para todo $n \in \mathbb{N}$. Como $\overline{\gamma_{n_{0}}^{+}(B)}$ é compacto e $\left\{T^{k_{n}}\left(x_{n}\right), n \geqslant n_{1}\right\} \subset \overline{\gamma_{n_{0}}^{+}(B)}$ para algum $n_{1} \in \mathbb{N}$, existem subsequências $k_{n_{j}} \stackrel{j \rightarrow \infty}{\longrightarrow} \infty$ e $x_{n_{j}} \in B$ tais que $T^{k_{n_{j}}}\left(x_{n_{j}}\right)$ converge para $y \in \omega(B)$. Isto nos leva a uma contradição e mostra que $\omega(B)$ atrai $B$.

Segue agora do Lema 1.2 .7 que $\omega(B)$ é invariante e a prova está completa.

Lema 1.2.10. Suponha que $x \in X$ é tal que existe uma solução para trás $\phi: \mathbb{Z}^{-} \rightarrow X$ através de $x$ e tal que $\overline{\phi\left(\mathbb{Z}^{-}\right)}$é compacto. Defina

$$
\alpha_{\phi}(x)=\left\{v \in X: \exists k_{n} \rightarrow \infty \text { tal que } \phi\left(-k_{n}\right) \rightarrow v\right\}
$$

Então, $\alpha_{\phi}(x)$ é não-vazio, compacto e invariante.

Demonstração: Claramente podemos ver que $\alpha_{\phi}(x)=\cap_{n \in \mathbb{N}} \overline{\phi\left(\mathbb{Z}_{-n}^{-}\right)}$e disto segue imediatamente que $\alpha_{\phi}(x)$ é não-vazio e compacto.

Falta ainda provar que $\alpha_{\phi}(x)$ é invariante. De fato, se $y \in \alpha_{\phi}(x)$, então existe uma sequência $k_{n} \stackrel{n \rightarrow \infty}{\longrightarrow}+\infty$ tal que $\phi\left(-k_{n}\right) \stackrel{n \rightarrow \infty}{\longrightarrow} y$. Da continuidade de $T: X \rightarrow X$ obtemos que $T\left(\phi\left(-k_{n}\right)\right)=\phi\left(-k_{n}+1\right) \stackrel{n \rightarrow \infty}{\longrightarrow} T(y)$ e portanto $T(y) \in \alpha_{\phi}(x)$. Por outro lado, se $w \in$ $\alpha_{\phi}(x)$, existe uma sequência $k_{n} \stackrel{n \rightarrow \infty}{\longrightarrow} \infty$ (assuma que $k_{n} \geqslant 1, n \in \mathbb{N}$ ) tal que $\phi\left(-k_{n}\right) \stackrel{n \rightarrow \infty}{\longrightarrow}$ $w$. Como $\left\{\phi\left(-k_{n}-k\right): k \in \mathbb{N}\right\}$ é relativamente compacto, tomando subsequências se necessário, existe $z \in X$ tal que $\phi\left(-k_{n}-k\right) \stackrel{n \rightarrow \infty}{\longrightarrow} z \mathrm{e} z \in \alpha_{\phi}(x)$. Segue da unicidade do limite que $T^{k}(z)=w$.

Outro conceito importante é

Definição 1.2.11. Um semigrupo discreto $\left\{T^{n}: n \in \mathbb{N}\right\}$ é dito assintoticamente compacto se, para qualquer subconjunto fechado, limitado e não-vazio $B \subset X$, para o qual $T(B) \subset B$, existe um conjunto compacto $J \subset B$ que atrai $B$.

Proposição 1.2.12. Um semigrupo discreto $\left\{T^{n}: n \in \mathbb{N}\right\}$ é assintoticamente compacto se $\left\{T^{k_{n}} x_{n}: n \in \mathbb{N}\right\}$ é relativamente compacto sempre que $\left\{T^{k_{n}} x_{n}: n \in \mathbb{N}\right\}$ é limitado, $\left\{x_{n}: n \in \mathbb{N}\right\}$ é limitado em $X$ e $k_{n} \stackrel{n \rightarrow \infty}{\longrightarrow} \infty$.

Demonstração: Seja $B \subset X$ um conjunto fechado, limitado e não-vazio tal que $T(B) \subset$ $B$. Então, segue do Lema 1.2 .9 que $\omega(B) \subset B$ é não-vazio, compacto, invariante e atrai $B$, pois as hipóteses nos garantem que $\overline{\gamma_{n_{0}}^{+}(B)}$ é compacto, para qualquer $n_{0} \in \mathbb{N}$. 
Lema 1.2.13. Se $\left\{T^{n}: n \in \mathbb{N}\right\}$ é um semigrupo discreto assintoticamente compacto e $B$ é um subconjunto não-vazio de $X$ tal que $\gamma_{n_{0}}^{+}(B)$ é limitado, para algum $n_{0} \in \mathbb{N}$, então $\omega(B)$ é não-vazio, compacto, invariante e $\omega(B)$ atrai $B$.

Demonstração: Como $T\left(\gamma_{n_{0}}^{+}(B)\right) \subset \gamma_{n_{0}}^{+}(B)$, segue da continuidade de $T^{k}: X \rightarrow X$ que $\omega(B) \subset T^{k}\left(\overline{\gamma_{n_{0}}^{+}(B)}\right) \subset \overline{\gamma_{n_{0}}^{+}(B)}$. Como $\left\{T^{n}: n \in \mathbb{N}\right\}$ é assintoticamente compacto temos que existe um compacto $J \subset \overline{\gamma_{n_{0}}^{+}(B)}$ que atrai $\gamma_{n_{0}}^{+}(B)$. Logo, existem sequências $\epsilon_{n} \rightarrow 0$ e $k_{n} \stackrel{n \rightarrow \infty}{\longrightarrow} \infty$ tais que $T^{k}\left(\overline{\gamma_{n_{0}}^{+}(B)}\right) \subset \mathcal{O}_{\epsilon_{n}}(J)$ para todo $k \geqslant k_{n}$. Assim, $\omega(B) \subset J$. Como $\omega(B)$ é fechado e $J$ é compacto, temos que $\omega(B)$ é compacto.

Nos resta mostrar que $\omega(B)$ atrai $B$. Se não, existem $\epsilon_{0}>0$ e sequências $x_{n} \in B$ e $k_{n} \stackrel{n \rightarrow \infty}{\longrightarrow} \infty$ tais que $d\left(T^{k_{n}}\left(x_{n}\right), \omega(B)\right)>\epsilon_{0}$. Da compacidade de $J$ e da Proposição 1.2.6. existem sequências $x_{n_{j}} \in B, k_{n_{j}} \stackrel{j \rightarrow \infty}{\longrightarrow} \infty$ e $z \in J$ tais que $T^{k_{n_{j}}}\left(x_{n_{j}}\right) \stackrel{j \rightarrow \infty}{\longrightarrow} z \in \omega(B)$. Isto nos leva à uma contradição $\omega(B)$ atrai $B$. Portanto, $\omega(B)$ é não-vazio, compacto e atrai $B$ e do Lema 1.2.7, segue a invariância.

Definição 1.2.14. Um semigrupo discreto $\left\{T^{n}: n \in \mathbb{N}\right\}$ é dito condicionalmente eventualmente compacto, se para cada conjunto limitado $B \subset X$ tal que $T^{n}(B)$ é limitado para algum $n \in \mathbb{N}$, nós temos que $\overline{T^{n}(B)}$ é compacto. Um semigrupo discreto $\left\{T^{n}: n \in \mathbb{N}\right\}$ é dito eventualmente compacto se é condicionalmente eventualmente compacto e para cada conjunto limitado $B$ em $X$, existe um $n \in \mathbb{N}$ tal que $T^{n}(B)$ é um subconjunto limitado de $X$.

Teorema 1.2.15. Um semigrupo condicionalmente eventualmente compacto é assintoticamente compacto.

Demonstração: Seja $B \subset X$ um conjunto não-vazio, fechado e limitado tal que $T(B) \subset$ $B$. Então, como $\left\{T^{n}: n \in \mathbb{N}\right\}$ é condicionalmente eventualmente compacto temos que $\overline{\gamma_{n}^{+}(B)}$ é compacto para um $n$ suficientemente grande. Assim, do Lema 1.2.9, $\omega(B)=$ $\bigcap_{n \in \mathbb{N}} \overline{\gamma_{n}^{+}(B)} \subset B$ é não-vazio, compacto e atrai $B$. Isto nos mostra que $\left\{T^{n}: n \in \mathbb{N}\right\}$ é assintoticamente compacto.

Definição 1.2.16. Um semigrupo discreto $\left\{T^{n}: n \in \mathbb{N}\right\}$ é dito ponto dissipativo (limitado dissipativo / compacto dissipativo ) se existe um subconjunto limitado $B \subset X$ que atrai pontos (subconjuntos limitados/subconjuntos compactos). 
Observação 1.2.17. Na definição acima podemos trocar a palavra atrai pela palavra absorve sem mudar os significados dos conceitos. De fato, se um subconjunto limitado $B \subset X$ atrai pontos (subconjuntos limitados/subconjuntos compactos), então a vizinhança $\mathcal{O}_{\epsilon}(B)$, com $\epsilon>0$, absorve pontos (subconjuntos limitados/subconjuntos compactos). Claramente, se $B$ absorve pontos (subconjuntos limitados/subconjuntos compactos), então B atrai pontos (subconjuntos limitados/subconjuntos compactos).

Definição 1.2.18. Seja $\left\{T^{n}: n \in \mathbb{N}\right\}$ um semigrupo discreto em um espaço métrico $X$. Um conjunto $\mathcal{A}$ é chamado um atrator global para $\left\{T^{n}: n \in \mathbb{N}\right\}$ se é compacto, invariante e atrai subconjuntos limitados de $X$.

Neste ponto vale notar a unicidade do atrator global para um semigrupo $\left\{T^{n}: n \in \mathbb{N}\right\}$. Sejam $\mathcal{A}$ e $\mathcal{A}^{*}$ dois atratores globais para este semigrupo. Assim

$$
\operatorname{dist}_{H}\left(\mathcal{A}, \mathcal{A}^{*}\right)=\operatorname{dist}_{H}\left(T^{n}(\mathcal{A}), \mathcal{A}^{*}\right) \rightarrow 0 \text {, quando } n \rightarrow \infty,
$$

e assim $\mathcal{A} \subset \mathcal{A}^{*}$. Analogamente $\mathcal{A}^{*} \subset \mathcal{A}$, e temos o resultado.

Observação 1.2.19. Seja $\left\{T^{n}: n \in \mathbb{N}\right\}$ um semigrupo discreto em um espaço métrico $X$. Assuma que $\left\{T^{n}: n \in \mathbb{N}\right\}$ tenha um atrator $\mathcal{A}$. Afirmamos que através de cada ponto $x \in \mathcal{A}$ existe uma solução global limitada $\phi_{x}: \mathbb{Z} \rightarrow X$. De fato, a órbita para frente está sempre bem definida e é limitada; agora, seja $x \in \mathcal{A}=T(\mathcal{A})$, assim existe $x_{-1} \in \mathcal{A}$ tal que $T x_{-1}=x$ e procedendo indutivamente, conseguimos uma sequência $\left\{x_{-n}: n \in \mathbb{N}\right\}$ tal que $x_{0}=x$ e $T x_{-n-1}=x_{-n}$ para todo $n \in \mathbb{N}$, e portanto contruímos uma órbita para trás (lembrando que esta órbita não é unicamente determinada). Defina então

$$
\phi_{x}(j)=\left\{\begin{array}{rl}
T^{j} x, & j \geqslant 0 \\
x_{j}, & j<0
\end{array},\right.
$$

que é uma órbita limitada em $\mathcal{A}$ passando por $x$.

Reciprocamente, cada solução global limitada $\phi: \mathbb{Z} \rightarrow X$ para $\left\{T^{n}: n \in \mathbb{N}\right\}$ é tal que $\phi(\mathbb{Z}) \subset \mathcal{A}$. Tendo dito isto, concluímos que

$$
\mathcal{A}=\{x \in X: \text { existe uma solução global limitada através de } x\}
$$


Lema 1.2.20. Seja $\left\{T^{n}: n \in \mathbb{N}\right\}$ um semigrupo discreto ponto dissipativo e assintoticamente compacto. Assuma que para cada subconjunto compacto $B$ de $X$ existe um $n_{B} \in \mathbb{N}$ tal que $\gamma_{n_{B}}^{+}(B)$ é limitado. Então $\left\{T^{n}: n \in \mathbb{N}\right\}$ é compacto dissipativo.

Demonstração: Como $\left\{T^{n}: n \in \mathbb{N}\right\}$ é ponto dissipativo, existe um conjunto não-vazio, fechado e limitado $B$ que absorve pontos de $X$. Seja $U=\left\{x \in B: \gamma^{+}(x) \subset B\right\}$. Como $B$ absorve pontos, temos que $U$ é não-vazio. Claramente $\gamma^{+}(U) \subset U$ e portanto $U=\gamma^{+}(U)$ é limitado e absorve pontos. Sabemos também que $T\left(\overline{\gamma^{+}(U)}\right) \subset \overline{\gamma^{+}(U)}$ e que $\left\{T^{n}: n \in \mathbb{N}\right\}$ é assintoticamente compacto. Portanto, existe um conjunto compacto $K$, com $K \subset \overline{\gamma^{+}(U)}=\bar{U}$, tal que $K$ atrai $U$ e portanto $K$ atrai pontos de $X$.

$\mathrm{O}$ conjunto $K$ atrai a si mesmo (pois atrai $\bar{U}$ e $K \subset U$ ) e assim (veja a Proposição 1.2.6 $\overline{\gamma^{+}(K)}$ é compacto e $\emptyset \neq \omega(K) \subset K$. O Lema 1.2 .9 implica que $\omega(K)$ é nãovazio, compacto, invariante e atrai $K$. Portanto $\omega(K)$ atrai $K$ que atrai pontos de $X$ e consequentemente $\omega(K)$ atrai pontos de $X$.

Mostremos agora que existe uma vizinhança $V$ de $\omega(K)$ tal que $\gamma_{n}^{+}(V)$ é limitado para algum $n \in \mathbb{N}$. Se esse não é o caso, existem sequências $x_{n} \in X, x_{n} \rightarrow y \in \omega(K)$ e $k_{n} \rightarrow \infty$ tais que $\left\{T^{k_{n}}\left(x_{n}\right): n \in \mathbb{N}\right\}$ não é limitada. Considere $A=\left\{x_{n}: n \in \mathbb{N}\right\}, \operatorname{logo}$ $\bar{A}$ é compacto e $\gamma_{n}^{+}(\bar{A})$ é não-limitada para cada $n \in \mathbb{N}$. Isto contradiz a hipótese de que existe $n_{\bar{A}}$ tal que $\gamma_{n_{\bar{A}}}^{+}(\bar{A})$ é limitado.

Seja $V$ uma vizinhança de $\omega(K)$ e $n_{V} \in \mathbb{N}$ tal que $\gamma_{n_{V}}^{+}(V)$ é limitado. Como $\omega(K)$ atrai pontos de $X$ e $T^{n}$ é contínua, para todo $x \in X$ existem $n_{x}>0$ e uma vizinhança $\mathcal{O}_{x}$ de $x$ tais que $T^{n_{x}}\left(\mathcal{O}_{x}\right) \subset \gamma_{n_{V}}^{+}(V)$. Logo, $T^{k+n_{x}}\left(\mathcal{O}_{x}\right) \subset T^{k}\left(\gamma_{n_{V}}^{+}(V)\right) \subset \gamma_{n_{V}}^{+}(V)$ para todo $k \in \mathbb{N}$; isto é, $T^{n}\left(\mathcal{O}_{x}\right) \subset \gamma_{n_{V}}^{+}(V)$ para todo $n \geqslant n_{x}$, e portanto $\gamma_{n_{V}}^{+}(V)$ absorve uma vizinhança de $x$ para cada $x \in X$. Se $K \subset X$ é um conjunto compacto, para cada $x \in K$ existe $\mathcal{O}_{x}$ como acima. Segue que existem $\left\{x_{i}\right\}_{i=1}^{p} \in K$ tais que $K \subset \cup_{i=1}^{p} \mathcal{O}_{x_{i}}$ e portanto $T^{n}(K) \subset \gamma_{n_{V}}^{+}(V)$, para $n \geqslant \max \left\{n_{x_{i}}: 1 \leqslant i \leqslant p\right\}$, onde $n_{x_{i}}>0$ é dado como acima, para cada $i=1, \ldots, p$. Concluímos assim que $\gamma_{n_{V}}^{+}(V)$ absorve subconjuntos compactos de $X$ e que $\left\{T^{n}: n \in \mathbb{N}\right\}$ é compacto dissipativo.

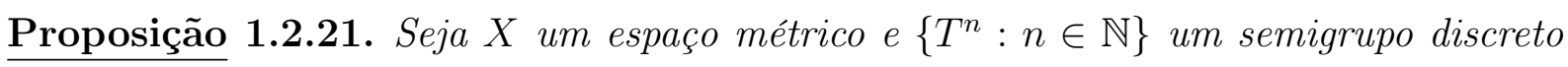
em $X$. Se $K$ é compacto atrai a si mesmo sobre $\left\{T^{n}: n \in \mathbb{N}\right\}$, então $\omega(K)=\cap_{n \in \mathbb{N}} T^{n}(K)$.

Demonstração: Claramente $\cap_{n \in \mathbb{N}} T^{n}(K) \subset \omega(K)$. Agora, para a inclusão contrária, usamos a Proposição 1.2.6 com $K_{1}=K$ para garantir que $\omega(K) \subset K$ e $\gamma^{+}(K)$ é relativamente compacto. Do Lema 1.2 .9 temos que $\omega(K)$ é não-vazio, compacto, invariante e 
atrai $K$. Assim

$$
\omega(K)=T^{n}(\omega(K)) \subset T^{n}(K), \text { para todo } n \in \mathbb{N},
$$

o que prova o nosso resultado.

Definição 1.2.22. Um semigrupo discreto $\left\{T^{n}: n \in \mathbb{N}\right\}$ é dito eventualmente limitado se para cada subconjunto limitado $B$ de $X$ existe $n_{B} \in \mathbb{N}$ tal que $\gamma_{n_{B}}^{+}(B)$ é um subconjunto limitado de $X$.

Finalizamos esta seção com o resultado principal que estávamos buscando, que dá uma caracterização dos semigrupos que possuem atratores globais.

Teorema 1.2.23. Um semigrupo discreto $\left\{T^{n}: n \in \mathbb{N}\right\}$ é eventualmente limitado, ponto dissipativo e assintoticamente compacto se, e somente se, $\left\{T^{n}: n \in \mathbb{N}\right\}$ tem um atrator global $\mathcal{A}$.

Demonstração: Do fato de que $\left\{T^{n}: n \in \mathbb{N}\right\}$ é assintoticamente compacto, ponto dissipativo e eventualmente limitado segue do Lema 1.2.20 que $\left\{T^{n}: n \in \mathbb{N}\right\}$ é compacto dissipativo. Seja $C$ um conjunto limitado que absorve subconjuntos compactos de $X$. Considere $B=\left\{x \in C: \gamma^{+}(x) \subset C\right\}$. Então, $T(\bar{B}) \subset \bar{B}$ e, como $\left\{T^{n}: n \in \mathbb{N}\right\}$ é assintoticamente compacto, existe um conjunto compacto $K \subset \bar{B}$ que atrai $B$ e consequentemente $K$ atrai subconjuntos compactos de $X$.

O conjunto $\mathcal{A}=\omega(K)$ é não-vazio, compacto, invariante e atrai subconjuntos compactos de $X$. Para mostrar que $\mathcal{A}$ é independente da escolha de $K$ suponha que $K_{1}$ é compacto e atrai subconjuntos compactos de $X$, então $\omega\left(K_{1}\right) \subset \omega(K) \subset \omega\left(K_{1}\right)$, pela Proposição 1.2 .6 .

Seja $B$ um subconjunto limitado de $X$, como $\left\{T^{n}: n \in \mathbb{N}\right\}$ é eventualmente limitado e assintoticamente compacto, segue do Lema 1.2 .13 que $\omega(B)$ é não-vazio, compacto, invariante e atrai $B$. Como $\omega(B)$ é compacto e invariante temos que $\omega(B) \subset \mathcal{A}$ e consequentemente $\mathcal{A}$ atrai $B$.

Claramente, se $\left\{T^{n}: n \in \mathbb{N}\right\}$ tem um atrator global, ele é eventualmente limitado, ponto dissipativo e assintoticamente compacto, esta última seguindo diretamente da Proposição 1.2 .12 . 


\section{Capítulo}

2

\section{Atratores para semigrupos discretos}

\subsection{Condições suficientes para a existência de atratores para semigrupos discretos}

Nesta seção apresentaremos alguns resultados que são comumente usados para garantir que um semigrupo discreto é assintoticamente compacto e limitado.

Teorema 2.1.1. Seja $\left\{T^{n}: n \in \mathbb{N}\right\}$ um semigrupo discreto ponto dissipativo e eventualmente compacto para $n \geqslant n_{1}$. Então $\left\{T^{n}: n \in \mathbb{N}\right\}$ tem um atrator global $\mathcal{A}$.

Demonstração: Segue dos Teoremas 1.2.15 e 1.2.23 que precisamos somente mostrar que as órbitas de conjuntos limitados são eventualmente limitadas; isto é, precisamos mostrar que o semigrupo é eventualmente limitado. Dado um conjunto limitado $B$, segue do fato de que $\left\{T^{n}: n \in \mathbb{N}\right\}$ é eventualmente compacto que existe $n_{B} \in \mathbb{N}$, com $n_{1} \leqslant n_{B}$, tal que $T^{n_{B}}(B)$ é relativamente compacto. Logo, é necessário somente mostrar que a órbita de subconjuntos compactos de $X$ são limitadas, pois $T\left(T^{n_{B}}(B)\right) \subset T\left(\overline{T^{n_{B}}(B)}\right)$.

Seja $K$ um subconjunto compacto de $X$ e $B_{0}$ um subconjunto aberto e limitado de $X$ que absorve pontos. Assim, para cada $x \in K$ existe $n_{x}^{1}>0$ tal que $T^{n_{x}^{1}}(x) \in B_{0}$ e pela continuidade de $T^{n}$ existe uma vizinhança $\mathcal{O}_{x}$ de $x$ tal que $T^{n_{x}^{1}}\left(\mathcal{O}_{x}\right) \subset B_{0}$ e logo, definindo $n_{x}=n_{x}^{1}+n_{B_{0}}$, temos $T^{n_{x}}\left(\mathcal{O}_{x}\right) \subset T^{n_{B_{0}}}\left(B_{0}\right)$. Como $K$ é compacto, existem $\left\{\mathcal{O}_{x_{1}}, \cdots, \mathcal{O}_{x_{p}}\right\}$ que cobrem $K$ e assim $T^{n}(K) \subset T^{n_{B_{0}}}\left(B_{0}\right)$ para $n \geqslant \max \left\{n_{x_{i}}: 1 \leqslant i \leqslant p\right\}$.

Defina $N(K)=\max \left\{n_{x_{i}}: 1 \leqslant i \leqslant p\right\}, K_{0}=\overline{T^{n_{B_{0}}\left(B_{0}\right)}}$ e $\tilde{K}_{0}=\cup_{n=0}^{N\left(K_{0}\right)} T^{n}\left(K_{0}\right)$. Claramente $K_{0}$ e $\tilde{K}_{0}$ são subconjuntos compactos de $X$. Segue que $T^{n}\left(B_{0}\right) \subset \tilde{K}_{0}$ para 
todo $n \geqslant N\left(K_{0}\right)$ e para cada subconjunto compacto $K$ de $X$ temos que $T^{n}(K) \subset \tilde{K}_{0}$ para $n \geqslant N(K)$. Logo

$$
\gamma^{+}(K) \subset\left(\cup_{n=1}^{N(K)} T^{n}(K)\right) \bigcup \tilde{K}_{0},
$$

o que prova que a órbita de um subconjunto compacto de $X$ é limitada e completa a demonstração do nosso resultado.

Teorema 2.1.2. Seja $X$ um espaço de Banach e $\left\{T^{n}: n \in \mathbb{N}\right\}$ um semigrupo discreto em $X$. Assuma que $T^{n}=S_{n}+K_{n}$ com $S_{n}$ e $K_{n}$ satisfazendo

i) Para cada conjunto limitado $B$ em $X$, existe um $n_{B} \in \mathbb{N}$ tal que $K_{n}(B)$ é relativamente compacto para todo $n \geqslant n_{B}$.

ii) Para cada subconjunto limitado $B$ de $X$, existe $n_{B} \in \mathbb{N}$ tal que $\sup _{x \in B}\left\|S_{n}(x)\right\|_{X}:=$ $s_{B}(n)<\infty$ para todo $n \geqslant n_{B}$ e $s_{B}(n) \stackrel{n \rightarrow \infty}{\longrightarrow} 0$.

Então $\left\{T^{n}: n \in \mathbb{N}\right\}$ é assintoticamente compacto. Além disso, se $\left\{T^{n}: n \in \mathbb{N}\right\}$ é ponto dissipativo e eventualmente limitado, então ele possui um atrator global.

Demonstração: Dado um conjunto não-vazio, fechado e limitado $B$ tal que $T(B) \subset B$ e $\epsilon>0$, escolha $n \in \mathbb{N}$ tal que $n \geqslant n_{B}$ e $s_{B}(n)<\epsilon$. Segue que

$$
\omega(B)=\cap_{n=1}^{\infty} \overline{T^{n}(B)} \subset T^{n}(B) \subset S_{n}(B)+K_{n}(B) \subset B_{\epsilon}(0)+K_{n}(B) \subset \mathcal{O}_{\epsilon}\left(K_{n}(B)\right),
$$

e como $K_{n}(B)$ é relativamente compacto, segue do Teorema 1.1 .2 que $\omega(B)$ é relativamente compacto. Podemos ver facilmente que $\omega(B)$ é não-vazio, pois para cada sequência $\left\{x_{j}\right\}$ em $B$ e $n_{j} \in \mathbb{N}$ com $n_{j} \stackrel{j \rightarrow \infty}{\longrightarrow} \infty$ a sequência $\left\{T^{n_{j}}\left(x_{j}\right)\right\}=\left\{K_{n_{j}}\left(x_{j}\right)+S_{n_{j}}\left(x_{j}\right)\right\}$ é totalmente limitada, pois $T^{n_{j}}\left(x_{j}\right) \in T^{n_{j}}(B) \subset T^{n_{k}}(B)$ para todo $j \geqslant k$, possuindo portanto uma subsequência convergente.

Agora, procedendo como na demonstração do Lema 1.2 .9 concluímos que $\omega(B)$ atrai $B$ e isto prova que $\left\{T^{n}: n \in \mathbb{N}\right\}$ é assintoticamente compacto. Em adição, se $\left\{T^{n}: n \in \mathbb{N}\right\}$ é ponto dissipativo e eventualmente limitado, $\left\{T^{n}: n \in \mathbb{N}\right\}$ tem um atrator global.

\subsection{Semicontinuidades superior e inferior de atratores}

Seja $X$ um espaço de Banach, $\Lambda$ um espaço topológico e $\mathcal{A}_{\lambda} \subset X, \lambda \in \Lambda$. Denote por $\operatorname{dist}(\cdot, \cdot): X \times X \rightarrow \mathbb{R}^{+}$a métrica induzida pela norma de $X$. 
Definição 2.2.1. Por semicontinuidade superior e inferior de uma família de conjuntos $\left\{\mathcal{A}_{\lambda}\right\}_{\lambda \in \Lambda}$ em $\lambda=\lambda_{0}$ entenderemos o seguinte

1. Dizemos que $\left\{\mathcal{A}_{\lambda}\right\}$ é semicontínua superiormente em $\lambda_{0}$ se

$$
\sup _{x_{\lambda} \in \mathcal{A}_{\lambda}} \operatorname{dist}\left(x_{\lambda}, \mathcal{A}_{\lambda_{0}}\right) \stackrel{\lambda \rightarrow \lambda_{0}}{\longrightarrow} 0
$$

2. Dizemos que $\left\{\mathcal{A}_{\lambda}\right\}$ é semicontínua inferiormente em $\lambda_{0}$ se

$$
\sup _{x \in \mathcal{A}_{\lambda_{0}}} \operatorname{dist}\left(x, \mathcal{A}_{\lambda}\right) \stackrel{\lambda \rightarrow \lambda_{0}}{\longrightarrow} 0
$$

Para provarmos as semicontinuidades superior e inferior empregaremos o seguinte resultado

Lema 2.2.2. Seja $\left\{\mathcal{A}_{\lambda}\right\}_{\lambda \in \Lambda}$ uma família de conjuntos em um espaço de Banach $X$, onde $\Lambda$ é um espaço topológico.

1. Se qualquer sequência $\left\{x_{\lambda_{n}}\right\}$ com $x_{\lambda_{n}} \in \mathcal{A}_{\lambda_{n}}, \lambda_{n} \stackrel{n \rightarrow \infty}{\longrightarrow} \lambda_{0}$, tem uma subsequência convergente com limite pertencendo a $\mathcal{A}_{\lambda_{0}}$, então $\left\{\mathcal{A}_{\lambda}\right\}_{\lambda \in \Lambda}$ é semicontínua superiormente em $\lambda_{0}$.

2. Se $\mathcal{A}_{\lambda_{0}}$ é compacto e para qualquer $x \in \mathcal{A}_{\lambda_{0}}$ existe uma sequência $\left\{x_{\lambda_{n}}\right\}$ com $x_{\lambda_{n}} \in$ $\mathcal{A}_{\lambda_{n}}, \lambda_{n} \stackrel{n \rightarrow \infty}{\longrightarrow} \lambda_{0}$, que converge para $x$, então $\left\{\mathcal{A}_{\lambda}\right\}_{\lambda \in \Lambda}$ é semicontinua inferiormente em $\lambda_{0}$.

Demonstração: i) Suponha que $\left\{\mathcal{A}_{\lambda}\right\}_{\lambda \in \Lambda}$ não é semicontínua superiormente em $\lambda_{0}$, então existem $\epsilon>0$ e sequência $\left\{\lambda_{n}\right\}$ com $\lambda_{n} \stackrel{n \rightarrow \infty}{\longrightarrow} \lambda_{0}$ tal que $\sup _{x \in \mathcal{A}_{\lambda_{n}}} \operatorname{dist}\left(x, \mathcal{A}_{\lambda_{0}}\right) \geqslant 2 \epsilon, n \in \mathbb{N}$. Logo, para algum $x_{\lambda_{n}} \in \mathcal{A}_{\lambda_{n}}$, temos que $\operatorname{dist}\left(x_{\lambda_{n}}, \mathcal{A}_{\lambda_{0}}\right) \geqslant \epsilon, n \in \mathbb{N}$. Portanto $\left\{x_{\lambda_{n}}\right\}$ não possui nenhuma subsequência que converge para um elemento de $\mathcal{A}_{\lambda_{0}}$, o que contradiz a nossa hipótese.

ii) Se $\mathcal{A}_{\lambda_{0}}$ é compacto e $\left\{\mathcal{A}_{\lambda}\right\}$ não é semicontínua inferiormente em $\lambda_{0}$ então, existem $\epsilon>0$ e sequências $\left\{\lambda_{n}\right\}$ com $\lambda_{n} \stackrel{n \rightarrow \infty}{\longrightarrow} \lambda_{0}$ tais que $\sup _{x \in \mathcal{A}_{\lambda_{0}}} \operatorname{dist}\left(x, \mathcal{A}_{\lambda_{n}}\right) \geqslant 3 \epsilon, n \in \mathbb{N}$. Logo, para algum $x_{\lambda_{n}} \in \mathcal{A}_{\lambda_{0}}$, temos que $\operatorname{dist}\left(x_{\lambda_{n}}, \mathcal{A}_{\lambda_{n}}\right) \geqslant 2 \epsilon, n \in \mathbb{N}$. Como $\mathcal{A}_{\lambda_{0}}$ é compacto podemos assumir que $\left\{x_{\lambda_{n}}\right\}$ converge para algum $x \in \mathcal{A}_{\lambda_{0}}$ e que $\operatorname{dist}\left(x, \mathcal{A}_{\lambda_{n}}\right) \geqslant \epsilon, n \in \mathbb{N}$. 
Agora, se $\left\{y_{\lambda_{n}}\right\}$ é uma sequência qualquer com $y_{\lambda_{n}} \in \mathcal{A}_{\lambda_{n}}$ temos

$$
\epsilon \leqslant \operatorname{dist}\left(x, \mathcal{A}_{\lambda_{n}}\right) \leqslant \operatorname{dist}\left(x, y_{\lambda_{n}}\right)
$$

e assim $\left\{y_{\lambda_{n}}\right\}$ não converge para $x$, o que contraria nossa hipótese.

Teorema 2.2.3 (Semicontinuidade Superior). Seja $[0,1] \ni \eta \mapsto T_{\eta} \in \mathcal{C}(X)$ contínua, uniformemente em subconjuntos compactos de $X$, em $\eta=0$. Se $\left\{T_{\eta}^{n}: n \in \mathbb{N}\right\}$ tem um atrator global $\mathcal{A}_{\eta}$ para cada $\eta \in[0,1]$ e $\overline{\cup_{\eta \in[0,1]} \mathcal{A}_{\eta}}$ é compacto, então a família $\left\{\mathcal{A}_{\eta}: \eta \in\right.$ $[0,1]\}$ é semicontinua superiormente em $\eta=0$.

Demonstração: Considere as subsequências $\eta_{k} \rightarrow 0,\left\{u_{\eta_{k}}\right\}_{k=1}^{\infty}, u_{\eta_{k}} \in \mathcal{A}_{\eta_{k}}$. Como $\overline{\bigcup_{\eta \in[0,1]} \mathcal{A}_{\eta}}$ é compacto em $X$, existe $u_{0} \in X$ tal que $u_{\eta_{k}} \stackrel{k \rightarrow \infty}{\longrightarrow} u_{0}$, a menos de subsequências.

Para concluir a demonstração da semicontinuidade superior falta mostrar que $u_{0} \in \mathcal{A}_{0}$. Para este fim, é suficiente provar que existe uma solução global limitada através de $u_{0}$. Da invariância dos atratores $\mathcal{A}_{\eta_{k}}$, para cada $k \in \mathbb{N}$, existe uma solução global limitada

$$
\begin{aligned}
\psi^{\left(\eta_{k}\right)} & : \mathbb{Z} \\
n & \mapsto \psi_{n}^{\left(\eta_{k}\right)}
\end{aligned}
$$

através de $u_{\eta_{k}}$. Para $n \geqslant 0$, segue da continuidade de $[0,1] \ni \eta \rightarrow T_{\eta} \in \mathcal{C}(X)$ uniformemente em subconjuntos compactos de $X$ que

$$
\psi_{n}^{\left(\eta_{k}\right)}=T_{\eta_{k}}^{n} u_{\eta_{k}} \rightarrow T_{0}^{n} u_{0}
$$

Agora, construímos uma solução para trás através de $u_{0}$ da seguinte maneira. Se $\eta_{k}^{0}:=\eta_{k}, k \in \mathbb{N}$, dado $j \in \mathbb{N}^{*}$ existe uma subsequência $\left\{\eta_{k}^{j}\right\}$ de $\left\{\eta_{k}^{j-1}\right\}$ e $u_{-j}$ tal que $\psi_{-j}^{\left(\eta_{k}^{j}\right)} \stackrel{k \rightarrow \infty}{\longrightarrow} u_{-j}$ (lembremos que $\left\{\psi_{-j}^{\left(\eta_{k}\right)}\right\}_{j \in \mathbb{N}}$ está em $\overline{\bigcup_{\eta \in[0,1]} \mathcal{A}_{\eta}}$ ).

Da convergência de $T_{\eta}$ para $T_{0}$ uniformemente em subconjuntos compactos de $X$ segue que

$$
\psi_{-j}^{\left(\eta_{k}^{j}\right)}=T_{\eta_{k}}^{i} \psi_{-j-i}^{\left(\eta_{k}^{j}\right)} \rightarrow u_{-j}=T_{0}^{i} u_{-j-i}
$$

Definindo

$$
\psi_{j}^{(0)}:= \begin{cases}T_{0}^{j} u_{0}, & \text { se } j \geqslant 0 \\ u_{j}, & \text { se } j<0\end{cases}
$$


temos que $\psi^{(0)}: \mathbb{Z} \rightarrow X$ é uma solução de $\left\{T_{0}^{n}: n \in \mathbb{N}\right\}$ e

$$
\psi^{\left(\eta_{k}^{k}\right)}(j) \stackrel{k \rightarrow \infty}{\longrightarrow} \psi^{(0)}(j), \quad \forall j \in \mathbb{Z}
$$

Como $\psi^{(0)}: \mathbb{Z} \rightarrow X$ é limitada, sua imagem deve estar contida em $\mathcal{A}_{0}$ e em particular $u_{0} \in \mathcal{A}_{0}$. Agora o resultado segue do Lema 2.2.2.

Teorema 2.2.4 (Semicontinuidade Inferior). Seja $\left\{T_{\eta}^{n}: n \in \mathbb{N}\right\}, \eta \in[0,1]$, uma família de semigrupos discretos em um espaço métrico $X$ que satisfaz

a) para cada $\eta \in[0,1],\left\{T_{\eta}^{n}: n \in \mathbb{N}\right\}$ tem um atrator global $\mathcal{A}_{\eta}$;

b) $\left\{T_{\eta}^{n}: n \in \mathbb{N}\right\}$ tem um número finito de soluçôes estacionárias $\mathcal{E}_{\eta}=\left\{y_{1}^{*, \eta}, \cdots, y_{\mathfrak{p}}^{*, \eta}\right\}$, para todo $\eta \in[0,1]$, onde $\mathfrak{p}$ é independente de $\eta$, e $\sup _{1 \leqslant i \leqslant \mathfrak{p}} d\left(y_{i}^{*, \eta}, y_{i}^{*, 0}\right) \stackrel{\eta \rightarrow 0}{\longrightarrow} 0$;

c) existe um $\delta>0$ tal que, se $W_{\mathrm{loc}}^{u}\left(y_{j}^{*, \eta}\right):=W^{u}\left(y_{j}^{*, \eta}\right) \cap B_{\delta}\left(y_{j}^{*, 0}\right)$, então

$$
\left\{W_{\mathrm{loc}}^{u}\left(y_{j}^{*, \eta}\right): \eta \in[0,1]\right\}
$$

é semicontínua inferiormente em $\eta=0$;

d) $d\left(T_{\eta}^{n} u, T_{0}^{n} u\right) \stackrel{\eta \rightarrow 0}{\longrightarrow} 0$ uniformemente para $u$ em subconjuntos compactos de $X$, para $\operatorname{cada} n \in \mathbb{N}$;

e) $\mathcal{A}_{0}=\cup_{j=1}^{\mathfrak{p}} W^{u}\left(y_{j}^{*, 0}\right)$.

Então, $\left\{\mathcal{A}_{\eta}: \eta \in[0,1]\right\}$ é semicontinua inferiormente em $\eta=0$.

Demonstração: Note que primeiramente, de $e), \mathcal{A}_{0}=\cup_{j=1}^{\mathfrak{p}} W^{u}\left(y_{j}^{*, 0}\right)$. Portanto, se $u_{0} \in \mathcal{A}_{0}$, existem um inteiro $\ell \in\{1, \ldots, \mathfrak{p}\}$, uma solução global $\psi_{\ell}^{(0)}: \mathbb{Z} \rightarrow X$ através de $u_{0}$, e um $m \in \mathbb{N}$ tal que $\psi_{\ell}^{(0)}(-m) \in W_{\text {loc }}^{u}\left(y_{\ell}^{*, 0}\right)$. De (c), existe uma solução global $\psi_{\ell}^{(\eta)}: \mathbb{Z} \rightarrow X$ com $\psi_{\ell}^{(\eta)}(-m) \in W_{\text {loc }}^{u}\left(y_{\ell}^{*, \eta}\right)$ tal que $\psi_{\ell}^{(\eta)}(-m) \stackrel{\eta \rightarrow 0}{\longrightarrow} \psi_{\ell}^{(0)}(-m)$. Segue de $\left.a\right)$ e d) que

$$
T_{\eta}^{m} \psi_{\ell}^{(\eta)}(-m) \rightarrow u_{0}
$$

O resultado segue agora do Lema 2.2.2. 


\subsection{Semigrupos discretos gradientes}

Nesta seção vamos considerar os semigrupos discretos gradientes, que são semigrupos aos quais temos associada uma função de Liapunov; isto é, uma função que não cresce ao longo de órbitas. Estes semigrupos discretos gozam da propriedade de que seus atratores podem ser caracterizados como a variedade instável de seu conjunto de equilíbrio. Se em adição, o conjunto de equilíbrio é constituído somente de um número finito de pontos, então o atrator é a união das variedades instáveis de cada um deles.

Lembremos que $x^{*} \in X$ é um ponto de equilíbrio para o semigrupo discreto $\left\{T^{n}\right.$ : $n \in \mathbb{N}\}$ se é um ponto fixo para a aplicação $T$; isto é, $T x^{*}=x^{*}$. Denotaremos por $\mathcal{E}$ o conjunto dos pontos de equilíbrio para o semigrupo discreto $\left\{T^{n}: n \in \mathbb{N}\right\}$.

Definição 2.3.1. Um semigrupo discreto $\left\{T^{n}: n \in \mathbb{N}\right\}$ é dito um semigrupo gradiente se tem uma função de Liapunov ; isto é, se existe uma função contínua $V: X \rightarrow \mathbb{R}$ com as seguintes propriedades:

(i) $\mathbb{N} \ni n \mapsto V\left(T^{n} x\right)$ é não-crescente para cada $x \in X$;

(ii) Se $x$ é tal que $V(T x)=V(x)$, então $x \in \mathcal{E}$.

Para semigrupos discretos gradientes temos o seguinte resultado de caracterização:

Lema 2.3.2. Se $\left\{T^{n}: n \in \mathbb{N}\right\}$ é um semigrupo discreto gradiente, então $\omega(x)$ é um subconjunto de $\mathcal{E}$ para cada $x \in X$. Se existe uma solução para trás $\phi: \mathbb{Z}^{-} \rightarrow X$ através de $x$ então $\alpha_{\phi}(x)$ é um subconjunto de $\mathcal{E}$. Se além disso $\left\{T^{n}: n \in \mathbb{N}\right\}$ possui um atrator global $\mathcal{A}$ e todas as soluções estacionárias são isoladas, existe somente um número finito delas e para cada $x \in X, \omega(x)$ é um conjunto unitário. Se $x \in \mathcal{A}$ e $\phi: \mathbb{Z} \rightarrow X$ é uma solução global através de $x, \alpha_{\phi}(x)$ é um conjunto unitário.

Demonstração: Se $\omega(x)=\emptyset$, o resultado é trivial. Se $\omega(x) \neq \emptyset$ e $y \in \omega(x)$ temos que $V\left(T^{n}(x)\right) \stackrel{n \rightarrow \infty}{\longrightarrow} c$ para algum $c \in \mathbb{R}$, pois é uma sequência não-crescente com uma subsequência convergente, além disso $V(y)=c$. Como $T(\omega(x)) \subset \omega(x)$ temos que cada ponto $y \in \omega(x)$ é tal que $V(T(y))=V(y)=c$ e da propriedade (ii) na Definição 2.3.1 temos que $y \in \mathcal{E}$.

Suponha que exista uma solução para trás $\phi: \mathbb{Z}^{-} \rightarrow X$ através de $x$. Se $\alpha_{\phi}(x)=\emptyset_{\mathrm{o}}$ resultado é trivial. Se $y \in \alpha_{\phi}(x)$, existe um $c \in \mathbb{R}$ tal que $V(\phi(-n)) \stackrel{n \rightarrow \infty}{\longrightarrow} c$, pois é uma 
sequência não-decrescente com uma subsequência convergente. Como $T\left(\alpha_{\phi}(x)\right) \subset \alpha_{\phi}(x)$ temos que para cada $y \in \alpha_{\phi}(x), V(T(y))=V(y)=c$ e $y \in \mathcal{E}$.

Agora assumamos que $\left\{T^{n}: n \in \mathbb{N}\right\}$ tem um atrator global $\mathcal{A}$. Como $\mathcal{A}$ é compacto e $\mathcal{E} \subset \mathcal{A}$, segue que se todos os pontos de $\mathcal{E}$ são isolados, então $\mathcal{E}$ é finito.

Falta ainda mostrar que se o conjunto das soluções estacionárias é finito então $\omega(x)$ e $\alpha_{\phi}(x)$ são conjuntos unitários. Se assumirmos que $\omega(x)=\left\{y_{1}^{*}, \cdots, y_{\ell}^{*}\right\} \subset \mathcal{E}$ com $\ell \geqslant 2$, então existe uma cobertura disjunta $\left\{\mathbb{N}_{i}: 1 \leqslant i \leqslant \ell\right\}$ de $\mathbb{N}$ com a propriedade de que cada $\mathbb{N}_{i}$ é infinito e $\lim _{\substack{n \in N_{i} \\ n \rightarrow \infty}} T^{n}(x)=y_{i}^{*}, 1 \leqslant i \leqslant \ell$. Escolha uma sequência $\left\{k_{n}: n \in \mathbb{N}\right\}$ tal que $k_{2 n-1} \in \mathbb{N}_{1}$ e $k_{2 n}=k_{2 n-1}+1 \notin \mathbb{N}_{1}$. Então, a menos de subsequênciais, $y_{1}^{*}=T\left(y_{1}^{*}\right)=$ $\lim _{k \rightarrow \infty} T^{k_{2 n}}(x)=y_{j}^{*}$, para algum $2 \leqslant j \leqslant \ell$, o que é uma contradição. Isto mostra que se o conjunto das soluções estacionárias for finito, então $\omega(x)$ é um conjunto unitário. A prova de que $\alpha_{\phi}(x)$ é também um conjunto unitário é inteiramente análoga a de $\omega(x)$, e assim concluímos a demonstração.

Teorema 2.3.3. Assuma que $\left\{T^{n}: n \in \mathbb{N}\right\}$ é um semigrupo discreto gradiente que é eventualmente limitado, assintoticamente compacto e tem um conjunto de equilíbrio $\mathcal{E}$ limitado. Então $\left\{T^{n}: n \in \mathbb{N}\right\}$ tem um atrator global $\mathcal{A}=W^{u}(\mathcal{E})$, onde

$W^{u}(\mathcal{E}):=\{y \in X:$ existe uma solução para trás

$$
\left.\phi(\cdot, y): \mathbb{Z}^{-} \rightarrow X \text { através de y tal que } \phi(n, y) \stackrel{n \rightarrow-\infty}{\longrightarrow} \mathcal{E}\right\}
$$

é chamado de conjunto instável de $\mathcal{E}$. Se $\mathcal{E}=\left\{e_{1}^{*}, \cdots, e_{n}^{*}\right\}$ é finito então $\mathcal{A}=\cup_{i=1}^{n} W^{u}\left(e_{i}^{*}\right)$ onde

$$
\begin{aligned}
& W^{u}\left(e_{i}^{*}\right):=\{y \in X \text { : existe uma solução para trás } \\
& \left.\qquad \phi(\cdot, y): \mathbb{Z}^{-} \rightarrow X \text { através de } y \text { tal que } \phi(n, y) \stackrel{n \rightarrow-\infty}{\longrightarrow} e_{i}^{*}\right\}
\end{aligned}
$$

é o conjunto instável de $e_{i}^{*}$. Finalmente, se existe um conjunto conexo e limitado $B$ que contém $\mathcal{A}$, então $\mathcal{A}$ é conexo.

Demonstração: Como $\left\{T^{n}: n \in \mathbb{N}\right\}$ é eventualmente limitado e assintoticamente compacto temos do Lema 1.2 .13 que, para cada $x \in X, \omega(x)$ é não-vazio, compacto, invariante e atrai $x$. Do fato de que $\left\{T^{n}: n \in \mathbb{N}\right\}$ é gradiente temos que $\omega(x) \subset \mathcal{E}$ e como $\mathcal{E}$ é limitado temos que $\left\{T^{n}: n \in \mathbb{N}\right\}$ é ponto dissipativo. Logo, do Teorema 1.2.23, segue que $\left\{T^{n}: n \in \mathbb{N}\right\}$ tem um atrator global. 
Se $x \in \mathcal{A}$, existe uma solução global $\phi: \mathbb{Z} \rightarrow X$ através de $x$. Como $\phi(\mathbb{Z}) \subset \mathcal{A}$ é relativamente compacto, $\alpha_{\phi}(x) \neq \emptyset$. Do Lema 2.3.2, $\alpha_{\phi}(x) \subset \mathcal{E}$. Isto mostra que $\mathcal{A} \subset W^{u}(\mathcal{E})$. Se $x \in W^{u}(\mathcal{E})$, existe uma solução global $\phi: \mathbb{Z} \rightarrow X$ através de $x$ e $\phi(n) \stackrel{n \rightarrow \pm \infty}{\longrightarrow} \mathcal{E} \subset \mathcal{A}$. Do fato de que $\phi(\mathbb{Z})$ é invariante concluímos que $\phi(\mathbb{Z}) \subset \mathcal{A}$ e consequentemente $x \in \mathcal{A}$. Isto mostra que $\mathcal{A} \supset W^{u}(\mathcal{E})$ e completa a prova de que $\mathcal{A}=W^{u}(\mathcal{E})$.

Se $\mathcal{E}=\left\{e_{1}^{*}, \cdots e_{n}^{*}\right\}$, a igualdade $\mathcal{A}=\cup_{i=1}^{n} W^{u}\left(e_{i}^{*}\right)$ segue imediatamente do Lema 2.3 .2 e se $\mathcal{A}$ está contido em um subconjunto conexo e limitado de $X$ o Lema 1.2 .7 implica que $\mathcal{A}$ é conexo.

Lema 2.3.4. Assuma que $\left\{T^{n}: n \in \mathbb{N}\right\}$ é um semigrupo discreto gradiente que tem um atrator global $\mathcal{A}$ e tal que $T$ tem um número finito de pontos fixos $\mathcal{E}=\left\{y_{i}^{*}: 1 \leqslant i \leqslant n\right\}$. Seja $V: X \rightarrow \mathbb{R}$ a função de Liapunov associada à $\left\{T^{n}: n \in \mathbb{N}\right\}$ e $V(\mathcal{E})=\left\{\mathfrak{n}_{1}, \cdots, \mathfrak{n}_{p}\right\}$ com $\mathfrak{n}_{i}<\mathfrak{n}_{i+1}, 1 \leqslant i \leqslant p-1$.

Se $1 \leqslant j \leqslant p-1$ e $\mathfrak{n}_{j} \leqslant r<\mathfrak{n}_{j+1}$, então $X_{r}=\{z \in X: V(z) \leqslant r\}$ é positivamente invariante sobre $\left\{T^{n}: n \in \mathbb{N}\right\}$ e $\left\{T_{r}^{n}: n \in \mathbb{N}\right\}$, a restrição de $\left\{T^{n}: n \in \mathbb{N}\right\}$ a $X_{r}$, tem atrator global $\mathcal{A}^{(j)}$ dado por

$$
\mathcal{A}^{(j)}=\cup\left\{W^{\mathrm{u}}\left(y_{\ell}^{*}\right): V\left(y_{\ell}^{*}\right) \leqslant \mathfrak{n}_{j}\right\}
$$

Em particular, $V(z) \leqslant \mathfrak{n}_{j}$ para $z \in \mathcal{A}^{(j)}, \mathfrak{n}_{1}=\min \{V(x): x \in X\}$ e $\mathcal{A}^{(1)}=\left\{x^{*} \in \mathcal{E}:\right.$ $\left.V\left(x^{*}\right)=\mathfrak{n}_{1}\right\}$ consiste de todos os pontos de equilíbrio assintoticamente estáveis; isto é para cada $x^{*} \in \mathcal{A}^{(1)}$ existe uma vizinhança $\mathcal{O}_{x^{*}}$ de $x^{*}$ tal que $T^{n} x \rightarrow x^{*}$ para cada $x \in \mathcal{O}_{x^{*}}$.

Demonstração: É claro da definição da função de Liapunov que $X_{r}$ é positivamente invariante sobre $\left\{T^{n}: n \in \mathbb{N}\right\}$. Para provar a existência de um atrator para $\left\{T_{r}^{n}: n \in \mathbb{N}\right\}$ notemos que as propriedades requeridas para obtermos um atrator global são herdadas de $\left\{T^{n}: n \in \mathbb{N}\right\}$; a saber, órbitas de subconjuntos limitados de $X_{r}$ são limitadas, $\left\{T_{r}^{n}: n \in\right.$ $\mathbb{N}\}$ é ponto dissipativo e $\left\{T_{r}^{n}: n \in \mathbb{N}\right\}$ é assintoticamente compacto. Logo, $\left\{T_{r}^{n}: n \in \mathbb{N}\right\}$ tem um atrator global $\mathcal{A}^{(j)}$. A restrição $V_{r}$ de $V$ à $X_{r}$ é uma função de Liapunov para $\left\{T_{r}^{n}: n \in \mathbb{N}\right\}$ e a caracterização de $\mathcal{A}^{(j)}$ segue.

Agora provemos a última afirmação. Seja $\delta_{0}=\frac{1}{2} \min \left\{d\left(x^{*}, y^{*}\right), x^{*}, y^{*} \in \mathcal{A}^{(1)}, x^{*} \neq\right.$ $\left.y^{*}\right\}$. Se existem um $\delta_{0}>\delta>0$ e sequências $\left\{x_{k}: k \in \mathbb{N}\right\}$ em $X$ e $\left\{n_{k}: k \in \mathbb{N}\right\}$ em $\mathbb{N}$ tais que $x_{k} \stackrel{k \rightarrow \infty}{\longrightarrow} x^{*}$ e $d\left(T^{n_{k}} x_{k}, \mathcal{A}^{(1)}\right) \geqslant \delta$ concluímos que $\left\{T^{n_{k}} x_{k}: k \in \mathbb{N}\right\}$ tem uma 
subsequência convergente.

De fato, se $\left\{n_{k}: k \in \mathbb{N}\right\}$ é limitado, segue da continuidade de $T$ e de suas iteradas que $\left\{T^{n_{k}} x_{k}: k \in \mathbb{N}\right\}$ tem uma subsequência convergente. Agora, se $\left\{n_{k}: k \in \mathbb{N}\right\}$ não é limitada, como $\left\{x_{k}: k \in \mathbb{N}\right\}$ é limitada, existe $n \in \mathbb{N}$ tal que $\gamma^{+}\left(\left\{x_{k}: k \in \mathbb{N}\right\}\right)$ é limitada. Da compacidade assintótica do semigrupo, existe um compacto $K \subset \overline{\gamma^{+}\left(\left\{x_{k}: k \in \mathbb{N}\right\}\right)}$ que atrai $\overline{\gamma^{+}\left(\left\{x_{k}: k \in \mathbb{N}\right\}\right)}$, e consequentemente atrai $\left\{T^{n_{k}} x_{k}: k \in \mathbb{N}\right\}$, portanto $\left\{T^{n_{k}} x_{k}\right.$ : $k \in \mathbb{N}\}$ tem uma subsequência convergente. Denote esta subsequência convergente por $\left\{T^{n_{k}} x_{k}: k \in \mathbb{N}\right\}$ e seja $y$ seu limite.

É imediato do fato de que $V\left(x_{k}\right) \rightarrow \mathfrak{n}_{1}$ que $V(y)=V(T y)=\mathfrak{n}_{1}$, pois $\mathfrak{n}_{1} \leqslant V(T y) \leqslant$ $V(y) \leqslant \mathfrak{n}_{1}$. Portanto $y \in \mathcal{A}^{(1)}$ e $\operatorname{dist}\left(y, \mathcal{A}_{1}\right) \geqslant \delta$, o que é um absurdo. Isto prova que, para cada $x^{*} \in \mathcal{A}^{(1)}$ e $0<\delta<\delta_{0}$ existe um $\delta>\delta^{\prime}>0$ tal que, para todo $x \in B_{\delta^{\prime}}\left(x^{*}\right)$, $\gamma^{+}(x) \subset B_{\delta}\left(x^{*}\right)$ e prova que $\mathcal{A}^{(1)}$ consiste somente dos equilíbrios estáveis. Para concluir precisamos somente notar que, para cada $x \in X, T^{n}(x) \stackrel{n \rightarrow \infty}{\longrightarrow} x^{*}$ para algum $x^{*} \in \mathcal{E}$.

\subsection{Semigrupos discretos gradient-like}

Em geral não esperamos que uma perturbação de um semigrupo discreto gradiente nos dê um novo semigrupo gradiente, e a maior dificuldade é provar que o problema perturbado tem uma função de Liapunov. Assim, definimos o conceito de semigrupo gradient-like, onde nos preocupamos somente com as propriedades dinâmicas que a função de Liapunov introduz num semigrupo discreto gradiente. Com esta nova definição mostramos que nesta nova classe os atratores podem ser caracterizados como nos semigrupos gradientes e também mostramos que este conceito é estável sob pequenas perturbações, logo conseguimos mostrar a continuidade da estrutura dos atratores para este tipo de semigrupos com relação à pequenas perturbações.

Antes de continuarmos, vamos enfatizar a distinção entre semigrupos discretos gradientes e semigrupos que têm atratores do tipo gradiente. Como na Definição 2.3.1, um semigrupo discreto gradiente é um semigrupo que possui uma função de Liapunov.

Definição 2.4.1. Seja $\left\{T^{n}: n \in \mathbb{N}\right\}$ um semigrupo discreto com um atrator global $\mathcal{A}$. Assuma que o conjunto das soluções estacionárias $\mathcal{E}$ de $\left\{T^{n}: n \in \mathbb{N}\right\}$ é finito; isto é, para algum $\mathfrak{p} \in \mathbb{N}, \mathcal{E}=\left\{y_{1}^{*}, \cdots, y_{\mathfrak{p}}^{*}\right\}$. Se $\mathcal{A}=\cup_{i=1}^{\mathfrak{p}} W^{u}\left(y_{i}^{*}\right)$, dizemos que $\mathcal{A}$ é um atrator do tipo gradiente e que $\left\{T^{n}: n \in \mathbb{N}\right\}$ é um semigrupo discreto com atrator do tipo gradiente. 
Como provado no Teorema 2.3.3, um semigrupo discreto gradiente com um atrator global e um número finito de equilíbrios é um semigrupo discreto com um atrator do tipo gradiente.

Não é difícil ver (veja exemplo em Hale [12 páginas 2 e 3) que um atrator do tipo gradiente pode vir de um semigrupo discreto que não é gradiente e também que uma perturbação de um semigrupo discreto com um atrator do tipo gradiente pode não ter atrator do tipo gradiente.

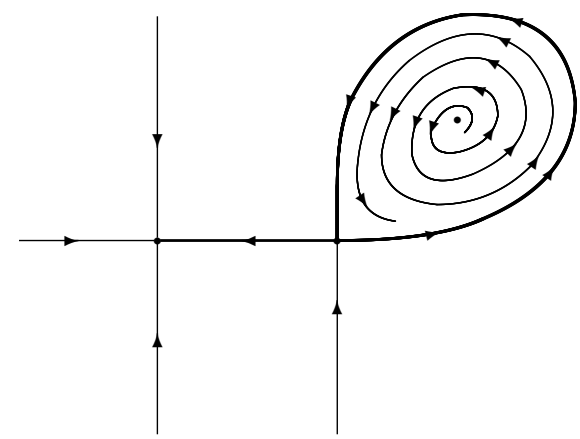

Figura 01

A Figura 1 acima apresenta um atrator do tipo gradiente que não é proveniente de um semigrupo gradient-like. Mas uma pequena perturbação deste semigrupo nos dá

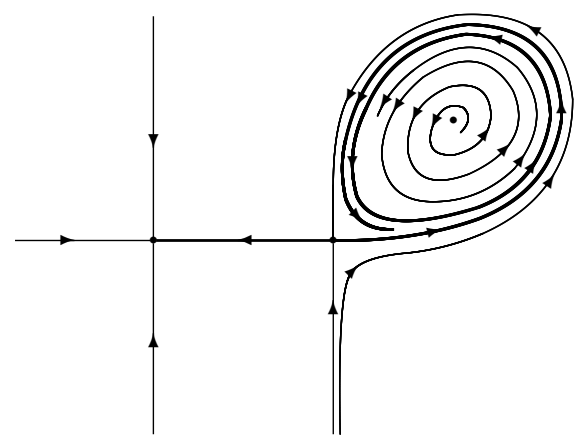

Figura 02

e temos assim uma órbita periódica, o que mostra que o atrator deste semigrupo não é a união dos conjuntos instáveis de seus pontos de equilíbrio. Notemos também que a perturbação não alterou o comportamento dos atratores do ponto de vista de semicontinuidade superior e inferior, mas alterou sua estrutura.

Em Carvalho-Langa-Robinson-Suárez [5] os autores provam que uma perturbação de um semigrupo gradiente tem atrator do tipo gradiente. As provas são imediatamente adaptadas para semigrupos discretos. Infelizmente, semigrupos que têm atratores do tipo gradiente não precisam ser gradientes. 
Isto nos leva à questão de quais propriedades dinâmicas dos semigrupos discretos garantem que eles tenham atratores do tipo gradiente e que são estáveis sobre perturbações. Logicamente, tais propriedades devem ser satisfeitas por semigrupos discretos gradientes e sua perturbações.

Com isto em mente (veja Carvalho-Langa [4]) o seguinte conceito de semigrupos discretos gradient-like generaliza o conceito de semigrupos discretos gradientes enquanto mantém suas propriedades dinâmicas essenciais. Provaremos agora, adaptando os resultados de Carvalho-Langa [4] para o caso discreto, que as propriedades que definem os semigrupos discretos gradient-like são estáveis por perturbações.

Definição 2.4.2. Considere um semigrupo discreto $\left\{T^{n}: n \in \mathbb{N}\right\}$ com um número finito de soluções estacionárias $\mathcal{E}=\left\{y_{1}^{*}, \cdots y_{\mathfrak{p}}^{*}\right\}$. Seja

$$
2 \delta_{0}=\min _{\substack{1 \leqslant i, j \leqslant \mathfrak{p} \\ i \neq j}} d\left(y_{i}^{*}, y_{j}^{*}\right)>0
$$

Seja $\epsilon_{0}<\delta_{0}, y^{*} \in \mathcal{E}$ e $\epsilon \in\left(0, \epsilon_{0}\right)$. Uma $\epsilon$-cadeia de $y^{*}$ a $y^{*}$ é uma sequência $\left\{y_{\ell_{1}}^{*}, \ldots, y_{\ell_{k}}^{*}\right\}$ em $\mathcal{E}$, juntamente com um conjunto $\left\{y_{1}, \cdots, y_{k}\right\}$ de pontos de $X$ e inteiros $\left\{n_{1}, \kappa_{1}, \ldots, n_{k}, \kappa_{k}\right\}$, $0<\kappa_{i}<n_{i}, 1 \leqslant i \leqslant k, k \leq \mathfrak{p}$, tais que $d\left(y_{i}, y_{\ell_{i}}^{*}\right)<\epsilon, 1 \leqslant i \leqslant k+1, y^{*}=y_{\ell_{1}}^{*}=y_{\ell_{k+1}}^{*}$, $\operatorname{dist}\left(T^{\kappa_{i}} y_{i}, \mathcal{E}\right)>\epsilon_{0}$ e d $\left(T^{n_{i}} y_{i}, y_{\ell_{i+1}}^{*}\right)<\epsilon, 1 \leqslant i \leqslant k$. Dizemos que $y^{*} \in \mathcal{E}$ é recorrente por cadeias se existe um $\epsilon_{0}>0$ fixo e uma $\epsilon$-cadeia de $y^{*}$ a $y^{*}$, para cada $\epsilon \in\left(0, \epsilon_{0}\right)$.
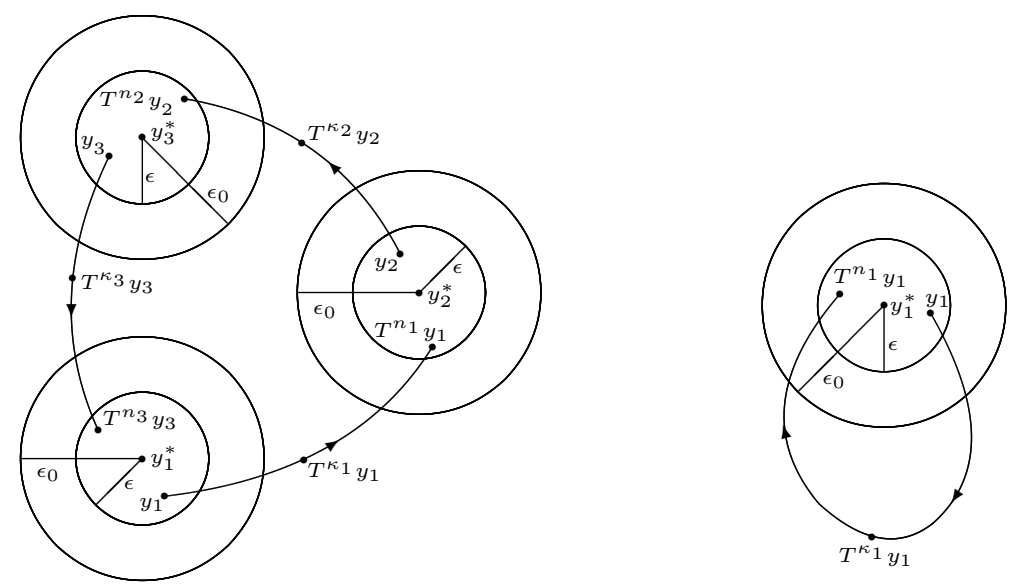

Exemplos de $\epsilon$-cadeias 
Definição 2.4.3. Seja $\left\{T^{n}: n \in \mathbb{N}\right\}$ um semigrupo discreto com um número finito de soluções estacionárias $\mathcal{E}=\left\{y_{1}^{*}, \cdots y_{\mathfrak{p}}^{*}\right\}$ e assuma que ele tem um atrator global $\mathcal{A}$. Dizemos que $\left\{T^{n}: n \in \mathbb{N}\right\}$ é um semigrupo discreto gradient-like se as seguintes condições são satisfeitas:

(G1) Dada uma solução global $\xi: \mathbb{Z} \rightarrow X$ em $\mathcal{A}$, existem $i, j \in\{1, \cdots, \mathfrak{p}\}$ tais que

$$
\lim _{n \rightarrow-\infty} d\left(\xi_{n}, y_{i}^{*}\right)=0 e \lim _{n \rightarrow \infty} d\left(\xi_{n}, y_{j}^{*}\right)=0 .
$$

$(G 2) \mathcal{E}=\left\{y_{1}^{*}, \cdots, y_{\mathfrak{p}}^{*}\right\}$ não contém nenhum ponto recorrente por cadeia.

As hipóteses $(G 1)$ e $(G 2)$ carregam importantes propriedades dinâmicas de um semigrupo discreto com uma função de Liapunov (veja também os Lemas 2.4.5 e 2.4.6 abaixo). De $(G 1)$, temos que $\mathcal{A}=\cup_{i=1}^{\mathfrak{p}} W^{u}\left(y_{i}^{*}\right)$, pois se $x \in \mathcal{A}$ então existe uma solução global $\xi: \mathbb{Z} \rightarrow X$ em $\mathcal{A}$ e assim $x \in W^{u}\left(y_{i}^{*}\right)$, para algum $1 \leqslant i \leqslant \mathfrak{p}$ e reciprocamente, se $x \in W^{u}\left(y_{i}^{*}\right)$ para algum $1 \leqslant i \leqslant \mathfrak{p}$ então, como $\mathcal{A}$ atrai pontos, existe uma órbita global limitada através de $x$, assim $x \in \mathcal{A}$. Também, a hipótese $(G 2)$ diz que nenhum número finito de órbitas pode produzir um contorno fechado.

Os seguintes lemas são de fundamental importância para o futuro desenvolvimento da teoria de atratores para semigrupos discretos gradient-like.

O seguinte resultado segue imediatamente da continuidade das iteradas de uma aplicação $T \in \mathcal{C}(X)$. Ele garante que, dado um ponto fixo $x^{*}$ de $T$ e $y$ perto de $x^{*}$, a órbita finita $\gamma_{[0, n]}(y)=\left\{y, T y, \cdots, T^{n} y\right\}$ permanece perto de $x^{*}$ para grandes valores de $n$.

Lema 2.4.4. Seja $T \in \mathcal{C}(X)$ e $x^{*}$ um ponto fixo de $T$. Dado $n \in \mathbb{N}$ e $\epsilon>0$, existe $\delta>0$ tal que $\left\{T^{j} y: 0 \leqslant j \leqslant n, y \in B_{\delta}\left(x^{*}\right)\right\} \subset B_{\epsilon}\left(x^{*}\right)$.

Demonstração: Basta notar que a família de aplicações $\left\{I, T, T^{2}, \cdots, T^{n}\right\}$ é equicontínua em $x^{*}$.

Nosso próximo resultado garante que, para um semigrupo discreto gradient-like $\left\{T^{n}\right.$ : $n \in \mathbb{N}\}$, dado um subconjunto limitado $B$ de $X$ e uma vizinhança $\mathcal{N}(\mathcal{E})$ do conjunto de equilíbrio $\mathcal{E}$, existe um inteiro positivo $n_{0}=n_{0}(B, \mathcal{N}(\mathcal{E}))$ tal que todas as soluções começando em pontos de $B$, visitam $\mathcal{N}(\mathcal{E})$ antes do instante $n_{0}$. 
Lema 2.4.5. Seja $\left\{T^{n}: n \in \mathbb{N}\right\}$ um semigrupo discreto com um número finito de soluções estacionárias $\mathcal{E}=\left\{y_{1}^{*}, \cdots y_{\mathfrak{p}}^{*}\right\}$ e assuma que ele tem um atrator global $\mathcal{A}$. Se $\left\{T^{n}: n \in \mathbb{N}\right\}$ satisfaz (G1), dado $\delta<\delta_{0}$ e $B \subset X$ limitado, existe um $n_{0}=n_{0}(\delta, B)>0$ tal que $\left\{T^{n} u_{0}: 0 \leqslant n \leqslant n_{0}\right\} \cap \cup_{i=1}^{\mathfrak{p}} B_{\delta}\left(y_{i}^{*}\right) \neq \emptyset$ para todo $u_{0} \in B$.

Demonstração: Provaremos o resultado por contradição. Assuma que existe uma sequência $\left\{u_{k}\right\}_{k \in \mathbb{N}}$ em $B$ e uma sequência de números naturais $\left\{n_{k}\right\}$ (com $n_{k} \stackrel{k \rightarrow \infty}{\longrightarrow} \infty$ ) tais que $\left\{T^{j} u_{k}: 0 \leqslant j \leqslant 2 n_{k}\right\} \cap \cup_{i=1}^{\mathfrak{p}} B_{\delta}\left(y_{i}^{*}\right)=\emptyset$. Extraindo subsequências, temos que existe uma solução global $\xi: \mathbb{Z} \rightarrow X$ tal que $T^{j+n_{k}} u_{k} \stackrel{k \rightarrow \infty}{\longrightarrow} \xi_{j}$ para $j \in \mathbb{Z}$. Claramente $\xi_{j} \in \mathcal{A}$, para todo $j \in \mathbb{Z}$ e como para $-n_{k} \leqslant j \leqslant n_{k}, T^{j+n_{k}} u_{k} \notin \cup_{i=1}^{\mathfrak{p}} B_{\delta}\left(y_{i}^{*}\right), \xi_{j} \notin \cup_{i=1}^{\mathfrak{p}} B_{\delta}\left(y_{i}^{*}\right)$ para todo $j \in \mathbb{Z}$, o que contradiz $(G 1)$.

A seguir provaremos uma resultado afirmando que, para um semigrupo discreto gradiente-like $\left\{T^{n}: n \in \mathbb{N}\right\}$, dada uma vizinhaça $\mathcal{N}_{2}\left(x^{*}\right)$ de um ponto de equilíbrio $x^{*}$, existe outra vizinhança $\mathcal{N}_{1}\left(x^{*}\right)$ de $x^{*}$ tal que se uma solução começa em $\mathcal{N}_{1}\left(x^{*}\right)$ e deixa $\mathcal{N}_{2}\left(x^{*}\right)$, então ela nunca volta para $\mathcal{N}_{1}\left(x^{*}\right)$.

Lema 2.4.6. Seja $\left\{T^{n}: n \in \mathbb{N}\right\}$ um semigrupo discreto gradient-like. Denote por $\mathcal{E}=$ $\left\{y_{1}^{*}, \cdots y_{\mathfrak{p}}^{*}\right\}$ suas soluções estacionárias e por $\mathcal{A}$ seu atrator global. Então, dado $0<\delta<\delta_{0}$, existe um $\delta^{\prime}>0$ tal que, se para algum $1 \leqslant i \leqslant \mathfrak{p}$, $d\left(u_{0}, y_{i}^{*}\right)<\delta^{\prime}$ e, para algum $n_{1}>0$, $d\left(T^{n_{1}} u_{0}, y_{i}^{*}\right) \geqslant \delta$, então $d\left(T^{n} u_{0}, y_{i}^{*}\right)>\delta^{\prime}$ para todo $n \geqslant n_{1}$.

Demonstração: Assuma que, para algum $1 \leqslant i \leqslant \mathfrak{p}$, existe uma sequência $\left\{u_{k}\right\}_{k \in \mathbb{N}}$ em $X \operatorname{com} d\left(u_{k}, z_{i}^{*}\right)<\frac{1}{k}$ e sequências $n_{k}<m_{k}$ de números positivos tais que $d\left(T^{n_{k}} u_{k}, z_{i}^{*}\right) \geqslant \delta$ e $d\left(T^{m_{k}} u_{k}, z_{i}^{*}\right)<\frac{1}{k}$. Isto contradiz $(G 2)$.

Agora provaremos que, para um semigrupo discreto gradient-like, o $\omega$-limite de um ponto consiste exatamente dos pontos de equilíbrio. Notemos que a condição $\left(G_{1}\right)$ é imposta apenas para soluções no atrator.

Lema 2.4.7. Assuma que $\left\{T^{n}: n \geqslant 0\right\}$ é um semigrupo discreto gradient-like com um conjunto de equilíbrio $\mathcal{E}=\left\{y_{1}^{*}, \cdots, y_{\mathfrak{p}}^{*}\right\}$ e um atrator global $\mathcal{A}$. Dado $u \in X$ existe um $y_{j}^{*} \in \mathcal{E}$ tal que

$$
T^{n} u \stackrel{n \rightarrow \infty}{\longrightarrow} y_{j}^{*}
$$

Demonstração: Segue do Lema 2.4.6 que, dado $r \in \mathbb{N}^{*}$ existe $r^{\prime} \in \mathbb{N}^{*}$ tal que $d\left(v, y_{i}^{*}\right)<\frac{1}{r^{\prime}}$ e se para algum $n_{r}>0, d\left(T^{n_{r}} v, y_{i}^{*}\right) \geqslant \frac{1}{r}$, então $d\left(T^{n} v, y_{i}^{*}\right)>\frac{1}{r^{\prime}}$ para todo $n \geqslant n_{r}$. Por 
outro lado, segue do Lema 2.4.5 que, dado $r^{\prime} \in \mathbb{N}^{*}$ existe um $k_{r^{\prime}}=k_{r^{\prime}}\left(\gamma^{+}(u)\right) \in \mathbb{N}$ tal que, para cada $v \in \gamma^{+}(u)$,

$$
\left\{v, T v, \cdots, T^{k_{r^{\prime}}} v\right\} \cap \cup_{i=1}^{\mathfrak{p}} B_{\frac{1}{r^{\prime}}}\left(y_{i}^{*}\right) \neq \emptyset
$$

pois $\gamma^{+}(u)$ é limitado. Segue do fato de que $\mathcal{E}$ é finito que existe um $y_{j}^{*} \in \mathcal{E}$ e, para cada $r \in \mathbb{N}^{*}$, um $\ell_{r} \in \mathbb{N}$ tal que $T^{\ell} u \in B_{\frac{1}{r}}\left(y_{j}^{*}\right)$ para todo $\ell \geqslant \ell_{r}$. Isto completa a prova do resultado.

Observação 2.4.8. Claramente, não é necessário considerar a solução para trás análoga à do lema acima pois neste caso teríamos uma solução global limitada, consequentemente uma solução global no atrator, e o resultado seguiria diretamente da propriedade (G1).

Antes de prosseguirmos com a análise dos atratores dos semigrupos discretos gradientlike sobre perturbações vamos estabelecer a equivalência entre a condição $(G 2)$ e a ausência de estruturas homoclínicas. Primeiro vamos mostrar o seguinte importante resultado

Proposição 2.4.9. Assuma que $\left\{T^{n}: n \in \mathbb{N}\right\}$ é um semigrupo discreto assintoticamente compacto. Sejam $\left\{s_{k}\right\}_{k \in \mathbb{N}},\left\{n_{k}\right\}_{k \in \mathbb{N}}$ sequências em $\mathbb{N}$ com $s_{k}, n_{k} \stackrel{k \rightarrow \infty}{\longrightarrow} \infty$ e seja $\left\{u_{k}\right\}_{k \in \mathbb{N}}$ uma sequência limitada em $X$. Para $\mathbb{J}_{k}=\left\{j \in \mathbb{Z}:-s_{k} \leqslant j \leqslant n_{k}\right\}$, defina $\xi^{k}: \mathbb{J}_{k} \rightarrow X$ por $\xi^{k}(j)=T^{j+s_{k}} u_{k}, j \in \mathbb{J}_{k} . S e\left\{T^{j} u_{k}: k \in \mathbb{N}, 0 \leqslant j \leqslant s_{k}+n_{k}\right\}$ é limitada, existe uma solução global $y: \mathbb{Z} \rightarrow X$ de $\left\{T^{n}: n \in \mathbb{N}\right\}$ e uma subsequência de $\left\{\xi^{k}\right\}_{k \in \mathbb{N}}$ (que novamente denotamos por $\left.\left\{\xi^{k}\right\}_{k \in \mathbb{N}}\right)$ tal que

$$
\lim _{k \rightarrow \infty} \xi^{k}(j) \rightarrow y(j), \quad \forall j \in \mathbb{Z}
$$

A demonstração da Proposição 2.4 .9 é imediata do Lema 2.4.15 (que será demonstrado ainda nesta seção) no caso particular quando a família de semigrupos discretos é independente do parâmetro.

Definição 2.4.10. Seja $\left\{T^{n}: n \in \mathbb{N}\right\}$ um semigrupo discreto com um número finito de soluções estacionárias $\mathcal{E}=\left\{y_{1}^{*}, \cdots y_{\mathfrak{p}}^{*}\right\}$ e assuma que ele tenha um atrator global $\mathcal{A}$. Uma estrutura homoclínica em $\mathcal{A}$ é um conjunto $\left\{y_{\ell_{1}}^{*}, \cdots, y_{\ell_{k}}^{*}\right\} \subset \mathcal{E}$ e um conjunto de soluções 
globais $\left\{\xi^{(i)}: \mathbb{Z} \rightarrow X, 1 \leqslant i \leqslant k\right\}$ em $\mathcal{A}$ tal que, fazendo $y_{\ell_{k+1}}^{*}:=y_{\ell_{1}}^{*}$,

$$
\lim _{n \rightarrow-\infty} \xi_{n}^{(i)}=y_{\ell_{i}}^{*}, \quad \lim _{n \rightarrow+\infty} \xi_{n}^{(i)}=y_{\ell_{i+1}}^{*}, \quad 1 \leqslant i \leqslant k
$$

Agora podemos provar o seguinte resultado relacionando $(G 1)$ e $(G 2)$ à não-existência de estruturas homoclínicas.

Lema 2.4.11. Seja $\left\{T^{n}: n \in \mathbb{N}\right\}$ uma semigrupo discreto que possui um número finito de soluções estacionárias $\mathcal{E}=\left\{y_{1}^{*}, \cdots y_{\mathfrak{p}}^{*}\right\}$ e um atrator global $\mathcal{A}$. Se $\left\{T^{n}: n \in \mathbb{N}\right\}$ satisfaz $(G 1)$, então (G2) é satisfeita se e somente se $\mathcal{A}$ não possui estruturas homoclínicas.

Demonstração: Assumindo que $\mathcal{A}$ tem uma estrutura homoclínica é fácil ver que as soluções estacionárias são recorrentes por cadeias.

Por outro lado, se $y^{*} \in \mathcal{E}$ é recorrente por cadeia, existe um $\delta<\delta_{0},\left\{y_{\ell_{1}}^{*}, \cdots, y_{\ell_{r+1}}^{*}\right\} \subset \mathcal{E}$, para cada $\mathbb{N} \ni k>\frac{1}{\delta}$, pontos $y_{1}^{k}, \cdots, y_{r+1}^{k}$ e números naturais $n_{1}^{k}>m_{1}^{k}, \cdots, n_{r}^{k}>m_{r}^{k}$ tais que

$$
d\left(y_{i}^{k}, y_{\ell_{i}}^{*}\right)<\frac{1}{k}, d\left(T^{m_{i}^{k}} y_{i}^{k}, \mathcal{E}\right)>\delta, d\left(T^{n_{i}^{k}} y_{i}^{k}, y_{\ell_{i+1}}^{*}\right)<\frac{1}{k}, 1 \leq i \leq r
$$

Escolha $\ell_{i}^{k}>0$ tal que $d\left(T^{\ell_{i}^{k}} y_{i}^{k}, y_{\ell_{i}}^{*}\right) \geq \delta$ e $d\left(T^{n} y_{i}^{k}, y_{\ell_{i}}^{*}\right)<\delta$, para todo $0 \leqslant n<\ell_{i}^{k}$. Do Lema 2.4.4 segue que $\ell_{i}^{k} \stackrel{k \rightarrow \infty}{\longrightarrow}+\infty$. Note que devemos ter também que $n_{i}^{k}-\ell_{i}^{k} \stackrel{k \rightarrow \infty}{\longrightarrow}+\infty$ (se não, existe $\nu \in \mathbb{N}$ tal que $n_{k}-\ell_{k}=\nu$ para infinitos índices $k$, consequentemente $\left\{T^{\ell_{k}} y_{i}^{k}: k \in \mathbb{N}\right\}$ tem uma subsequência convergente e portanto $\left\{T^{\nu} T^{\ell_{k}} y_{i}^{k}: k \in \mathbb{N}\right\}$ tem uma subsequência convergente para $y_{\ell_{i}}^{*}$, o que nos leva a uma contradição). Para $n \in\left[-\ell_{i}^{k}, n_{i}^{k}-\ell_{i}^{k}\right]$ seja $\xi_{n}^{i, k}=T_{i}^{\ell_{i}^{k}+n} y_{i}^{k}$.

Da Proposição 2.4.9, existem soluções globais $\xi_{i}: \mathbb{Z} \rightarrow X$ por $\xi_{n}^{(i)}=\lim _{k \rightarrow \infty} \xi_{n}^{i, k}$. Como cada $\xi_{n}^{(i)}$ deve convergir para uma solução de equilíbrio quando $n \rightarrow+\infty$ e quando $n \rightarrow-\infty$ e como $\xi_{n}^{(i)} \in B_{\delta}\left(y_{\ell_{i}}^{*}\right)$ para todo $n \leqslant 0$ temos que $\xi_{n}^{(i)} \rightarrow y_{\ell_{i}}^{*}$ quando $n \rightarrow-\infty$.

Além disso, existe um instante $j_{i}^{k}>0$ tal que $d\left(\xi_{j_{i}^{k}}^{i, k}, y_{\ell_{i+1}}^{*}\right) \geq \delta$ e $\xi_{n}^{i, k} \in B_{\delta}\left(y_{\ell_{i+1}}^{*}\right)$ para todo $n \in\left[j_{i}^{k}, n_{i}^{k}-\ell_{i}^{k}\right]$. É fácil ver que $\left\{j_{i}^{k}\right\}$ é limitado (Lema 2.4.5) e que $n_{i}^{k}-\ell_{i}^{k}-j_{i}^{k} \stackrel{k \rightarrow \infty}{\longrightarrow}$ $+\infty$. Consequentemente $\xi_{n}^{(i)} \rightarrow y_{\ell_{i+1}^{*}}^{*}$ quando $n \rightarrow+\infty$.

O conjunto $\left\{y_{\ell_{1}}^{*}, \cdots, y_{\ell_{k}}^{*}\right\} \subset \mathcal{E}$ e o conjunto de soluções globais $\left\{\xi_{i}: \mathbb{Z} \rightarrow X, 1 \leqslant i \leqslant k\right\}$ são tais que,

$$
\lim _{t \rightarrow-\infty} \xi_{n}^{(i)}=y_{\ell_{i}}^{*}, \quad \lim _{t \rightarrow+\infty} \xi_{n}^{(i)}=y_{\ell_{i+1}}^{*}, \quad 1 \leqslant i \leqslant k,
$$

$\operatorname{com} y_{\ell_{k+1}}^{*}:=y_{\ell_{1}}^{*}$. Portanto $\mathcal{A}$ tem uma estrutura homoclínica. 
Corolário 2.4.12. Se $\left\{T^{n}: n \in \mathbb{N}\right\}$ é um semigrupo discreto gradient-like e $\mathcal{A}$ seu atrator, existem pontos de equilíbrio $y_{\alpha}^{*}$ e $y_{\omega}^{*}$ tais que $y_{\alpha}^{*}$ tem conjunto estável trivial em $\mathcal{A} ;$ isto é, $W_{\mathcal{A}}^{s}\left(y_{\alpha}^{*}\right)=\left\{y_{\alpha}^{*}\right\}$ onde

$$
W_{\mathcal{A}}^{s}\left(y_{\alpha}^{*}\right):=\left\{y \in \mathcal{A}: \text { tal que } T^{n} y \stackrel{n \rightarrow \infty}{\longrightarrow} y_{\alpha}^{*}\right\}
$$

e $y_{\omega}^{*}$ tem conjunto instável trivial; isto é, $W^{u}\left(y_{\omega}^{*}\right)=\left\{y_{\omega}^{*}\right\}$.

Demonstração: Vamos provar a existência de um ponto de equilíbrio $y_{\omega}^{*}$ com um conjunto instável trivial. A existência de $y_{\alpha}^{*}$ é similar. Se $\mathcal{E}=\left\{y_{1}^{*}, \cdots y_{\mathfrak{p}}^{*}\right\}$ é o conjunto de equilíbrio para $\left\{T^{n}: n \in \mathbb{N}\right\}$ e para cada $y_{i}^{*}$ existe uma solução global $\xi^{(i)}: \mathbb{Z} \rightarrow X$ tal que $\xi^{(i)}(k) \stackrel{k \rightarrow-\infty}{\longrightarrow} y_{i}^{*}$, é fácil ver que existe um $1 \leqslant \ell \leqslant \mathfrak{p}$ tal que $\left\{y_{1}^{*}, \cdots, y_{\ell}^{*}\right\}$ e $\left\{\xi^{(1)}, \cdots, \xi^{(\ell)}\right\}$ constituem uma estrutura homoclínica e que contradiz $(G 2)$ e prova a existência de $y_{\omega}^{*}$.

A seguir consideremos uma família dependente de um parâmetro $\left\{T_{\eta}^{n}: n \in \mathbb{N}\right\}$, $\eta \in[0,1]$, de semigrupos discretos. Começamos com um resultado que estende o Lema 2.4 .4 para uma família $\left\{T_{\eta}^{n}: n \in \mathbb{N}\right\}, \eta \in[0,1]$, de semigrupos discretos.

Lema 2.4.13. Assuma que $\left\{T_{\eta}^{n}: n \in \mathbb{N}\right\}$ é tal que $T_{\eta} \stackrel{\eta \rightarrow 0}{\longrightarrow} T_{0}$ uniformemente em subconjuntos compactos de $X$. Seja $x^{*, \eta}$ um ponto fixo de $T_{\eta}, \eta \in[0,1]$ e assuma que $x^{*, \eta} \stackrel{\eta \rightarrow 0}{\longrightarrow} x^{*, 0}$. Dado $n \in \mathbb{N}$ e $\epsilon>0$, existe $\delta>0$ e $\eta_{0}>0$ tais que $\left\{T_{\eta}^{j} y: 0 \leqslant j \leqslant n, y \in B_{\delta}\left(x^{*, \eta}\right)\right\} \subset$ $B_{\epsilon}\left(x^{*, \eta}\right)$ para todo $\eta \leqslant \eta_{0}$.

Demonstração: Se este não é o caso existe uma sequência $\left\{\eta_{k}\right\}_{k \in \mathbb{N}} \operatorname{com} \eta_{k} \stackrel{k \rightarrow \infty}{\longrightarrow} 0, \epsilon_{0}>0$, $y_{k} \in B_{\frac{1}{k}}\left(x^{*, \eta_{k}}\right)$ e $n_{0} \in \mathbb{N}$ tal que $d\left(T_{\eta_{k}}^{n_{0}} y_{k}, x^{*, \eta_{k}}\right) \geqslant \epsilon_{0}$. Portanto $d\left(T_{0}^{n_{0}} x^{*, 0}, x^{*, 0}\right) \geqslant \epsilon_{0}$, o que é uma contradição.

Definição 2.4.14. Dizemos que $\left\{T_{\eta}^{n}: n \in \mathbb{N}\right\}$ é coletivamente assintoticamente compacto se, dadas uma sequência $\left\{\eta_{k}\right\}_{k \in \mathbb{N}}$ com $\eta_{k} \stackrel{k \rightarrow \infty}{\longrightarrow} 0$, uma sequência limitada $\left\{u_{k}\right\}_{k \in \mathbb{N}}$ em $X$ e uma sequência $\left\{n_{k}\right\}_{k \in \mathbb{N}}$ de números naturais com $n_{k} \stackrel{k \rightarrow \infty}{\longrightarrow} \infty$, então $\left\{T_{\eta_{k}}^{n_{k}} u_{k}\right\}$ é relativamente compacto.

Lema 2.4.15. Assuma que $\left\{T_{\eta}^{n}: n \in \mathbb{N}\right\}$ é coletivamente assintoticamente compacto. Seja $\eta_{k}$ uma sequência de números positivos tal que $\eta_{k} \stackrel{k \rightarrow \infty}{\longrightarrow} 0$. Assuma que exista uma sequência $\left\{j_{k}\right\}_{k \in \mathbb{N}}$ em $\mathbb{N}$ com $j_{k} \stackrel{k \rightarrow \infty}{\longrightarrow} \infty$ e seja $\mathbb{J}_{k}:=\left\{j \in \mathbb{Z}:|j| \leqslant j_{k}\right\}$. Se para cada 
$k \in \mathbb{N}$, existe uma solução $\xi_{\eta_{k}}(\cdot): \mathbb{J}_{k} \rightarrow X$ de $\left\{T_{\eta_{k}}^{n}: n \in \mathbb{N}\right\}$ e $\cup_{k \in \mathbb{N}} \xi_{\eta_{k}}\left(\mathbb{J}_{k}\right)$ é limitado então, existe uma subsequência, que novamente denotaremos por $\xi_{\eta_{k}}$, e uma solução global $y: \mathbb{Z} \rightarrow X$ de $\left\{T_{0}^{n}: n \in \mathbb{N}\right\}$ tal que

$$
\lim _{k \rightarrow \infty} \xi_{\eta_{k}}(j) \rightarrow y(j)
$$

para todo $j \in \mathbb{Z}$.

Demonstração: Como $\left\{T_{\eta}^{n}: n \in \mathbb{N}\right\}$ é coletivamente assintoticamente compacto, existe uma subsequência que novamente denotamos por $\eta_{k}$ e $y_{0} \in X$ tal que $\xi_{\eta_{k}}(0) \rightarrow y_{0}$. Seja $y(\cdot): \mathbb{N} \rightarrow X$ a solução de $\left\{T_{0}^{n}: n \in \mathbb{N}\right\}$ definida por $y(n):=T_{0}^{n} y_{0}, n \in \mathbb{N}$. Claramente, esta solução é limitada. Também

$$
\xi_{\eta_{k}}(j)=T_{\eta_{k}}^{j} \xi_{\eta_{k}}(0) \stackrel{k \rightarrow \infty}{\longrightarrow} T_{0}^{j} y_{0}=y(j), \quad \forall j \in \mathbb{N} .
$$

Procedendo similarmente, $\left\{\xi_{\eta_{k}}(-1)\right\}$ tem uma subsequência convergente $\xi_{\eta_{k}^{1}}(-1)$ com limite $y_{-1}$. Definindo $y(j):=T_{0}^{j+1} y_{-1}, j \in \mathbb{Z}_{-1}^{+}$, temos que $y(0)=T_{0} y_{-1}=\lim _{k \rightarrow \infty} T_{\eta_{k}^{1}} \xi_{\eta_{k}^{1}}(-1)=$ $\lim _{k \rightarrow \infty} \xi_{\eta_{k}^{1}}(0)=y_{0}$ e $y(j)=T_{0}^{j+1} y_{-1}$ para todo $j \geqslant-1$. Disto segue que $y(\cdot): \mathbb{Z}_{-1}^{+} \rightarrow X$ é uma solução de $\left\{T_{0}^{n}: n \in \mathbb{N}\right\}$ com $y(-1)=y_{-1}, y(0)=y_{0} \mathrm{e}$

$$
\xi_{\eta_{k}^{1}}(j)=T_{\eta_{k}}^{j+1} \xi_{\eta_{k}^{1}}(-1) \rightarrow T_{0}^{j+1} y_{-1}=y(j), \quad j \in \mathbb{Z}_{-1}^{+}
$$

Suponha que:

- Tenhamos obtido subsequências $\left\{\xi_{\eta_{k}^{i}}\right\}_{k \in \mathbb{N}}$ para $1 \leqslant i \leqslant m-1$ com a propriedade de que $\left\{\eta_{k}^{i}\right\}_{k \in \mathbb{N}}$ é uma subsequência de $\left\{\eta_{k}^{i-1}\right\}_{k \in \mathbb{N}}$ e tal que $\xi_{\eta_{k}^{i}}(-i) \rightarrow y_{-i}, 1 \leqslant i \leqslant m-1$.

- Tenhamos definido $y(j)$ por $\lim _{n \rightarrow \infty} \xi_{\eta_{k}^{i}}(j)=y(j),-m+1 \leqslant j<0$ e, consequentemente, $y: \mathbb{Z}_{-m+1}^{+} \rightarrow X$ é uma solução de $\left\{T_{0}^{n}: n \in \mathbb{N}\right\}$ com $y(-j)=y_{-j}$, $0 \leqslant j \leqslant-m+1$ e $\xi_{\eta_{k}^{i}}(j)$ converge para $y(j)$ para todo $j \in \mathbb{Z}_{m-1}^{+}$.

Agora construímos $\left\{\eta_{k}^{m}\right\}_{n=1}^{\infty}$ uma subsequência de $\left\{\eta_{k}^{m-1}\right\}_{n=1}^{\infty}$ tal que $\xi_{\eta_{k}^{m}}(-m)$ é convergente e $y_{-m}$ seu limite. Se nós definirmos $y(-m)=y_{-m}, y: \mathbb{Z}_{-m}^{+} \rightarrow X$ é uma solução de $\left\{T_{0}^{n}: n \in \mathbb{N}\right\}$ com $y(-i)=y_{-i}, 0 \leqslant i \leqslant m$ e $\xi_{\eta_{k}^{m}}(j)$ converge para $y(j)$ para todo $j \in \mathbb{Z}_{-m}^{+}$.

Com isto construímos uma sequência $\left\{\xi_{\eta_{k}^{k}}\right\}_{k=1}^{\infty}$ e uma solução $y(\cdot): \mathbb{Z} \rightarrow X$ de $\left\{T_{0}^{n}:\right.$ 
$n \in \mathbb{N}\}$ com $y(-i)=y_{-i}$ para todo $i \in \mathbb{N}$ e tal que $\xi_{\eta_{k}^{k}}(j) \rightarrow y(j)$ para todo $j \in \mathbb{Z}$. Isto conclui a demonstração.

Corolário 2.4.16. Assuma que $\left\{T_{\eta}^{n}: n \in \mathbb{N}\right\}$ é coletivamente assintoticamente compacto. Seja $\left\{\eta_{k}\right\}_{k \in \mathbb{N}}$ uma sequência em $(0,1]$ tal que $\eta_{k} \stackrel{k \rightarrow \infty}{\longrightarrow} 0$ e para cada $k \in \mathbb{N}$ seja $\xi_{\eta_{k}}(\cdot): \mathbb{Z} \rightarrow$ $X$ uma solução global de $\left\{T_{\eta_{k}}^{n}: n \in \mathbb{N}\right\}$. Se $\cup_{k \in \mathbb{N}} \xi_{\eta_{k}}(\mathbb{Z})$ é limitado, para qualquer sequência $\mathbf{s}=\left\{s_{k}\right\}_{k \in \mathbb{N}}$ em $\mathbb{Z},\left\{\xi_{\eta_{k}}\right\}_{k \in \mathbb{N}}$ tem uma subsequência que denotaremos por $\left\{\xi_{\eta_{k}^{s}}\right\}_{k \in \mathbb{N}}$ e uma solução global $y^{\mathbf{s}}: \mathbb{Z} \rightarrow X$ de $\left\{T_{0}^{n}: n \in \mathbb{N}\right\}$ tal que

$$
\lim _{k \rightarrow \infty} \xi_{\eta_{k}^{\mathbf{s}}}\left(j+s_{k}\right) \rightarrow y^{\mathbf{s}}(j)
$$

para todo $j \in \mathbb{Z}$.

Lema 2.4.17. Seja $\left\{T_{\eta}^{n}: n \in \mathbb{N}\right\}, \eta \in[0,1]$, uma família de semigrupos discretos coletivamente assintoticamente compactos. Assuma que $\left\{T_{\eta}^{n}: n \in \mathbb{N}\right\}$ tem um atrator global $\mathcal{A}_{\eta}$ e $\mathfrak{p}$ soluções estacionárias com $\mathfrak{p}$ independente de $\eta$. Denote por $\mathcal{E}_{\eta}=\left\{y_{1}^{*, \eta}, \cdots y_{\mathfrak{p}}^{*, \eta}\right\}$ o conjunto de soluções estacionárias de $\left\{T_{\eta}^{n}: n \in \mathbb{N}\right\}$ e defina $\mathcal{E}_{0}=\left\{y_{1}^{*}, \cdots y_{\mathfrak{p}}^{*}\right\}$. Se $d\left(T_{\eta} u, T_{0} u\right) \stackrel{\eta \rightarrow 0}{\longrightarrow} 0$ uniformemente para $u$ em subconjuntos compacto de $X$ e $\left\{T_{0}^{n}: n \in \mathbb{N}\right\}$ é gradient-like, $\delta<\delta_{0}$ e $B \subset X$ é limitado, existe um $n_{0}=n_{0}(\delta, B)>0$, independente de $\eta \in[0,1]$, tal que $\left\{T_{\eta}^{n} u_{0}: 0 \leqslant n \leqslant n_{0}\right\} \cap \cup_{i=1}^{\mathfrak{p}} B_{\delta}\left(y_{i}^{*}\right) \neq \emptyset$ para todo $u_{0} \in B$.

Demonstração: Vamos fazer uma argumentação por contradição. Assuma que existe uma sequência $\left\{u_{k}\right\}_{k \in \mathbb{N}}$ em $B$, uma sequência $\left\{\eta_{k}\right\}_{k \in \mathbb{N}}$ em $[0,1]$ com $\eta_{k} \stackrel{k \rightarrow \infty}{\longrightarrow} 0$ e uma sequência $\left\{n_{k}\right\}_{k \in \mathbb{N}}$ em $\mathbb{N}$ com $n_{k} \stackrel{k \rightarrow \infty}{\longrightarrow} \infty$ tais que $\left\{T_{\eta_{k}}^{j} u_{k}: 0 \leqslant j \leqslant 2 n_{k}\right\} \cap \cup_{i=1}^{\mathfrak{p}} B_{\delta}\left(y_{i}^{*}\right)=\emptyset$. Tomando subsequência temos que existe $v_{0} \in X$ tal que $T_{\eta_{k}}^{n_{k}} u_{k} \stackrel{k \rightarrow \infty}{\longrightarrow} v_{0}$. Do fato de que $T_{\eta}$ converge para $T_{0}$ uniformemente em subconjuntos compactos de $X$ temos que $T_{\eta_{k}}^{r+n_{k}} u_{k} \stackrel{k \rightarrow \infty}{\longrightarrow} T_{0}^{r} v_{0}$ para todo $r \in \mathbb{N}$. Do Lema 2.4.7 existe um $r_{0}$ e $y_{j}^{*} \in \mathcal{E}$ tal que $d\left(T_{0}^{r} v_{0}, y_{j}^{*}\right)<\delta$ para todo $r \geqslant r_{0}$ a assim chegamos a uma contradição.

Lema 2.4.18. Seja $\left\{T_{\eta}^{n}: n \in \mathbb{N}\right\}, \eta \in[0,1]$, uma família de semigrupos discretos coletivamente assintoticamente compactos. Assuma que $\left\{T_{\eta}^{n}: n \in \mathbb{N}\right\}$ tem um atrator global $\mathcal{A}_{\eta}$ e $\mathfrak{p}$ soluções estacionárias com $\mathfrak{p}$ independente de $\eta$. Denote por $\mathcal{E}_{\eta}=\left\{y_{1}^{*, \eta}, \cdots y_{\mathfrak{p}}^{*, \eta}\right\}$ o conjunto das soluções estacionárias de $\left\{T_{\eta}^{n}: n \in \mathbb{N}\right\}$ e defina $\mathcal{E}_{0}=\left\{y_{1}^{*}, \cdots y_{\mathfrak{p}}^{*}\right\}$. Dado $0<\delta<\delta_{0}$, existe um $\eta_{0}>0$ e $\delta^{\prime}>0$ tal que, se para algum $1 \leqslant i \leqslant \mathfrak{p}, d\left(u_{0}, y_{i}^{*}\right)<\delta^{\prime}$ e, 
para algum $n_{1}>0, d\left(T_{\eta}^{n_{1}} u_{0}, y_{i}^{*}\right) \geqslant \delta$, então $d\left(T_{\eta}^{n} u_{0}, y_{i}^{*}\right)>\delta^{\prime}$ para todo $n \geqslant n_{1}$ e para todo $\eta \in\left[0, \eta_{0}\right]$.

Demonstração: Assuma que, para algum $1 \leqslant i \leqslant \mathfrak{p}$, existe uma sequência $u_{k}$ em $X$, $\left\{\eta_{k}\right\}_{k \in N}$ com $\eta_{k} \stackrel{k \rightarrow \infty}{\longrightarrow} 0$, com $d\left(u_{k}, y_{i}^{*}\right)<\frac{1}{k}$ e sequências $n_{k}<m_{k}$ de números naturais tais que $d\left(T_{\eta_{k}}^{n_{k}} u_{k}, y_{i}^{*}\right) \geqslant \delta$ e $d\left(T_{\eta_{k}}^{m_{k}} u_{k}, y_{i}^{*}\right)<\frac{1}{k}$. Mostremos que isto contradiz $(G 2)$.

Note primeiramente que $n_{k}$ podem ser escolhidos de forma que $T_{\eta_{k}}^{j} u_{k} \in B_{\delta}\left(y_{i}^{*}\right)$ para todo $j<n_{k}$ e, do Lema 2.4.13, $n_{k} \stackrel{k \rightarrow \infty}{\longrightarrow} \infty$. Tomando subsequências construímos uma solução global $\xi_{0}: \mathbb{Z} \rightarrow X$ in $\mathcal{A}$ tal que $\xi_{0}(\ell) \stackrel{\ell \rightarrow-\infty}{\longrightarrow} y_{i}^{*}$. Do fato de que $\left\{T_{0}^{n}: n \in \mathbb{N}\right\}$ é gradient-like temos que existe um $y_{j}^{*} \in \mathcal{E}, y_{j}^{*} \neq y_{i}^{*}$, tal que $\xi_{0}(\ell) \stackrel{\ell \rightarrow \infty}{\longrightarrow} y_{j}^{*}$.

Da definição de $\xi_{0}$ e do fato de que $\xi_{0}(\ell) \stackrel{\ell \rightarrow \infty}{\longrightarrow} y_{j}^{*}$ temos que para cada $k^{\prime} \in \mathbb{N}$ existe um $u_{k^{\prime}} \in \phi_{0}(\mathbb{Z})$, com $d\left(u_{k^{\prime}}, y_{j}^{*}\right)<\frac{1}{k^{\prime}}$ e $n_{k^{\prime}} \in \mathbb{N}$ tal que $d\left(T_{\eta_{k^{\prime}}}^{n_{k^{\prime}}} u_{k^{\prime}}, y_{j}^{*}\right) \geqslant \delta$. Procedendo exatamente como no passo anterior obtemos uma solução $\xi_{1}: \mathbb{Z} \rightarrow X$ in $\mathcal{A}$ tal que $\xi_{1}(k) \stackrel{k \rightarrow-\infty}{\longrightarrow} y_{j}^{*}$. Do fato de que $\left\{T_{0}^{n}: n \in \mathbb{N}\right\}$ é gradient-like temos que existem $y_{r}^{*} \in \mathcal{E}$, $y_{r}^{*} \notin\left\{y_{i}^{*}, y_{j}^{*}\right\}$, tais que $\xi_{1}(\ell) \stackrel{\ell \rightarrow \infty}{\longrightarrow} y_{r}^{*}$. Continuando com este procedimento chegamos em uma contradição em um número finito de passos.

A maior vantagem dos semigrupos gradient-like sobre os semigrupos gradientes (aqueles com uma função de Liapunov) é a sua estabilidade por perturbações, que exploraremos a seguir. Este fato possibilitará a caracterização de atratores de semigrupos discretos que são pequenas perturbações de semigrupos discretos gradient-like, perturbar tais semigrupos novamente e ainda seremos capazes de dar uma caracterização dos atratores deste novo semigrupo discreto perturbado.

Agora estamos prontos para provar que $(G 1)$ e $(G 2)$ são estáveis por perturbações; isto é, o conceito de semigrupo discreto gradient-like é robusto sob perturbações.

Teorema 2.4.19. Seja $\left\{T_{\eta}^{n}: n \in \mathbb{N}\right\}, \eta \in[0,1]$, uma família de semigrupos discretos coletivamente assintoticamente compacto em um espaço métrico $X$ que satisfaz

a) para cada $\eta \in[0,1]\left\{T_{\eta}^{n}: n \in \mathbb{N}\right\}$ tem um atrator global $\mathcal{A}_{\eta}$.

b) $\left\{T_{\eta}^{n}: n \in \mathbb{N}\right\}$ tem um número finito de soluções estacionárias $\mathcal{E}_{\eta}=\left\{y_{1}^{*, \eta}, \cdots, y_{\mathfrak{p}}^{*, \eta}\right\}$, para todo $\eta \in[0,1]$, e $d\left(y_{i}^{*, \eta}, y_{i}^{*, 0}\right) \stackrel{\eta \rightarrow 0}{\longrightarrow} 0,1 \leqslant i \leqslant \mathfrak{p}$. Em adição, existem $\delta>0$ e $\eta_{0}>0$ tais que a única solução global contida em $B_{\delta}\left(y_{i}^{*, \eta}\right)$ é $y_{i}^{*, \eta}, 1 \leqslant i \leqslant \mathfrak{p}$, $0 \leqslant \eta \leqslant \eta_{0}$. 
c) existe $\delta>0$ tal que, se uma solução $\xi^{(\eta)}: \mathbb{Z} \rightarrow X$ em $\mathcal{A}_{\eta}$ é tal que $d\left(\xi_{n}^{(\eta)}, y_{i}^{*, \eta}\right) \leq \delta$, para todo $n \geqslant n_{0}$ (ou para todo $\left.n \leqslant n_{0}\right), n_{0} \in \mathbb{Z}$, então $\xi_{n}^{(\eta)} \stackrel{n \rightarrow \infty}{\longrightarrow} y_{i}^{*, \eta}\left(\right.$ ou $\xi_{n}^{(\eta)} \stackrel{n \rightarrow-\infty}{\longrightarrow}$ $\left.y_{i}^{*, \eta}\right)$.

d) $d\left(T_{\eta} u, T_{0} u\right) \stackrel{\eta \rightarrow 0}{\longrightarrow} 0$ uniformemente para $u$ em subconjuntos compactos de $X$.

e) $\left\{T_{0}^{n}: n \in \mathbb{N}\right\}$ é um semigrupo discreto gradient-like.

Então, existe $\eta_{0}>0$ tal que, para todo $\eta \in\left[0, \eta_{0}\right]$, o semigrupo discreto $\left\{T_{\eta}^{n}: n \in \mathbb{N}\right\}$ é gradient-like.

Demonstração: Usaremos a contradição para provar que para $\eta$ suficientemente pequeno, $\left\{T_{\eta}^{n}: n \in \mathbb{N}\right\}$ satifaz que, dada uma solução global $\xi^{(\eta)}: \mathbb{Z} \rightarrow X$ em $\mathcal{A}_{\eta}$,

$$
\lim _{n \rightarrow \infty} d\left(\xi_{n}^{(\eta)}, y_{i}^{*, \eta}\right)=0, \text { para algum } 1 \leqslant i \leqslant \mathfrak{p}
$$

Note que $y_{i}^{*, \eta} \rightarrow y_{i}^{*, 0}=: y_{i}^{*}$ e que existe um $\delta>0$ tal que, para $\eta$ suficientemente pequeno, se uma solução $\xi^{(\eta)}: \mathbb{Z} \rightarrow X$ satisfaz $d\left(\xi_{n}^{(\eta)}, y_{i}^{*}\right) \leq \delta$ para todo $n \geqslant n_{0}$ e para algum $n_{0} \in \mathbb{Z}$, então $\xi_{n}^{(\eta)} \stackrel{n \rightarrow \infty}{\longrightarrow} y_{i}^{*, \eta}$. Assuma que existe uma sequência $\eta_{k} \stackrel{k \rightarrow \infty}{\longrightarrow} 0$ e soluções globais correspondentes $\xi^{(k)}$ em $\mathcal{A}_{\eta_{k}}$ tais que

$$
\sup _{n \geqslant j} \operatorname{dist}\left(\xi_{n}^{(k)}, \mathcal{E}\right)>\delta, \forall j \in \mathbb{N}
$$

para cada $k \in \mathbb{N}$. Pelo Corolário 2.4.16 tomando subsequências, $\xi_{n}^{(k)} \stackrel{k \rightarrow \infty}{\longrightarrow} \xi_{n}^{(0)}$ para cada $n \in \mathbb{Z}$ onde $\xi^{(0)}$ é uma solução global em $\mathcal{A}_{0}$. Como $\xi_{n}^{(0)} \stackrel{n \rightarrow \infty}{\longrightarrow} y_{i}^{*}$, para algum $1 \leqslant i \leqslant \mathfrak{p}$, temos que, dado $r \in \mathbb{N} \backslash\{0\}$ existem $n_{r}>0$ e $k_{r} \in \mathbb{N}$ tais que $d\left(\xi_{n_{r}}^{(k)}, y_{i}^{*}\right)<\frac{1}{r}$, para cada $k \geqslant k_{r}$. De (2.4.1), existe $n_{r}^{\prime}>n_{r}$ tal que $d\left(\xi_{n}^{\left(k_{r}\right)}, y_{i}^{*}\right)<\delta$ para todo $n \in\left[n_{r}, n_{r}^{\prime}\right)$ e $d\left(\xi_{n_{r}^{\prime}}^{\left(k_{r}\right)}, y_{i}^{*}\right) \geqslant \delta$.

Tomando subsequências se necessário, seja $\xi_{n}^{(1)}=\lim _{r \rightarrow \infty} \xi_{n+n_{r}^{\prime}}^{\left(k_{r}\right)}$. Então, como $n_{r}^{\prime}-$ $n_{r} \stackrel{r \rightarrow \infty}{\longrightarrow} \infty$ e $n_{r} \stackrel{r \rightarrow \infty}{\longrightarrow}+\infty, d\left(\xi_{n}^{(1)}, y_{i}^{*}\right) \leqslant \delta$ para todo $n<0$ e consequentemente $\xi_{n}^{(1)} \stackrel{n \rightarrow-\infty}{\longrightarrow} y_{i}^{*}$.

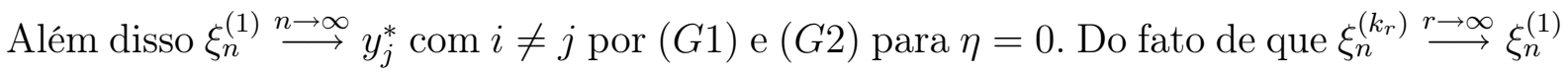
para todo $n \in \mathbb{Z}$ temos que, para cada $m \in \mathbb{N}$, existe um instante $n_{m}>0$ e índices $k_{m} \in \mathbb{N}$ tais que $d\left(\xi_{n_{m}}^{\left(k_{r}\right)}, y_{j}^{*}\right)<\frac{1}{m}$ para todo $r \geqslant k_{m}$. Novamente, de 2.4.1), existem $n_{m}^{\prime}>n_{m}$ tais que $d\left(\xi_{n}^{k_{m}}, y_{j}^{*}\right)<\delta$ para todo $n \in\left[n_{m}, n_{m}^{\prime}\right)$ e $d\left(\xi_{n_{m}^{\prime}}^{k_{m}}, y_{j}^{*}\right) \geqslant \delta$. Procedendo exatamente como antes obtemos uma solução global $\xi^{(2)}: \mathbb{Z} \rightarrow X$ tal que $\xi_{n}^{(2)} \stackrel{n \rightarrow-\infty}{\longrightarrow} y_{j}^{*}$ e $\xi_{n}^{(2) ~} \stackrel{n \rightarrow \infty}{\longrightarrow} y_{\ell}^{*}$ 
com $\ell \notin\{i, j\}$. Em um número finito de passos, atingimos uma contradição. Isto prova que existe um $\eta_{0}>0$ tal que, para toda solução global $\xi^{(\eta)}$ em $\mathcal{A}_{\eta} \operatorname{com} \eta \leqslant \eta_{0}$, temos que

$$
\lim _{n \rightarrow \infty} d\left(\xi_{n}^{(\eta)}, y_{i}^{*, \eta}\right)=0
$$

Para provar que existe um $\eta_{1}>0$ tal que, para toda solução global $\xi^{(\eta)}$ em $\mathcal{A}_{\eta}$ com $\eta \leqslant \eta_{1}$, temos que

$$
\lim _{n \rightarrow-\infty} d\left(\xi_{n}^{(\eta)}, y_{j}^{*, \eta}\right)=0
$$

procedemos exatamente da mesma maneira. Isto completa a prova de que, para $\eta$ suficientemente pequeno, $\left\{T_{\eta}^{n}: n \in \mathbb{N}\right\}$ satisfaz $(G 1)$.

Vamos provar que, para $\eta$ suficientemente pequeno, $\left\{T_{\eta}^{n}: n \in \mathbb{N}\right\}$ satisfaz (G2). Novamente argumentaremos por contradição. Assuma que existe uma sequência $y_{1}^{*}, \cdots, y_{q+1}^{*}$ em $\mathcal{E}$, um $0<\delta<\delta_{0}$, uma sequência $\eta_{k} \rightarrow 0$, soluções globais $\xi^{k, i}$ em $\mathcal{A}_{\eta_{k}}$, e tempos $n_{1}^{k}>m_{1}^{k}, \cdots, n_{q}^{k}>m_{q}^{k}$ tais que

$$
d\left(\xi_{0}^{k, i}, y_{i}^{*}\right)<\frac{1}{k}, \quad d\left(\xi_{m_{i}^{k}}^{k, i}, \mathcal{E}\right) \geqslant \delta, \quad d\left(\xi_{n_{i}^{k}}^{k, i}, y_{i+1}^{*}\right)<\frac{1}{k}, \quad 1 \leqslant i \leqslant q, \quad y_{1}^{*}=y_{q+1}^{*}
$$

Procedendo como na prova de $(G 1)$ construímos uma estrutura homoclínica para $\left\{T_{0}^{n}\right.$ : $n \in \mathbb{N}\}$ e chegamos a uma contradição.

Como consequência imediata deste teorema nós obtemos o seguinte resultado de caracterização.

Corolário 2.4.20. Sob as hipóteses do Teorema 2.4.19, existe um $\eta_{0}>0$ tal que

$$
\mathcal{A}_{\eta}=\cup_{i=1}^{\mathfrak{p}} W^{u}\left(y_{i}^{*, \eta}\right), \forall \eta \in\left[0, \eta_{0}\right]
$$

Agora vamos substituir o conjunto de equilíbrio finito por uma coleção finita de conjuntos invariantes isolados. A definição de semigrupos discretos gradient-like será alterada de acordo e os resultados análogos possuem provas similares.

Definição 2.4.21. Dizemos $\mathcal{S}=\left\{\Xi_{1}^{*}, \cdots \Xi_{\mathfrak{p}}^{*}\right\}$ é uma familia de conjuntos invariantes isolados se existe $\delta>0$ tal que $\mathcal{O}_{\delta}\left(\Xi_{i}^{*}\right) \cap \mathcal{O}_{\delta}\left(\Xi_{j}^{*}\right)=\emptyset, 1 \leqslant i<j \leqslant \mathfrak{p}$, e $\Xi_{i}^{*}$ é o subconjunto invariante maximal de $\mathcal{O}_{\delta}\left(\Xi_{i}^{*}\right):=\left\{z \in X: \operatorname{dist}\left(z, \Xi_{i}^{*}\right)<\delta\right\}$.

Seja $\left\{T^{n}: n \in \mathbb{N}\right\}$ um semigrupo discreto com um atrator global $\mathcal{A}$ que contém uma família finita de conjuntos invariantes isolados $\mathcal{S}=\left\{\Xi_{1}^{*}, \cdots \Xi_{\mathfrak{p}}^{*}\right\}$. Definimos 
Definição 2.4.22. Seja $\delta$ como na Definição 2.4.21 e fixe $\epsilon_{0} \in(0, \delta)$. Para $\Xi^{*} \in \mathcal{S}$ e $\epsilon \in\left(0, \epsilon_{0}\right)$, uma $\epsilon$-cadeia e $\Xi^{*}$ a $\Xi^{*}$ é uma sequência $\left\{\Xi_{\ell_{i}}^{*}, \cdots, \Xi_{\ell_{k}}^{*}\right\} \subset \mathcal{S}$, uma sequência de números reais $n_{1}, \kappa_{1}, \cdots, n_{k}, \kappa_{k}$, com $n_{i}>\kappa_{i}, 1 \leqslant i \leqslant k, k \leqslant \mathfrak{p}$, e uma sequência de vetores $u_{i}, 1 \leqslant i \leqslant k$, tais que $u_{i} \in \mathcal{O}_{\epsilon}\left(\Xi_{\ell_{i}}^{*}\right), T^{\kappa_{i}} u_{i} \notin \mathcal{O}_{\epsilon_{0}}\left(\cup_{i=1}^{k}\left(\Xi_{\ell_{i}}^{*}\right)\right)$ e $T^{n_{i}} u_{i} \in \mathcal{O}_{\epsilon}\left(\Xi_{\ell_{i+1}}^{*}\right)$, $1 \leqslant i \leqslant k$, com $\Xi^{*}=\Xi_{\ell_{k+1}}^{*}=\Xi_{\ell_{1}}^{*}$. Dizemos que $\Xi^{*} \in \mathcal{S}$ é recorrente por cadeias se existe $u m \epsilon_{0} \in(0, \delta)$ e $\epsilon$-cadeias de $\Xi^{*} a \Xi^{*}$ para cada $\epsilon \in\left(0, \epsilon_{0}\right)$.

Definição 2.4.23. Seja $\left\{T^{n}: n \in \mathbb{N}\right\}$ um semigrupo discreto em um espaço métrico $X$. Seja $\mathcal{A}$ o atrator global para $\left\{T^{n}: n \in \mathbb{N}\right\}$. Dizemos que $\left\{T^{n}: n \in \mathbb{N}\right\}$ é um semigrupo gradient-like generalizado se existe uma família finita $\mathcal{S}=\left\{\Xi_{1}^{*}, \cdots, \Xi_{\mathfrak{p}}^{*}\right\}$ de conjuntos invariantes isolados em $\mathcal{A}$ tal que,

(GG1) Para cada solução global $\xi: \mathbb{Z} \rightarrow X$ em $\mathcal{A}$ existem $1 \leqslant i, j \leqslant \mathfrak{p}$ tais que

$$
\lim _{n \rightarrow-\infty} \operatorname{dist}\left(\xi_{n}, \Xi_{i}^{*}\right)=0 \text { e } \lim _{n \rightarrow \infty} \operatorname{dist}\left(\xi_{n}, \Xi_{j}^{*}\right)=0 .
$$

(GG2) Nenhum elemento de $\mathcal{S}=\left\{\Xi_{1}^{*}, \cdots, \Xi_{\mathfrak{p}}^{*}\right\}$ é recorrente por cadeias.

Como anteriormente introduzimos as definições de conjuntos instáveis e estáveis.

Definição 2.4.24. Seja $\left\{T^{n}: n \in \mathbb{N}\right\}$ um semigrupo discreto. O conjunto instável de um conjunto invariante isolado $\Xi^{*}$ é dada por

$$
\begin{gathered}
W^{\mathrm{u}}\left(\Xi^{*}\right)=\{\zeta \in X \text { : existe uma solução global } \xi: \mathbb{Z} \rightarrow X \\
\text { tal que } \left.\xi_{0}=\zeta \text { e } \lim _{n \rightarrow-\infty} \operatorname{dist}\left(\xi_{n}, \Xi^{*}\right)=0\right\} .
\end{gathered}
$$

$O$ conjunto estável de um conjunto invariante isolado $\Xi^{*}$ para $\left\{T^{n}: n \in \mathbb{N}\right\}$ é dada por

$$
W^{\mathrm{s}}\left(\Xi_{\eta}^{*}\right)=\left\{\zeta \in X: \lim _{n \rightarrow+\infty} \operatorname{dist}\left(T^{n} \zeta, \Xi^{*}\right)=0\right\}
$$

A interseção de conjuntos instáveis (estáveis) com uma vizinhança de $\Xi^{*}$ em $X$ é chamado de conjunto instável (estável) local e é denotado por $W_{\eta, \text { loc }}^{\mathrm{u}}\left(W_{\eta, \mathrm{loc}}^{\mathrm{s}}\right)$.

A demonstração do seguinte resultado é completamente análoga à do Teorema 2.4.19. 
Teorema 2.4.25. Seja $\left\{T_{\eta}^{n}: n \in \mathbb{N}\right\}, \eta \in[0,1]$, uma família de semigrupos discretos coletivamente assintoticamente compactos em um espaço métrico X. Assuma que

a) $\left\{T_{\eta}^{n}: n \in \mathbb{N}\right\}$ tem atrator global $\mathcal{A}_{\eta}, \eta \in[0,1]$.

b) $\mathcal{A}_{\eta}$ tem um número finito de conjuntos invariantes isolados $\mathcal{S}_{\eta}=\left\{\Xi_{1, \eta}^{*}, \cdots, \Xi_{\mathfrak{p}, \eta}^{*}\right\}$, $\eta \in[0,1]$, que se comportam semicontinuamente superiormente e inferiormente $\eta \rightarrow 0\left(\sup _{1 \leqslant i \leqslant p}\left[\operatorname{dist}_{H}\left(\Xi_{i, \eta}^{*}, \Xi_{i, 0}^{*}\right)+\operatorname{dist}_{H}\left(\Xi_{i, 0}^{*}, \Xi_{i, \eta}^{*}\right)\right] \stackrel{\eta \rightarrow 0}{\longrightarrow} 0\right)$. Em adição, existem $\delta>0$ e $\eta_{0}>0$ tais que as únicas soluções globais contidas em $B_{\delta}\left(\Xi_{i, \eta}^{*}\right)$ estão em $\Xi_{i, \eta}^{*}, 1 \leqslant i \leqslant \mathfrak{p}, 0 \leqslant \eta \leqslant \eta_{0}$.

c) $d\left(T_{\eta}^{n} u, T_{0}^{n} u\right) \stackrel{\eta \rightarrow 0}{\longrightarrow} 0$ para cada $n \in \mathbb{N}$ e uniformemente para $u$ em subconjuntos compacto de $X$.

d) existem $\delta>0$ e $\eta_{0} \in(0,1]$ tais que, se $\eta<\eta_{0}, \xi^{(\eta)}: \mathbb{Z} \rightarrow X$ é uma solução global em $\mathcal{A}_{\eta}$, e $\operatorname{dist}\left(\xi_{n}^{(\eta)}, \Xi_{i, \eta}^{*}\right)<\delta$ para todo $n \leqslant 0(n \in \mathbb{N})$, então $\operatorname{dist}\left(\xi_{n}^{(\eta)}, \Xi_{i}^{*}\right) \stackrel{n \rightarrow-\infty}{\longrightarrow} 0$ $\left(\operatorname{dist}\left(\xi_{n}^{(\eta)}, \Xi_{i}^{*} \stackrel{t \rightarrow+\infty}{\longrightarrow} 0\right)\right.$

e) $\left\{T_{0}^{n}: n \in \mathbb{N}\right\}$ é um semigrupo discreto gradient-like generalizado.

Então existe $\eta_{0}>0$ tal que, para todo $\eta \leqslant \eta_{0},\left\{T_{\eta}^{n}: n \in \mathbb{N}\right\}$ é um semigrupo discreto gradient-like generalizado. Consequentemente, existe $\eta_{0}>0$ tal que

$$
\mathcal{A}_{\eta}=\cup_{i=1}^{\mathfrak{p}} W^{u}\left(\Xi_{i, \eta}^{*}\right), \forall \eta \in\left[0, \eta_{0}\right]
$$

Isto abre possibilidades para considerarmos perturbações não-autônomas de semigrupos não-lineares gradient-like generalizados com soluções periódicas. De fato, para cada $1 \leq m \in \mathbb{N}$, a aplicação no tempo um para o problema

$$
\begin{aligned}
& \dot{r}= \begin{cases}\pi^{-1}\left(1-\frac{1}{2 m+1}-r\right)^{3} \operatorname{sen} \frac{\pi}{1-r}, & r<1-\frac{1}{2 m+1} \\
-\left(1-\frac{1}{2 m+1}-r\right)^{2}, & r \geq 1-\frac{1}{2 m+1}\end{cases} \\
& \dot{\theta}=\pi
\end{aligned}
$$

tem atrator $\mathcal{A}_{m}=\left\{|r| \leq 1-\frac{1}{2 m+1}\right\}$, que é a união das variedades instáveis de $\Xi_{j}^{*}, 1 \leq$ $j \leq 2 m+1$, onde $\Xi_{j}^{*}$ é a solução 2 -periódica correspondente à $r=1-\frac{1}{j}, 1 \leq j \leq 2 m+2$. Estas soluções periódicas são normalmente soluções hiperbólicas (se $k$ é par, a órbita é 
instável, e se $k$ é ímpar, a órbita é estável). Neste caso, é fácil ver que o atrator $\mathcal{A}_{m}$ é a união das variedades instáveis das soluções periódicas $\left\{\Xi_{j}^{*}: 1 \leq j \leq 2 m+1\right\}$. $\mathrm{O}$ Teorema 2.4.25 implica que qualquer perturbação pequena da semigrupo discreto dado pela aplicação no tempo um associado à (2.4.2 nos levará a um processo de evolução gradient-like generalizado e o atrator perturbado é caracterizado.

\subsection{Atratores exponenciais}

Seja $\left\{T^{n}: n \in \mathbb{N}\right\}$ um semigrupo discreto não-linear que tem um atrator global $\mathcal{A}$. Dizemos que $\mathcal{A}$ é um atrator exponencial se existem $\varrho>0$ e para cada conjunto limitado $B$ uma constante positiva $C(B)$ tal que

$$
\operatorname{dist}_{H}\left(T^{n} B, \mathcal{A}\right) \leqslant C(B) e^{-\varrho n}, n \in \mathbb{N}
$$

Mostraremos agora, seguindo Babin-Vishik [1, que o atrator global de um semigrupo discreto gradient-like que tem somente um número finito de soluções estacionárias, cada uma delas atraindo exponencialmente conjuntos instáveis locais é também um atrator exponencial.

Suponha que $\left\{T^{n}: n \in \mathbb{N}\right\}$ satisfaz que, para cada conjunto limitado $B$, existem $c=c(B)$ e $L>0$ tais que

$$
d\left(T^{n} u, T^{n} v\right) \leqslant c e^{n L} d(u, v) \text {, para todo } u, v \in B, n \in \mathbb{N} .
$$

Teorema 2.5.1. Assuma que $\left\{T^{n}: n \in \mathbb{N}\right\}$ é um semigrupo discreto gradient-like. Seja $\mathcal{A}$ seu atrator global e $\left\{y_{1}^{*}, \cdots y_{\mathfrak{p}}^{*}\right\}$ suas soluções estacionárias. Sabemos que

$$
\mathcal{A}=\bigcup_{j=1}^{\mathfrak{p}} W^{\mathrm{u}}\left(y_{j}^{*}\right)
$$

Assuma que existam constantes positivas $C, \varrho$ e $\delta_{0}$ tais que, para $W_{\mathrm{loc}}^{u}\left(y_{j}^{*}\right):=W^{u}\left(y^{*}\right) \cap$ $B_{\delta_{0}}\left(y_{j}^{*}\right)$

$$
\operatorname{dist}_{H}\left(T^{n} u_{0}, W_{\text {loc }}^{u}\left(y_{j}^{*}\right)\right) \leqslant C e^{-n \varrho}
$$


para cada $u_{0} \in B_{\delta_{0}}\left(y_{j}^{*}\right)$ e $n \in \mathbb{N}$ tal que $\gamma_{[0, n]}\left(u_{0}\right) \subset B_{\delta_{0}}\left(y_{j}^{*}\right), 1 \leqslant j \leqslant \mathfrak{p}$. Então, para todo $B \subset X$ limitado existe um $c(B)>0$ tal que

$$
\operatorname{dist}_{H}\left(T^{n} u_{0}, \mathcal{A}\right) \leqslant c(B) e^{-n \varrho}, \text { para todo } u_{0} \in B
$$

Demonstração: A demonstração é fortemente baseada nas propriedades fundamentais de semigrupos gradient-like dados nos Lemas 2.4.5 e 2.4.6.

Do Lema 2.4.6, para $\delta$ suficientemente pequeno, existe $\delta^{\prime}=\delta^{\prime}(\delta)<\delta$ tal que, se $u_{0} \in B_{\delta^{\prime}}\left(y_{i}^{*}\right)$ e para algum $n_{1}>0$

$$
T^{n_{1}} u_{0} \notin B_{\delta}\left(y_{i}^{*}\right)
$$

então

$$
T^{n} u_{0} \notin B_{\delta^{\prime}}\left(y_{i}^{*}\right) \text {, para todo } n \geqslant n_{1} .
$$

Agora, seja $B$ um subconjunto limitado de $X$ e $B_{0}=\gamma^{+}(B)$. Por 2.5.1) e pelo fato do semigrupo possuir um atrator, $B_{0}$ é limitado. Assim, segue do Lema 2.4 .5 que existe $N=N\left(\delta^{\prime}, B_{0}\right)$ tal que, para todo $u_{0} \in B_{0}$

$$
T^{n} u_{0} \in \mathcal{O}_{\delta^{\prime}}=\bigcup_{i=1}^{\mathfrak{p}} B_{\delta^{\prime}}\left(y_{i}^{*}\right) \text { para algum } n \leqslant N .
$$

Logo, dado $u_{0} \in B_{0}$, existem sequências $\left\{n_{-}^{i}\right\}_{i=1}^{M}$ e $\left\{n_{+}^{i}\right\}_{i=1}^{M}, M \leqslant \mathfrak{p}$ e $\left\{y_{i}^{*}\right\}_{i=1}^{M}$ tais que

$$
n_{-}^{1} \leqslant N, \quad n_{-}^{i}-n_{+}^{i-1} \leqslant N, \quad 2 \leqslant i \leqslant M \quad n_{+}^{M}=+\infty
$$

para os quais $T^{n} u_{0} \in \mathcal{O}_{\delta}\left(y_{i}^{*}\right)$, para todo $n \in\left[n_{-}^{i}, n_{+}^{i}\right]$ e $i \in\{1, \ldots, M\}$. Então, por (2.5.3)

$$
\operatorname{dist}_{H}\left(T^{n} u_{0}, \mathcal{A}\right) \leqslant \operatorname{dist}_{H}\left(T^{n} u_{0}, W_{\text {loc }}^{u}\left(y_{i}^{*}\right)\right) \leqslant c_{0}\left(B_{0}\right) e^{-n \varrho} \text {, para todo } n \in\left[n_{-}^{i}, n_{+}^{i}\right],
$$

uma vez que $W_{\text {loc }}^{u}\left(y_{j}^{*}\right) \subset \mathcal{A}$, para todo $j \in 1, \ldots, \mathfrak{p}$. Por outro lado, para $n \in\left[n_{+}^{i-1}, n_{-}^{i}\right]$, 
$n=k+n_{+}^{i-1}$, para algum $k \leqslant N$, e usando 2.5.1 temos que

$$
\begin{aligned}
\operatorname{dist}_{H}\left(T^{n} u_{0}, \mathcal{A}\right) & =\operatorname{dist}_{H}\left(T^{k+n_{+}^{i-1}} u_{0}, \mathcal{A}\right) \\
& =\operatorname{dist}_{H}\left(T^{k} T^{n_{+}^{i-1}} u_{0}, T^{k} \mathcal{A}\right) \\
& \leqslant c_{1}\left(B_{0}\right) e^{k L} \operatorname{dist}_{H}\left(T^{n_{+}^{i-1}} u_{0}, \mathcal{A}\right) \\
& \leqslant c_{1}\left(B_{0}\right) e^{k L} c_{0}\left(B_{0}\right) e^{-n_{+}^{i-1} \varrho}=c\left(B_{0}\right) e^{-n \varrho} .
\end{aligned}
$$

O seguinte resultado sobre atratores exponenciais uniformes para uma família de semigrupos discretos coletivamente assintoticamente compactos vale. Sua demonstração é idêntica à do Teorema 2.5.1 usando os Lemas 2.4.17 e 2.4.18 ao invés dos Lemas 2.4.5 e 2.4 .6 .

Teorema 2.5.2. Seja $X$ um espaço de Banach e $\left\{T_{\eta}^{n}: n \in \mathbb{N}\right\}, \eta \in[0,1]$, uma família de semigrupos discretos coletivamente assintoticamente compactos em X que satisfazem

a) para cada $\eta \in[0,1]\left\{T_{\eta}^{n}: n \in \mathbb{N}\right\}$ tem um atrator global $\mathcal{A}_{\eta}$ e $\overline{\cup_{\eta \in[0,1]} \mathcal{A}_{\eta}}$ é compacto.

b) $\left\{T_{\eta}^{n}: n \in \mathbb{N}\right\}$ tem um número finito de soluçôes estacionárias $\mathcal{E}_{\eta}=\left\{y_{1}^{*, \eta}, \cdots, y_{\mathfrak{p}}^{*, \eta}\right\}$, para todo $\eta \in[0,1]$, e $\sup _{1 \leqslant i \leqslant p} d\left(y_{i}^{*, \eta}, y_{i}^{*, 0}\right) \stackrel{\eta \rightarrow 0}{\longrightarrow} 0$.

c) existem constantes positivas $M$ e $\varrho$ e, para cada $1 \leqslant i \leqslant \mathfrak{p}$, uma vizinhança $B_{\delta_{0}}\left(y_{i}^{*}\right)$ de $y_{i}^{*, 0}$ tal que, para cada $u_{0} \in B_{\delta_{0}}\left(y_{i}^{*}\right)$, enquanto $\gamma_{[0, n]}\left(u_{0}\right) \subset B_{\delta_{0}}\left(y_{i}^{*}\right)$

$$
\operatorname{dist}_{H}\left(T^{n} u_{0}, W_{\eta}^{u}\left(y_{i}^{*, \eta}\right) \cap B_{\delta_{0}}\left(y_{i}^{*}\right)\right) \leqslant M e^{-n \varrho},
$$

d) $d\left(T_{\eta}^{n} u, T_{0}^{n} u\right) \stackrel{\eta \rightarrow 0}{\longrightarrow} 0$ uniformemente para $u$ em subconjuntos compactos de $X$, para cada $n \in \mathbb{N}$.

e) $\left\{T_{0}^{n}: n \in \mathbb{N}\right\}$ é um semigrupo discreto gradient-like.

Então, existe um $\eta_{0}>0$ tal que $\left\{T_{\eta}^{n}: n \in \mathbb{N}\right\}$ é gradient-like, e para cada conjunto limitado $B \subset X$, existe uma constante $c(B)>0$ tal que

$$
\operatorname{dist}_{H}\left(T_{\eta}^{n} u_{0}, \mathcal{A}\right) \leqslant c(B) e^{-n \varrho}, \text { para todo } u_{0} \in B
$$


Encerramos esta seção com um resultado sobre atratores exponenciais para semigrupos discretos gradient-like. A principal diferença aqui é que a atração exponencial de conjuntos instáveis locais não é conhecida para uma situação geral e deve ser assumida.

Teorema 2.5.3. Assuma que $\left\{T^{n}: n \in \mathbb{N}\right\}$ é um semigrupo discreto gradient-like generalizado (veja Definição 2.4.23). Então

$$
\mathcal{A}=\cup_{i=1}^{\mathfrak{p}} W^{u}\left(\Xi_{i}^{*}\right)
$$

Se além disso assumirmos (2.5.1) e que existe $\varrho>0$ e, para cada $1 \leqslant i \leqslant \mathfrak{p}$, uma vizinhança $V_{i}$ de $\Xi_{i}^{*}$ tal que, para qualquer $u_{0} \in V_{i}$, enquanto $T^{n} u_{0} \in V_{i}$

$$
\operatorname{dist}_{H}\left(T^{n} u_{0}, W^{u}\left(\Xi^{*}\right)\right) \leqslant M e^{-n \varrho},
$$

para conjuntos limitados $B \subset X$, existe uma constante $c(B)>0$ tal que

$$
\operatorname{dist}_{H}\left(T^{n} u_{0}, \mathcal{A}\right) \leqslant c(B) e^{-n \varrho}, \text { para todo } u_{0} \in B
$$

A demonstração deste resultado é idêntica à prova do Teorema 2.5.1 usando as seguintes generalizações dos Lemas 2.4.5 e 2.4.6 com demonstrações idênticas.

Lema 2.5.4. Seja $\left\{T^{n}: n \in \mathbb{N}\right\}$ um semigrupo discreto gradient-like generalizado com um número finito de conjuntos invariantes isolados $\mathcal{E}=\left\{\Xi_{1}^{*}, \cdots \Xi_{\mathfrak{p}}^{*}\right\}$ e um atrator global A. Dado $\delta<\delta_{0}$ e $B \subset X$ limitado, existe um $N=N(\delta, B)>0$ tal que $\left\{T^{n} u_{0}: 0 \leqslant n \leqslant\right.$ $N\} \cap \cup_{i=1}^{\mathfrak{p}} \mathcal{O}_{\delta}\left(\Xi_{i}^{*}\right) \neq \emptyset$ para todo $u_{0} \in B$.

Lema 2.5.5. Seja $\left\{T^{n}: n \in \mathbb{N}\right\}$ um semigrupo com atrator global $\mathcal{A}$ e um número finito de conjuntos invariantes isolados $\mathcal{E}=\left\{\Xi_{1}^{*}, \cdots \Xi_{\mathfrak{p}}^{*}\right\}$ em $\mathcal{A}$. Dado $0<\delta<\delta_{0}$, existe um $\delta^{\prime}>0$ tal que, se para algum $1 \leqslant i \leqslant n$, dist $\left(u_{0}, \Xi_{i}^{*}\right)<\delta^{\prime}$ e, para algum $n_{1}>0$, $\operatorname{dist}\left(T^{n_{1}} u_{0}, \Xi_{i}^{*}\right) \geqslant \delta$, então $\operatorname{dist}\left(T^{n} u_{0}, \Xi_{i}^{*}\right)>\delta^{\prime}$ para todo $n \geqslant n_{1}$. 


\subsection{Taxa de convergência de atratores exponenciais}

Nosso próximo resultado diz que a atração exponencial local uniforme garante que a taxa de convergência de semigrupos não-lineares determinam a taxa de convergência dos atratores. A garantia da taxa de convergência exponencial para atratores é muito importante quando tratamos de problemas de aplicação, uma vez que é de grande valia sabermos como os atratores de um semigrupo se comportam à medida que o tempo transcorre.

Teorema 2.6.1. Assuma que $\left\{T_{\eta}^{n}: n \in \mathbb{N}\right\}$ é uma familia de semigrupos discretos tendo atratores globais $\mathcal{A}_{\eta}, \eta \in[0,1]$. Assuma que existam $\eta_{0}>0, \varrho>0$, um conjunto limitado $B_{0} \supset \cup_{\eta \in\left[0, \eta_{0}\right]} \mathcal{A}_{\eta}$ e uma constante $c=c\left(B_{0}\right)>0$ tais que

$$
\operatorname{dist}_{H}\left(T_{\eta}^{n} B_{0}, \mathcal{A}_{\eta}\right) \leqslant c e^{-n \varrho}, \eta \in\left[0, \eta_{0}\right]
$$

Assuma também que existam c e $L>0$ tais que

$$
d\left(T_{\eta}^{n} u, T_{0}^{n} v\right) \leqslant c e^{n L}(d(u, v)+\eta), \text { para todo } u, v \in B_{0}, \forall \eta \in\left[0, \eta_{0}\right]
$$

Então,

$$
\operatorname{dist}_{H}\left(\mathcal{A}_{\eta}, \mathcal{A}_{0}\right)+\operatorname{dist}_{H}\left(\mathcal{A}_{0}, \mathcal{A}_{\eta}\right) \leqslant \bar{c} \eta^{\frac{\varrho}{\varrho+L}}
$$

Demonstração: Primeiro notemos que dado $\epsilon>0$, se $\frac{1}{\varrho} \log \frac{1}{\epsilon}+\frac{1}{\varrho} \log c \in[n, n+1)$ temos que

$$
\operatorname{dist}_{H}\left(T_{\eta}^{n} B_{0}, \mathcal{A}_{\eta}\right)<e^{\varrho} \epsilon, \forall \eta \in\left[0, \eta_{0}\right]
$$

pois temos $\left(\frac{c}{\epsilon}\right)^{\frac{1}{\rho}}<e^{n+1}$, e assim $c e^{-n \rho}<e^{\rho} \epsilon$. Por 2.6.2

$$
\begin{aligned}
& \operatorname{dist}_{H}\left(T_{\eta}^{n} B_{0}, T_{0}^{n} B_{0}\right)=\sup _{x \in B_{0}} \inf _{y \in B_{0}} d\left(T_{\eta}^{n} x, T_{0}^{n} y\right) \leqslant \sup _{x \in B_{0}} d\left(T_{\eta}^{n} x, T_{0}^{n} x\right) \leqslant c \eta e^{n L} \\
& \operatorname{dist}_{H}\left(T_{0}^{n} B_{0}, T_{\eta}^{n} B_{0}\right)=\sup _{x \in B_{0}} \inf _{y \in B_{0}} d\left(T_{0}^{n} x, T_{\eta}^{n} y\right) \leqslant \sup _{x \in B_{0}} d\left(T_{0}^{n} x, T_{\eta}^{n} x\right) \leqslant c \eta e^{n L} .
\end{aligned}
$$

Note que, como $\mathcal{A}_{\eta} \subset B_{0}$ e $\mathcal{A}_{0} \subset B_{0}$ temos que $\mathcal{A}_{\eta}=T_{\eta}^{n} \mathcal{A}_{\eta} \subset T_{\eta}^{n} B_{0}$ e $\mathcal{A}_{0}=T_{0}^{n} \mathcal{A}_{0} \subset T_{0}^{n} B_{0}$. 
Se $n \in \mathbb{N}$ é tal que $\frac{1}{\varrho} \log \frac{1}{\epsilon}+\frac{1}{\varrho} \log c \in[n, n+1)$, segue de 2.6.2 e 2.6.3 que

$$
\begin{aligned}
\operatorname{dist}_{H}\left(\mathcal{A}_{\eta}, \mathcal{A}_{0}\right) & \leqslant \operatorname{dist}_{H}\left(\mathcal{A}_{\eta}, T_{\eta}^{n} B_{0}\right)+\operatorname{dist}_{H}\left(T_{\eta}^{n} B_{0}, T_{0}^{n} B_{0}\right)+\operatorname{dist}_{H}\left(T_{0}^{n} B_{0}, \mathcal{A}_{0}\right) \\
& \leqslant \operatorname{dist}_{H}\left(T_{\eta}^{n} B_{0}, T_{0}^{n} B_{0}\right)+e^{\varrho} \epsilon \leqslant c e^{n L} \eta+e^{\rho} \epsilon \leqslant c^{1+\frac{L}{\varrho}} \epsilon^{-\frac{L}{\varrho}} \eta+e^{\varrho} \epsilon
\end{aligned}
$$

$\mathrm{e}$

$$
\begin{aligned}
\operatorname{dist}_{H}\left(\mathcal{A}_{0}, \mathcal{A}_{\eta}\right) & \leqslant \operatorname{dist}_{H}\left(\mathcal{A}_{0}, T_{0}^{n} B_{0}\right)+\operatorname{dist}_{H}\left(T_{0}^{n} B_{0}, T_{\eta}^{n} B_{0}\right)+\operatorname{dist}_{H}\left(T_{\eta}^{n} B_{0}, \mathcal{A}_{\eta}\right) \\
& \leqslant \operatorname{dist}_{H}\left(T_{0}^{n} B_{0}, T_{\eta}^{n} B_{0}\right)+e^{\rho} \epsilon \leqslant c e^{n L} \eta+e^{\varrho} \epsilon \leqslant c^{1+\frac{L}{\varrho}} \epsilon^{-\frac{L}{\varrho}} \eta+e^{\varrho} \epsilon .
\end{aligned}
$$

Consequentemente, se $n \in \mathbb{N}$ é tal que $\frac{1}{\varrho} \log \frac{1}{\epsilon}+\frac{1}{\varrho} \log c \in[n, n+1)$,

$$
\operatorname{dist}_{H}\left(\mathcal{A}_{\eta}, \mathcal{A}_{0}\right)+\operatorname{dist}_{H}\left(\mathcal{A}_{0}, \mathcal{A}_{\eta}\right) \leqslant 2 c^{1+\frac{L}{\varrho}} \epsilon^{-\frac{L}{\varrho}} \eta+2 e^{\varrho} \epsilon
$$

Minimizando o lado direito da expressão acima para $\epsilon \in(0, \infty)$ encontramos que $\epsilon=$ $c\left(\frac{L}{\varrho \varrho^{\varrho}}\right)^{\frac{\varrho}{\varrho+L}} \eta^{\frac{\varrho}{\varrho+L}}$ é ponto de mínimo. Como o lado esquerdo da expressão acima é independente de $\epsilon$, temos que

$$
\operatorname{dist}_{H}\left(\mathcal{A}_{\eta}, \mathcal{A}_{0}\right)+\operatorname{dist}_{H}\left(\mathcal{A}_{0}, \mathcal{A}_{\eta}\right) \leqslant 2 c\left(\left(\frac{L}{\varrho e^{\varrho}}\right)^{\frac{-L}{\varrho+L}}+\left(\frac{L e^{L}}{\rho}\right)^{\frac{\varrho}{\varrho+L}}\right) \eta^{\frac{\varrho}{\varrho+L}}
$$

e a prova está completa. 
Capítulo

3

\section{Dimensão fractal e dimensão de} Hausdorff de atratores

Muito do apresentado aqui está contido no trabalho de Mañé [17]. Somente algumas observações sobre semigrupos gradient-like não estão neste trabalho. Este capítulo tenta fazer com que estes resultados se tornem acessíveis para iniciantes, detalhando muitos passos pulados em Mañé [17].

Os atratores para semigrupos discretos em espaços de Banach de dimensão infinita têm dimensão de Hausdorff finita. Nesta seção apresentamos estes resultados. Antes de continuarmos, vamos brevemente relembrar as noções de dimensão de Hausdorff e dimensão fractal.

Uma das motivações para o estudo da dimensão fractal de atratores é o seguinte: mostraremos que conjuntos com dimensão fractal finita podem ser projetados num espaço de dimensão finita de maneira injetiva, e mostraremos também que os atratores para semigrupos discretos têm dimensão fractal finita. Logo, dado um problema discreto num espaço de Banach $X$ de dimensão infinita

$$
x_{n+1}=T x_{n},
$$

cujo semigrupo associado $\left\{T^{n}: n \in \mathbb{N}\right\}$ tem atrator global $\mathcal{A}$, podemos levá-lo a um espaço de dimensão finita $Y$, por meio de uma projeção $P: X \rightarrow Y$ injetiva em $\mathcal{A}$ e 
considerar o problema

$$
P x_{n+1}=P T x_{n}
$$

Se denotarmos $y_{n}=P x_{n}$, podemos reescrever o problema na forma

$$
y_{n+1}=P T P^{-1} y_{n}
$$

desde que a inversa de $P$ possa ser definida em todo o conjunto $Y$ de maneira contínua, estendendo a inversa desta mesma projeção sobre a imagem do atrator $P(\mathcal{A})$. Uma vez feito este processo, conseguimos um homeomorfismo entre o atrator do problema de dimensão infinita e o atrator do problema de dimensão finita, sendo assim suficiente realizar o estudo dos atratores para sistemas em espaços de dimensão finita.

O grande problema aqui, que ainda continua sem solução, é encontrar uma extensão contínua da inversa da projeção $P: \mathcal{A} \rightarrow P(\mathcal{A})$ em todo $Y$. Uma vez construída esta inversa, toda a teoria de atratores pode ser reduzida ao caso finito dimensional.

\subsection{Dimensão, dimensão de Hausdorff e dimensão fractal}

Nesta seção apresentamos os conceitos básicos de dimensão que serão usados no que segue, seguindo os livros de Falconer [10] e Folland [11].

Seja $K$ um espaço topológico. Dizemos que $K$ tem dimensão finita se existe um inteiro $n$ tal que, para toda a cobertura aberta $\mathcal{U}$ de $K$, existe uma cobertura aberta $\mathcal{U}^{\prime}$ de $K$ refinando $\mathcal{U}$ com a propriedade de que cada ponto de $K$ pertence a no máximo $n+1$ subconjuntos de $\mathcal{U}^{\prime}$. Neste caso, a dimensão topológica $\operatorname{dim}(K)$ de $K$ é o mínimo $n$ com esta propriedade. Este conceito tem a propriedade de que a dimensão de $\mathbb{R}^{n}$ é $n$ e, se $K$ é um espaço compacto de dimensão finita, então é homeomorfo a algum subconjunto de $\mathbb{R}^{n} \operatorname{com} n=2 \operatorname{dim}(K)+1$.

A seguir apresentamos uma revisão sobre medida de Hausdorff e dimensão de Hausdorff de um espaço métrico $(X, \rho)$, para uma abordagem mais detalhada vide Falconer[10] e Folland [11]. Uma medida exterior $\mu^{*}: 2^{X} \rightarrow[0, \infty]$ é uma função que satisfaz
i) $\mu^{*}(\emptyset)=0$;
ii) $\mu^{*}(A) \leqslant \mu^{*}(B)$, sempre que $A \subset B$;
iii) $\mu^{*}\left(\cup_{j=1}^{\infty} A_{j}\right) \leqslant \sum_{j=1}^{\infty} \mu^{*}\left(A_{j}\right)$ para qualquer sequência $\left\{A_{j}\right\}_{j=1}^{\infty}$ em $2^{X}$. 
Um conjunto $A \subset X$ é dito $\mu^{*}$-mensurável se para cada $E \subset X$

$$
\mu^{*}(E)=\mu^{*}(E \cap A)+\mu^{*}\left(E \cap A^{c}\right)
$$

A seguir apresentamos a definição e algumas propriedades básicas da dimensão de Hausdorff. Para um espaço métrico $(X, \rho), \alpha \geqslant 0$, e $\epsilon>0$. Se $A \subset X$, seja

$$
\mu_{\epsilon}^{(\alpha)}(A)=\inf \left\{\sum_{i=1}^{\infty}\left(\operatorname{diam}\left(B_{i}\right)\right)^{\alpha}: A \subset \cup_{i=1}^{\infty} B_{i}, \operatorname{diam}\left(B_{i}\right)<\epsilon\right\}
$$

com a convenção $\inf \emptyset=\infty$. Como $\mu_{\epsilon}^{(\alpha)}(A)$ cresce quando $\epsilon$ decresce definimos

$$
\mu^{(\alpha)}(A)=\lim _{\epsilon \rightarrow 0} \mu_{\epsilon}^{(\alpha)}(A)
$$

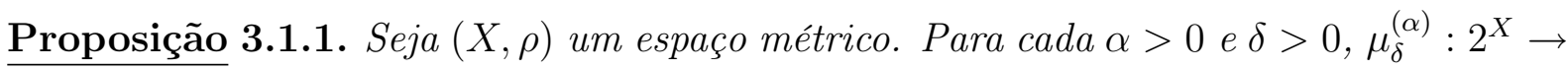
$[0, \infty]$ é uma medida exterior.

Demonstração: Fixe $\delta>0$. Claramente $\mu_{\delta}^{(\alpha)}(\emptyset)=0$ e $\mu_{\delta}^{(\alpha)}(A) \leqslant \mu_{\delta}^{(\alpha)}(B)$ sempre que $A \subset B$. Se $\epsilon>0,\left\{A_{j}\right\}_{j=1}^{\infty}$ é uma sequência em $2^{X}$ e para cada $j \in \mathbb{N}^{*}$ existe uma sequência $\left\{B_{i}^{j}\right\}_{i=1}^{\infty} \operatorname{com} A_{j} \subset \bigcup_{i=1}^{\infty} B_{i}^{j}, \operatorname{diam}\left(B_{i}^{j}\right)<\delta$, para todo $i \in \mathbb{N}^{*}$, e $\sum_{i=1}^{\infty}\left(\operatorname{diam}\left(B_{i}^{j}\right)\right)^{\alpha} \leqslant$ $\mu_{\delta}^{\alpha}\left(A_{j}\right)+\epsilon 2^{-j}$, então $\bigcup_{j=1}^{\infty} A_{j} \subset \bigcup_{j, i=1}^{\infty} B_{i}^{j} \mathrm{e}$

$$
\mu_{\delta}^{(\alpha)}\left(\bigcup_{j=1}^{\infty} A_{j}\right) \leqslant \sum_{i, j=1}^{\infty}\left(\operatorname{diam}\left(B_{i}^{j}\right)\right)^{\alpha} \leqslant \sum_{j=1}^{\infty} \mu_{\delta}^{(\alpha)}\left(A_{j}\right)+\epsilon .
$$

Segue que $\mu_{\delta}^{(\alpha)}\left(\bigcup_{j=1}^{\infty} A_{j}\right) \leqslant \sum_{j=1}^{\infty} \mu_{\delta}^{(\alpha)}\left(A_{j}\right)$. O resultado segue agora imediatamente.

Com isto temos que

Teorema 3.1.2. Seja $(X, \rho)$ um espaço métrico. Para cada $\alpha>0, \mu^{(\alpha)}: 2^{X} \rightarrow[0, \infty]$ é uma medida exterior.

Demonstração: Segue imediatamente da Proposição 3.1 .1 que $\mu^{(\alpha)}$ é uma medida exterior.

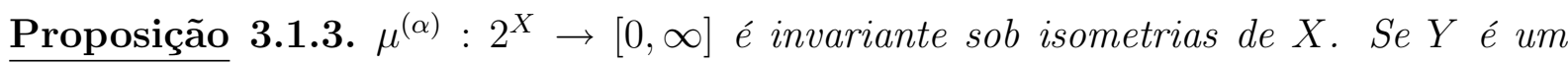


conjunto qualquer e $f, g: Y \rightarrow X$ são tais que

$$
\rho(f(y), f(z)) \leqslant C \rho(g(y), g(z)), \forall y, z \in Y,
$$

então $\mu^{(\alpha)}(f(A)) \leqslant C^{\alpha} \mu^{(\alpha)}(g(A))$ para todo $A \subset Y$.

Demonstração: A primeira afirmação é óbvia da definição de $\mu^{(\alpha)}$. Se $\mu^{(\alpha)}(g(A))<\infty$, dado $\epsilon>0$, existe $\delta_{\epsilon}>0$ tal que, para todo $0<\delta<\delta_{\epsilon}$, existe uma cobertura de $g(A)$ por conjuntos $B_{j}$ tal que $\operatorname{diam}\left(B_{j}\right) \leqslant C^{-1} \delta$ e

$$
\sum_{j=1}^{\infty}\left(\operatorname{diam}\left(B_{j}\right)\right)^{\alpha} \leqslant \mu^{(\alpha)}(g(A))+\epsilon
$$

Os conjuntos $B_{j^{\prime}}=f\left(g^{-1}\left(B_{j}\right)\right)$ cobrem $f(A)$, e se $g^{-1}\left(B_{j}\right)=F_{j}$, então $g\left(F_{j}\right) \subset B_{j}$ e $f\left(F_{j}\right)=B_{j^{\prime}}$ e $\operatorname{diam}\left(B_{j^{\prime}}\right) \leqslant C \operatorname{diam}\left(B_{j}\right) \leqslant \delta, \mathrm{e}$

$$
\mu_{\delta}^{(\alpha)}(f(A)) \leqslant C^{\alpha} \mu^{(\alpha)}(g(A))+C^{\alpha} \epsilon
$$

O resultado agora segue fazendo $\delta \rightarrow 0$ e fazendo $\epsilon \rightarrow 0$.

Corolário 3.1.4. Seja $f: X \rightarrow X$ uma função Lipschitz contínua com constante de Lipschitz $C \geqslant 0$ e $A \subset X$. Então $\mu^{(\alpha)}(f(A)) \leqslant C^{\alpha} \mu^{(\alpha)}(A)$.

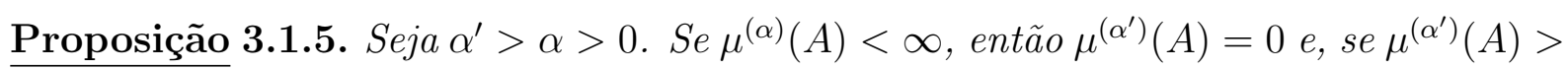
0 , então $\mu^{(\alpha)}(A)=\infty$.

Demonstração: É suficiente provar a primeira afirmação, uma vez que a segunda é sua contrapositiva. Se $\mu^{(\alpha)}(A)<\infty$, para qualquer $\delta>0$ existe $\left\{B_{j}\right\}_{j \in \mathbb{N}} \operatorname{com} A \subset \cup_{j=1}^{\infty} B_{j}$, $\operatorname{diam}\left(B_{j}\right) \leqslant \delta, \mathrm{e}$

$$
\sum_{j=1}^{\infty}\left(\operatorname{diam}\left(B_{j}\right)\right)^{\alpha} \leqslant \mu^{(\alpha)}(A)+1
$$

Mas para $\alpha^{\prime}>\alpha$,

$$
\sum_{j=1}^{\infty}\left(\operatorname{diam}\left(B_{j}\right)\right)^{\alpha^{\prime}} \leqslant \delta^{\alpha^{\prime}-\alpha} \sum_{j=1}^{\infty}\left(\operatorname{diam}\left(B_{j}\right)\right)^{\alpha} \leqslant \delta^{\alpha^{\prime}-\alpha}\left[\mu^{(\alpha)}(A)+1\right]
$$

$\log 0 \mu_{\delta}^{\left(\alpha^{\prime}\right)} \leqslant \delta^{\alpha^{\prime}-\alpha}\left[\mu^{(\alpha)}(A)+1\right] \stackrel{\delta \rightarrow 0}{\longrightarrow} 0$ e $\mu^{\left(\alpha^{\prime}\right)}(A)=0$. 
Definição 3.1.6. Para qualquer $A \subset X$, a dimensão de Hausdorff de $A$ é definida pelo número real não-negativo dado por

$$
\inf \left\{\alpha \geqslant 0: \mu^{(\alpha)}(A)=0\right\}=\sup \left\{\alpha \geqslant 0: \mu^{(\alpha)}(A)=\infty\right\}
$$

Observação 3.1.7. Sabemos que (vide Kahane [14]) $\operatorname{dim}(K) \leqslant \operatorname{dim}_{H}(K)$.

Proposição 3.1.8. Seja $f: X \rightarrow X$ uma função Lipschitz contínua e $A \subset X$. Então $\operatorname{dim}_{H}(f(A)) \leqslant \operatorname{dim}_{H}(A)$.

Demonstração: Pelo Corolário 3.1.4, temos que se $\mu^{\alpha}(A)=0$ então $\mu^{\alpha}(f(A))=0$. $\operatorname{Assim}_{\operatorname{dim}_{H}}(f(A)) \leqslant \operatorname{dim}_{H}(A)$.

Corolário 3.1.9. Seja $f: X \rightarrow X$ uma função Lipschitz contínua, $A \subset X$ e $G(f, A)=$ $\{(x, f(x)): x \in A\}$ o gráfico de $f$ restrito à $A$. Então $\operatorname{dim}_{H}(G(f, A))=\operatorname{dim}_{H}(A)$.

Demonstração: Sabemos que as aplicações $A \ni x \mapsto(x, f(x)) \in G(f, A)$ e $G(f, A) \ni$ $(x, f(x)) \mapsto x \in A$ são Lipschitz contínuas, e da proposição acima segue que

$$
\operatorname{dim}_{H}(G(f, A)) \leqslant \operatorname{dim}_{H}(A) \leqslant \operatorname{dim}_{H}(G(f, A)) .
$$

Proposição 3.1.10. Seja $\left\{A_{j}\right\}_{j \in \mathbb{N}}$ uma sequência de conjuntos em $X$ e seja $A=\bigcup_{j \in \mathbb{N}} A_{j}$. Então

$$
\operatorname{dim}_{H}\left(\bigcup_{j \in \mathbb{N}} A_{j}\right) \leqslant \sup _{j \in \mathbb{N}} \operatorname{dim}_{H}\left(A_{j}\right)
$$

Demonstração: Seja $\alpha>\sup _{j \in \mathbb{N}} \operatorname{dim}_{H}\left(A_{j}\right)$, assim

$$
\mu^{\text {alpha }}(A) \leqslant \sum_{j \in \mathbb{N}} \mu^{\alpha}\left(A_{j}\right)=0
$$

Portanto $\operatorname{dim}_{H}(A) \leqslant \alpha$. Como $\alpha>\sup _{j \in \mathbb{N}} \operatorname{dim}_{H}\left(A_{j}\right)$ é arbitrário, temos o resultado.

Vamos agora nos voltar para os atratores de tipo gradiente em espaços de Banach. Seja $\mathcal{E}=\left\{e_{1}^{*}, \ldots, e_{p}^{*}\right\}$ o conjunto de equilíbrio do semigrupo $\left\{T^{n}: n \in \mathbb{N}\right\}$, e suponha que 
$T \in \mathcal{C}(X)$ é uma aplicação Lipschitz contínua. Sabemos que o atrator global $\mathcal{A}$ é dado por

$$
\mathcal{A}=\bigcup_{j=1}^{p} W^{u}\left(e_{j}^{*}\right)
$$

Suponha que o conjunto instável $W_{\text {loc }}^{u}\left(e_{i}^{*}\right)$ de cada ponto de equilíbrio é o gráfico de uma função Lipschitz contínua com domínio $Q X$, onde $Q$ é uma projeção de posto finito. Do Corolário 3.1.9 e da Proposição 3.1.8, sabemos que

$$
\begin{aligned}
& \operatorname{dim}_{H}\left(W_{\text {loc }}^{u}\left(e_{i}^{*}\right)\right)=\operatorname{dim}_{H}(Q X)<\infty, \text { para cada } i=1, \ldots, p, \\
& \operatorname{dim}_{H}\left(T^{n} W_{\text {loc }}^{u}\left(e_{i}^{*}\right)\right) \leqslant \operatorname{dim}_{H}\left(W_{\text {loc }}^{u}\left(e_{i}^{*}\right)\right) .
\end{aligned}
$$

Facilmente, vemos que $W^{u}\left(e_{i}^{*}\right)=\bigcup_{n=0}^{\infty} T^{n} W_{\text {loc }}^{u}\left(e_{i}^{*}\right)$ e, utilizando a Proposição 3.1.10. temos

$$
\begin{aligned}
\operatorname{dim}_{H}(Q X) & =\operatorname{dim}_{H}\left(W_{\mathrm{loc}}^{u}\left(e_{i}^{*}\right)\right) \leqslant \operatorname{dim}_{H}\left(W^{u}\left(e_{i}^{*}\right)\right)= \\
& =\operatorname{dim}_{H}\left(\bigcup_{n=0}^{\infty} T^{n} W_{\mathrm{loc}}^{u}\left(e_{i}^{*}\right)\right) \leqslant \sup _{n \in \mathbb{N}} \operatorname{dim}_{H}\left(T^{n} W_{\mathrm{loc}}^{u}\left(e_{i}^{*}\right)\right) \leqslant \\
& \leqslant \operatorname{dim}_{H}\left(W_{\mathrm{loc}}^{u}\left(e_{i}^{*}\right)\right)=\operatorname{dim}_{H}(Q X),
\end{aligned}
$$

e portanto $\operatorname{dim}_{H}\left(W^{u}\left(e_{i}^{*}\right)\right)=\operatorname{dim}_{H}(Q X)$, para todo $i=1, \ldots, p$.

Assim, como $\mathcal{A}=\bigcup_{i=1}^{p} W^{u}\left(e_{i}^{*}\right)$, temos que

$$
\operatorname{dim}_{H}(\mathcal{A})=\operatorname{dim}_{H}(Q X)
$$

Portanto, atratores do tipo gradiente em espaços de Banach têm dimensão de Hausdorff finita. Infelizmente, isso não nos dá muita informação sobre o atrator, no sentido de tratálo de fato como um objeto de dimensão (algébrica) finita. Para isto, vamos introduzir o conceito de dimensão fractal, que nos permitirá inserir o atrator de maneira injetiva em um espaço vetorial de dimensão finita.

Seja $K$ um espaço métrico compacto. Defina $N(r, K)$ como o número mínimo de bolas de raio $r$ necessário para cobrir $K$. A dimensão fractal $c(K)$ de $K$ é definida por:

$$
c(K)=\limsup _{r \rightarrow 0} \frac{\log N(r, K)}{\log (1 / r)} .
$$

Alternativamente, $c(K)$ é o menor número real tal que, para todo $\epsilon>0$ existe $\delta>0$ tal 
que

$$
N(r, K) \leqslant\left(\frac{1}{r}\right)^{c(K)+\epsilon}, 0<r<\delta .
$$

Como podemos ver, multiplicando a expressão acima por $r^{\alpha}$ obtemos

$$
\mu_{r}^{(\alpha)}(K) \leqslant N(r, K) r^{\alpha} \leqslant\left(\frac{1}{r}\right)^{c(K)+\epsilon-\alpha} \quad, \quad 0<r<\delta .
$$

Assim, $\mu_{r}^{(c(K)+\eta)}(K) \leqslant\left(\frac{1}{r}\right)^{\epsilon-\eta}$ para $0<r<\delta$ e para todo $\eta>0$. Fazendo $r \rightarrow 0$ temos $\mu^{(c(K)+\eta)}(K)=0$, para todo $0<\eta<\epsilon$. Da definição de dimensão de Hausdorff, segue facilmente que

$$
\operatorname{dim}_{H}(K) \leqslant c(K)
$$

Mais ainda, $c(K)$ e $\operatorname{dim}_{H}(K)$ podem ser diferentes (vide Mañé [17]).

Lema 3.1.11. Seja $X$ um espaço vetorial normado e $K_{1}, K_{2}$ subconjuntos compactos de $X$. Se $c\left(K_{i}\right)<\infty, i=1,2$ então $c\left(K_{1}+K_{2}\right) \leqslant c\left(K_{1}\right)+c\left(K_{2}\right)$.

Demonstração: Note que, se $N_{i}=N\left(r, K_{i}\right)$ existem $x_{1}^{i}, \cdots, x_{N}^{i}$ em $K$ tais que $K_{i} \subset$ $\cup_{j=1}^{N_{i}} B_{r}\left(x_{j}^{i}\right)$ e consequentemente $K_{1}+K_{2} \subset \cup_{i=1}^{N_{2}} \cup_{j=1}^{N_{1}}\left(B_{r}\left(x_{j}^{1}\right)+B_{r}\left(x_{i}^{2}\right)\right)$. Como diam $\left(B_{r}\left(x_{j}^{1}\right)+\right.$ $\left.B_{r}\left(x_{i}^{2}\right)\right) \leqslant 2 r$ temos que $N\left(2 r, K_{1}+K_{2}\right) \leqslant N\left(r, K_{1}\right) N\left(r, K_{2}\right)$ e consequentemente

$$
\begin{aligned}
c\left(K_{1}+K_{2}\right) & =\limsup _{r \rightarrow 0} \frac{\log N\left(2 r, K_{1}+K_{2}\right)}{\log (1 / 2 r)} \leqslant \limsup _{r \rightarrow 0} \frac{\log N\left(r, K_{1}\right) N\left(r, K_{2}\right)}{\log (1 / 2 r)} \\
& \leqslant \limsup _{r \rightarrow 0} \frac{\log N\left(r, K_{1}\right)}{\log (1 / r)}+\limsup _{r \rightarrow 0} \frac{\log N\left(r, K_{2}\right)}{\log (1 / r)}=c\left(K_{1}\right)+c\left(K_{2}\right) .
\end{aligned}
$$

Como consequência imediata do Lema 3.1.11 temos que

Corolário 3.1.12. Seja $X$ um espaço vetorial normado e $K$ um subconjunto compacto de $X$. Se $c(K)<\infty$, então $c(K-K) \leqslant 2 c(K)$.

\subsection{Projeções de conjuntos compactos com dimensão frac-}

\section{tal finita}

Como já mencionamos anteriormente, gostaríamos de enxergar os atratores de semigrupos como objetos de dimensão finita, mesmo quando o semigrupo está num espaço de 
Banach de dimensão infinita. Para isso vamos mostrar que conjuntos compactos que têm dimensão fractal finita podem ser colocados, de maneira injetiva, num espaço vetorial de dimensão finita.

Antes de mostrarmos alguns resultados sobre dimensionalidade finita de conjuntos compactos invariantes para certas aplicações não-lineares vamos provar alguns resultados básicos de análise funcional.

Seja $X$ um espaço de Banach e $Y$ um subespaço de dimensão finita de $X$. Denote por $\mathcal{P}(X, Y)$ o subconjunto de $\mathcal{L}(X)$ consistindo das projeções com imagem $Y$.

Seja $X$ um espaço de Banach, $\left\{K_{n}\right\}_{n \in \mathbb{N}}$ uma sequência de subconjuntos compactos de $X$ e $K=\cup_{n \in \mathbb{N}} K_{n}$.

Lema 3.2.1. Dado $r>0, n \in \mathbb{N}$, se

$$
A_{n, r}=\left\{v-w \in X: v, w \in K_{n} e\|v-w\|_{X} \geqslant r\right\}
$$

então $A_{n, r}$ é um subconjunto compacto de $X$.

Demonstração: Primeiramente mostremos que $A_{n, r}$ é fechado. De fato, $y_{k}=v_{k}-w_{k} \stackrel{k \rightarrow \infty}{\longrightarrow}$ $y$, com $v_{k}, w_{k} \in K_{n},\left\|v_{k}-w_{k}\right\|_{X}$. Usando a compacidade de $K_{n}$, podemos assumir que, passando a subsequências, que $v_{k} \stackrel{k \rightarrow \infty}{\longrightarrow} v \in K_{n}$ e $w_{k} \stackrel{k \rightarrow \infty}{\longrightarrow} w \in K_{n}$. Portanto $y=v-w$ e $\|v-w\|_{X} \geqslant r$ e $y \in A_{n, r}$. O que prova que $A_{n, r}$ é fechado.

Agora notemos que, $A_{n, r} \subset K_{n}-K_{n}$. Como $X \times X \ni(x, y) \mapsto x-y \in X$ é contínua, segue que $K_{n}-K_{n}$ é compacto e consequentemente $A_{n, r}$ é um compacto em $X$.

Lema 3.2.2. Defina

$$
\mathcal{P}_{n, r}=\left\{P \in \mathcal{P}(X, Y): \operatorname{diam}\left(P^{-1}(y) \cap K_{n}\right)<r, \forall y \in Y\right\}
$$

Se $P \in \mathcal{P}(X, Y)$, então $P \in P_{n, r}$ se e somente se $P^{-1}(0) \cap A_{n, r}=\emptyset$

Demonstração: Assuma que existe um $y \in P^{-1}(0) \cap A_{n, r}$, então $P y=0$ e existem $v, w \in$ $K_{n}$ tais que $y=v-w,\|v-w\|_{X} \geqslant r$. Se $z=P v=P w \operatorname{então} \operatorname{diam}\left(P^{-1}(z) \cap K_{n}\right) \geqslant r$ e $P \notin P_{n, r}$.

Por outro lado, se $P^{-1}(0) \cap A_{n, r}=\emptyset$, para todo $y \in Y$ e $v, w \in P^{-1}(y) \cap K_{n}$, temos que $v-w \in P^{-1}(0)(P(v-w)=y-y=0)$ e portanto devemos ter $\|v-w\|_{X}<r$. Como $P^{-1}(y) \cap K_{n}$ é compacto, segue que $\operatorname{diam}\left(P^{-1}(y) \cap K_{n}\right)<r$. Isto completa a demonstração. 
Lema 3.2.3. Se $P_{n, r}$ definida por (3.2.1), então $P_{n, r}$ é aberto em $\mathcal{P}(X, Y)$ com a topologia uniforme de operadores.

Demonstração: A demonstração deste resultado está baseada na caracterização de $P_{n, r}$ dado no Lema 3.2.2. Dada uma projeção $P \in P_{n, r}$, notemos que $\epsilon=\operatorname{dist}\left(P^{-1}(0), A_{n, r}\right)>$ 0 . Escolha $s>0$ tal que $B_{s}(0) \supset A_{n, r}$ e seja $\bar{P} \in \mathcal{P}(X, Y)$ tal que $\|P-\bar{P}\|_{\mathcal{L}(X)}<\frac{\epsilon}{s}$. Então

$$
\begin{aligned}
\inf _{x \in \bar{P}^{-1}(0)} \operatorname{dist}\left(x, A_{n, r}\right)=: \operatorname{dist}\left(\bar{P}^{-1}(0), A_{n, r}\right) & =\operatorname{dist}\left((I-\bar{P}) B_{s}(0), A_{n, r}\right) \\
& \geqslant \operatorname{dist}\left((I-P) B_{s}(0), A_{n, r}\right)-\sup _{x \in B_{s}(0)}\|\bar{P} x-P x\|_{X} \\
& =\operatorname{dist}\left(P^{-1}(0), A_{n, r}\right)-s\|\bar{P}-P\|_{\mathcal{L}(X)}>0 .
\end{aligned}
$$

Segue que $\bar{P}^{-1}(0) \cap A_{n, r}=\emptyset$ e $\bar{P} \in P_{n, r}$, provando que $P_{n, r}$ é aberto.

No que segue, se $X$ é um espaço vetorial, denotaremos por $X^{*}$ o seu dual; isto é, o conjunto dos funcionais lineares contínuos de $X$ em $\mathbb{R}$ (ou $\mathbb{C}$ ).

Lema 3.2.4. Seja $X$ um espaço de Banach e $\left\{A_{n}\right\}_{n \in \mathbb{N}}$ uma sequência de subconjuntos compactos de $X$. Se $A=\cup_{n \in \mathbb{N}} A_{n}$, existe uma sequência $\left\{\phi_{i}\right\}_{i \in \mathbb{N}}$ em $X^{*}$ tal que, se a $\in A$ e $\phi_{i}(a)=0$ para todo $i \in \mathbb{N}$, então $a=0$.

Demonstração: Agora consideremos o fecho $W$ do subespaço de $X$ gerado por $A$. Como $A$ é uma união enumerável de conjuntos compactos, é separável e consequentemente $W$ é um espaço de Banach separável. Do fato de que $B_{1}^{W^{*}}(0)$ é compacto e metrizável na topologia fraca* $\sigma\left(W^{*}, W\right)$, segue que existe uma sequência densa $\left\{\phi_{n}\right\}_{n \in \mathbb{N}}$ em $\left(B_{1}^{W^{*}}(0), \sigma\left(W^{*}, W\right)\right)$. Agora se $x \in W$ e $\phi_{n}(x)=0$ para todo $n \in N$, segue que $\phi(x)=0$ para todo $\phi \in B_{1}^{W^{*}}(0)$ e temos que $x=0$. A sequência desejada é obtida estendendo $\phi_{i}$ a $X$ através do Teorema de Hahn-Banach, para cada $n \in \mathbb{N}$.

Teorema 3.2.5 (Mañé [17], Lema 1.1). Se $c(K)<\infty$ e $Y$ é um subespaço de dimensão finita de $X \operatorname{com} 2 c(K)+1<\operatorname{dim} Y<\infty$, então o conjunto $\{P \in \mathcal{P}(X, Y)$ : $\left.P\right|_{K}$ é injetora $\}$ é residual em $\mathcal{P}(X, Y)$.

Demonstração: Usando a definição de $P_{n, r}$ dado no Lema 3.2.2, não é difícil ver que $\cap_{n \in \mathbb{N}} \cap_{m \in \mathbb{N}} P_{n, \frac{1}{m}}$ é o conjunto das projeções em $\mathcal{P}(X, Y)$ que são injetivas em $K$. Para concluir a prova do teorema precisamos somente mostrar que $P_{n, r}$ é denso em $\mathcal{P}(X, Y)$ para cada $n \in \mathbb{N}$ e para cada $r>0$, e o resultado seguirá do Teorema de Baire. 
Seja $Q$ a aplicação quociente de $X$ sobre $Z=X / Y$. Então,

$$
Q\left(A_{n, r}\right) \backslash\{0\}=\bigcup_{m \in \mathbb{N}}\left\{Q(v): v \in A_{n, r},\|Q(v)\|_{Z} \geqslant \frac{1}{m}\right\}
$$

onde, cada $\left\{Q(v): v \in A_{n, r},\|Q(v)\|_{Z} \geqslant \frac{1}{m}\right\}$ é compacto. Segue do Lema 3.2.4 que existe uma sequência $\left\{\phi_{i}\right\}_{i \in \mathbb{N}}$ em $Z^{*}, \phi_{i}: Z \rightarrow \mathbb{R}$, tal que, se $\phi_{i}(z)=0$ para todo $i \in \mathbb{N}$, então $z=0$.

Seja

$$
A_{n, r, i, j}=\left\{v \in A_{n, r}:\left|\phi_{i}(Q(v))\right| \geqslant 1 / j\right\} .
$$

Então

$$
\begin{gathered}
P^{-1}(0) \cap A_{n, r}=\cup_{i \in \mathbb{N}} \cup_{j \in \mathbb{N}}\left(P^{-1}(0) \cap A_{n, r, i, j}\right), \\
P_{n, r, i, j}=\left\{P \in \mathcal{P}(X, Y): P^{-1}(0) \cap A_{n, r, i, j}=\emptyset\right\}
\end{gathered}
$$

e

$$
P_{n, r}=\bigcap_{i \in \mathbb{N}} \cap_{j \in \mathbb{N}} P_{n, r, i, j}
$$

Como $A_{n, r}$ é compacto (vide Lema 3.2.1) segue facilmente que $A_{n, r, i, j}$ é compacto. Procedendo exatamente como na demonstração do Lema 3.2 .3 obtemos que $P_{n, r, i, j}$ é aberto. Portanto, a prova fica reduzida a mostrar que para cada $n, i, j \in \mathbb{N}$ e $r>0, P_{n, r, i, j}$ é denso em $\mathcal{P}(X, Y)$.

Seja $P_{0} \in \mathcal{P}(X, Y)$ e defina $\phi: Y \backslash\{0\} \rightarrow S=\left\{y \in Y:\|y\|_{X}=1\right\}$ por $\phi(y)=y /\|y\|_{X}$. Então

$$
\phi\left(P_{0}\left(A_{n, r}\right) \backslash\{0\}\right)=\cup_{\ell \in \mathbb{N}} \phi\left(P_{0}\left(A_{n, r}\right) \cap\left[Y \backslash B_{1 / \ell}^{Y}(0)\right]\right)
$$

e, da Proposição 3.1 .10 ,

$$
\operatorname{dim}_{H}\left(\phi\left(P_{0}\left(A_{n, r}\right)\right)\right) \leqslant \sup _{\ell \in \mathbb{N}} \operatorname{dim}_{H}\left(\phi\left(P_{0}\left(A_{n, r}\right) \cap\left[Y \backslash B_{1 / \ell}^{Y}(0)\right]\right)\right) .
$$

Note que, $\phi$ restrito à $P_{0}\left(A_{n, r}\right) \cap\left[Y \backslash B_{1 / \ell}^{Y}(0)\right]$ é Lipschitz contínua. Consequentemente

$$
\operatorname{dim}_{H}\left(\phi\left(P_{0}\left(A_{n, r}\right) \cap\left[Y \backslash B_{1 / \ell}^{Y}(0)\right]\right)\right) \leqslant \operatorname{dim}_{H}\left(P_{0}\left(A_{n, r}\right)\right) .
$$


Portanto, usando o Corolário 3.1 .12 temos que

$$
\left.\operatorname{dim}_{H}\left(\phi\left(P_{0}\left(A_{n, r}\right)\right)\right) \leqslant \operatorname{dim}_{H}\left(P_{0}\left(A_{n, r}\right)\right)\right) \leqslant \operatorname{dim}_{H}\left(A_{n, r}\right) \leqslant 2 c\left(K_{n}\right) \leqslant 2 c(K) .
$$

Disto obtemos que existe $u \in S \backslash \phi\left(P_{0}\left(A_{n, r}\right)\right)$. De fato, se este não é o caso, então $S \subset \phi\left(P_{0}\left(A_{n, r}\right)\right)$ e $\operatorname{dim}(Y)-1=\operatorname{dim}_{H}(S) \leqslant \operatorname{dim}_{H}\left(\phi\left(P_{0}\left(A_{n, r}\right)\right)\right) \leqslant 2 c(K)$ o que contradiz a nossa hipótese.

Dado $\epsilon>0, i, j \in \mathbb{N}$ definimos

$$
P_{\epsilon}(x)=P_{0}(x)+\epsilon \phi_{i}(Q(x)) u
$$

Como $P_{\epsilon} \in \mathcal{L}(X)$ com imagem em $Y$ e relembrando que se $y \in Y$, então $Q y=0$ é fácil ver que $P_{\epsilon} \in \mathcal{P}(X, Y)$.

Se $P_{\epsilon}(x)=0$ temos que

$$
P_{0}(x)=-\epsilon \phi_{i}(Q(x)) u
$$

Se em adição $x \in A_{n, r, i, j}$ temos que $\phi_{i}(Q(x)) \neq 0$. Portanto

$$
u=-\left(\epsilon \phi_{i}(Q(x))\right)^{-1} P_{0}(x)
$$

Como $u \in S, u=\phi(u)$ e assim $\phi\left(P_{0}(x)\right)=P_{0}(x)$, o que nos dá $\left|\epsilon \phi_{i}(Q(x))\right|=1$ e portanto $\pm u=\phi\left(P_{0}(x)\right) \in \phi\left(P_{0}\left(A_{n, r, i, j}\right)\right)$. Consequentemente $u \in \phi\left(P_{0}\left(A_{n, r}\right)\right)$ contradizendo a escolha de $u$ e mostrando que $P_{\epsilon} \in P_{n, r, i, j}$.

Como $\left\|P_{\epsilon}-P_{0}\right\|_{\mathcal{L}(X)} \stackrel{\epsilon \rightarrow 0}{\longrightarrow} 0$ temos que $P_{n, r, i, j}$ é denso em $\mathcal{P}(X, Y)$.

\subsection{Dimensão de conjuntos compactos negativamente in-}

\section{variantes}

Exploramos na seção anterior a projeção de conjuntos compactos com dimensão fractal finita em espaços vetoriais de dimensão finita de maneira injetiva. Para concluir o nosso estudo sobre atratores, resta-nos mostrar que estes têm de fato dimensão fractal finita, o que nos mostrará que eles são de fato objetos de dimensão finita.

Nesta seção, nos dedicamos a apresentar, corrigir e melhorar os resultados apresentados em Mañé [17] e em Hale [12] sobre dimensão de conjuntos compactos negativamente 
invariantes.

Seja $X_{1}, X_{2}$ um espaço de Banach e defina

$$
\mathcal{L}_{\lambda}\left(X_{1}, X_{2}\right)=\left\{T \in \mathcal{L}\left(X_{1}, X_{2}\right): T=L+C \text { com } C \text { compacto e }\|L\|_{\mathcal{L}\left(X_{1}, X_{2}\right)}<\lambda\right\} .
$$

Para prosseguirmos, introduziremos a noção de distância de Banach-Mazur entre dois espaços normados isomorfos e enunciaremos um resultado que melhora a estimativa obtida por Mañé, seguindo Carvalho-Langa-Robinson [6].

Definição 3.3.1. Sejam $X$ e $Y$ dois espaços normados isomorfos. Definimos a distância de Banach-Mazur entre $X$ e $Y$ por

$$
\mathrm{d}_{B M}(X, Y)=\log \left(\inf \left\{\|T\|_{\mathcal{L}(X, Y)}\left\|T^{-1}\right\|_{\mathcal{L}(Y, X)}: T \in \mathcal{L}(X, Y), T^{-1} \in \mathcal{L}(Y, X)\right\}\right)
$$

Podemos ver facilmente que $\mathrm{d}_{B M}(X, Y)=0$ se, e somente se, $X$ e $Y$ são isometricamente isomorfos.

Denotemos também $\mathbb{K}_{\infty}^{m}$ o espaço $\mathbb{K}^{m}$ munido da norma $\ell_{\infty}(\mathbb{K}=\mathbb{R}$ ou $\mathbb{C})$; isto é, para $\mathbf{z} \in \mathbb{K}^{m}$ com $\mathbf{z}=\left(z_{1}, \cdots, z_{m}\right)$, onde $z_{i} \in \mathbb{K}$ temos

$$
\|\mathbf{z}\|_{\infty}=\max _{i=1, \cdots, m}\left\|z_{i}\right\|_{\mathbb{K}}
$$

Nosso objetivo é realizar a estimativa $\mathrm{d}_{B M}\left(X, \mathbb{K}_{\infty}^{m}\right) \leqslant \log m$, onde $X$ é um espaço de Banach $m$-dimensional. Para isto faremos uso de uma base de Auerbach para $X$. Vamos então estabelecer a existência de tais bases para espaços reais, e depois para espaços complexos.

Lema 3.3.2. Seja $X$ um espaço normado real $n$-dimensional. Então, existe uma base $B=\left\{x_{1}, \cdots, x_{n}\right\}$ para $X$ e uma base $B^{*}=\left\{x_{1}^{*}, \cdots, x_{n}^{*}\right\}$ para $X^{*}$ com $\left\|x_{i}\right\|_{X}=\left\|x_{i}^{*}\right\|_{X^{*}}=$ 1, e $x_{i}^{*}\left(x_{j}\right)=\delta_{i j}, 1 \leqslant i, j \leqslant n$.

Demonstração: Se $X=\left(\mathbb{R}^{n},\|\cdot\|\right)$, tome vetores $\left\{x_{1}, \cdots, x_{n}\right\}$ com $\left\|x_{i}\right\|_{X}=1,1 \leqslant i \leqslant n$ e tal que a envoltória convexa fechada $\overline{c o}\left\{0, x_{1}, \cdots, x_{n}\right\}$ tenha volume máximo. Defina $x_{i}^{*}$ tal que $x_{i}^{*}\left(x_{j}\right)=\delta_{i j}, 1 \leqslant j \leqslant n$ e $1 \leqslant i \leqslant n$. Resta somente provar que $\left\|x_{i}^{*}\right\|_{X^{*}}=1$, $1 \leqslant i \leqslant n$. Claramente $\left\|x_{i}^{*}\right\|_{X^{*}} \geqslant 1$.

Se $\left\|x_{i}^{*}\right\|_{X^{*}}>1$, seja $y_{i} \in X$ com $\left\|y_{i}\right\|_{X}=1$ tal que $x_{i}^{*}\left(y_{i}\right)>1$. Note que $\{x \in$ $\left.\mathbb{R}^{n}: x_{i}^{*}(x)=1\right\}$ define um hiperplano paralelo ao hiperplano $\left\{x \in \mathbb{R}^{n}: x_{i}^{*}(x)=0\right\}=$ 
$\operatorname{span}\left\{x_{1}, \cdots, x_{i-1}, x_{i+1}, x_{n}\right\}$. Portanto $\overline{c o}\left\{0, x_{1}, \cdots, x_{n}\right\}$ tem volume estritamente menor que $\overline{c o}\left\{0, x_{1}, \cdots, x_{i-1}, y_{i}, x_{i+1}, \cdots, x_{n}\right\}$ e temos uma contradição.

Lema 3.3.3. Seja X um espaço vetorial complexo normado n-dimensional. Então, existe uma base $B=\left\{x_{1}, \cdots, x_{n}\right\}$ para $X$ e uma base $B^{*}=\left\{f_{1}, \cdots, f_{n}\right\}$ para $X^{*}$ com $\left\|x_{i}\right\|_{X}=$ $\left\|f_{i}\right\|_{X^{*}}=1(i=1, \ldots, n)$ tal que $f_{i}\left(x_{j}\right)=\delta_{i j}, i, j=1, \ldots, n$.

Demonstração: Dada uma base $B_{0}=\left\{y_{1}, \cdots, y_{n}\right\}$ de $X$ consideramos o espaço real $X_{2}$ dado pela combinações lineares sobre $\mathbb{R}$ de

$$
B_{2}=\left\{y_{1}, \ldots, y_{n}, \mathrm{i} y_{1}, \ldots, \mathrm{i} y_{n}\right\}
$$

equipado com a norma $\|z\|_{X_{2}}=\|z\|_{X}$. Aplicamos agora a versão real deste resultado para $X_{2}$, para produzir uma base $\left\{x_{1}, \ldots, x_{2 n}\right\}$ para $X_{2}$ e $\left\{\varphi_{1}, \ldots, \varphi_{2 n}\right\}$ para $X_{2}^{*}$ tal que $\varphi_{j}\left(x_{k}\right)=\delta_{j k}$ e $\left\|\varphi_{j}\right\|=\left\|x_{j}\right\|=1$.

Como $x_{j} \in X$ e $\left\{x_{j}\right\}$ deve gerar $X$ sobre $\mathbb{C}$, podemos reordenar e renomear os $\left\{x_{j}\right\}$ (e as correspondentes $\left.\left\{\varphi_{j}\right\}\right)$ de forma que $\left\{x_{1}, \ldots, x_{n}\right\}$ gerem $X$ sobre $\mathbb{C}$. Segue que

$$
\left\{x_{1}, \ldots, x_{n}, \mathrm{i} x_{1}, \ldots, \mathrm{i} x_{n}\right\}
$$

gera $X$ sobre $\mathbb{R}$. Mais ainda, cada i $x_{j}$ deve ser uma combinação linear de $\left\{x_{n+1}, \ldots, x_{2 n}\right\}$, como segue do fato de que $\left\{x_{1}, \ldots, x_{n}\right\}$ forma uma base para $X$ sobre $\mathbb{C}$ que $\left\{\mathrm{i} x_{1}, x_{2}, x_{n}\right\}$ são linearmente independentes (sobre $\mathbb{C}$, logo certamente sobre $\mathbb{R}$ ).

Para $k=1, \ldots, n$ definimos um elemento $f_{k} \in X^{*}$ por

$$
f_{k}(z)=\varphi_{k}(z)-\mathrm{i} \varphi_{k}(\mathrm{i} z) \quad z \in X
$$

onde para interpretar $\varphi_{k}(z)$ consideramos $z$ como um elemento de $X_{2}$ (basta expandir em termos da base $\left\{x_{1}, \ldots, x_{n}\right\}$ e separar as partes reais e imaginárias dos coeficientes). Mostremos agora que $\left\{f_{k}\right\}$ tem as propriedades desejadas.

Primeiramente, notemos que para $1 \leqslant k, j \leqslant n$,

$$
f_{k}\left(x_{j}\right)=\varphi_{k}\left(x_{j}\right)-\mathrm{i} \varphi_{k}\left(\mathrm{i} x_{j}\right)=\delta_{k j},
$$

como i $x_{j}$ é uma combinação linear de $\left\{x_{n+1}, \ldots, x_{2 n}\right\}$, e portanto $\varphi_{k}\left(\mathrm{i} x_{j}\right)=0$. Tudo que nos resta provar é que $\left\|x_{k}^{*}\right\|=1$. Para isto seguimos um argumento padrão (por exemplo, 
Yosida, 1980), escrevendo $f_{k}(z)=r e^{-\mathrm{i} \theta}$. Então

$$
\left|f_{k}(z)\right|=e^{\mathrm{i} \theta} f_{k}(z)=f_{k}\left(e^{\mathrm{i} \theta} z\right)
$$

$\operatorname{logo} f_{k}\left(e^{\mathrm{i} \theta} z\right)$ é real e positivo. Segue que

$$
\left|f_{k}(z)\right|=f_{k}\left(e^{\mathrm{i} \theta} z\right)=\varphi_{k}\left(e^{\mathrm{i} \theta} z\right) \leq\left\|e^{\mathrm{i} \theta} z\right\|_{X_{2}} \leq\left\|e^{\mathrm{i} \theta} z\right\|_{X}=\|x\|_{X}
$$

e o lema está provado.

Agora, com a existência da base de Auerbach para espaços de dimensão finita, podemos prosseguir com um importante resultado sobre a distância de Banach-Mazur, que é uma melhora na estimativa feita por Mañé, que afirma que, se $Y$ é um espaço de Banach $m$-dimensional sobre $\mathbb{K}=\mathbb{R}$ ou $\mathbb{C}$, então

$$
\mathrm{d}_{B M}\left(Y, \mathbb{K}_{\infty}^{m}\right) \leqslant \log \left(m 2^{m}\right)
$$

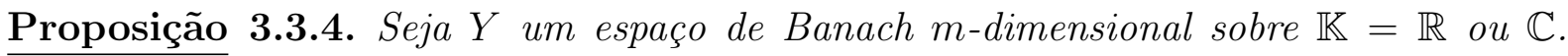
$\operatorname{Então} \mathrm{d}_{B M}\left(Y, \mathbb{K}_{\infty}^{m}\right) \leqslant \log m$.

Demonstração: Seja $\left\{x_{1}, \cdots, x_{m}\right\}$ uma base de Auerbach para $Y$, e $\left\{f_{1}, \cdots, f_{m}\right\}$ a base correspondente de $Y^{*}$. Defina a aplicação $T: \mathbb{K}_{\infty}^{m} \rightarrow Y$ da seguinte maneira

$$
T(\mathbf{z})=\sum_{j=1}^{m} z_{j} x_{j}
$$

Então

$$
\|T(\mathbf{z})\|_{X}=\left\|\sum_{j=1}^{m} z_{j} x_{j}\right\|_{Y} \leq \sum_{j=1}^{m}\left|z_{j}\right| \leqslant m\|\mathbf{z}\|_{\infty},
$$

e portanto

$$
\|T\|_{\mathcal{L}\left(\mathbb{K}_{\infty}^{m}, Y\right)} \leqslant m
$$

Por outro lado, se $x=\sum_{j=1}^{m} z_{j} x_{j} \in Y$ com $\|x\|_{Y} \leqslant 1$ então como $z_{j}=f_{j}(x)$,

$$
\left\|T^{-1}(x)\right\|_{\infty}=\|\mathbf{z}\|_{\infty}=\max _{j=1, \ldots, m}\left|z_{j}\right|=\max _{j=1, \ldots, m}\left|f_{j}(x)\right| \leqslant\|x\|_{Y},
$$


o que implica que

$$
\left\|T^{-1}\right\|_{\mathcal{L}\left(Y, \mathbb{K}_{\infty}^{m}\right)} \leqslant 1
$$

e demonstra nosso resultado.

Lema 3.3.5. Seja $X$ um espaço de Banach sobre $\mathbb{K}$. Se $Y$ é um subespaço de $X$ e $\operatorname{dim}(Y)=m$, temos

$$
N\left(\rho, B_{r}^{Y}(0)\right) \leqslant(m+1)^{\alpha m}\left(\frac{r}{\rho}\right)^{\alpha m}, 0<\rho \leqslant r
$$

onde $\alpha=1$ se $\mathbb{K}=\mathbb{R}$ e $\alpha=2$ se $\mathbb{K}=\mathbb{C}$. As bolas na cobertura podem ser tomadas com centro em $Y$.

Demonstração: Assuma primeiramente que $\mathbb{K}=\mathbb{R}$. Como $Y$ e $\mathbb{R}_{\infty}^{m}$ são $m$-dimensionais, $\mathrm{d}_{B M}\left(Y, \mathbb{R}_{\infty}^{m}\right) \leqslant \log m$; em particular, existe um isomorfismo linear $T: \mathbb{R}_{\infty}^{m} \rightarrow Y$ tal que $\|T\|\|T\|^{-1} \leqslant m$. Como

$$
B_{r}^{Y}(0)=T T^{-1}\left(B_{r}^{Y}(0)\right) \subseteq T\left(B_{\left\|T^{-1}\right\| r}^{\mathbb{R}^{m}}(0)\right)
$$

e $B_{\left\|T^{-1}\right\| r}^{\mathbb{R}_{\infty}^{m}}(0)$ pode ser coberta por

$$
\left(1+\frac{\left\|T^{-1}\right\| r}{\rho /\|T\|}\right)^{m}=\left(1+\|T\|\left\|T^{-1}\right\| \frac{r}{\rho}\right)^{m} \leq\left(1+m \frac{r}{\rho}\right)^{m} \leqslant(m+1)^{m}\left(\frac{r}{\rho}\right)^{m}
$$

bolas em $\mathbb{R}_{\infty}^{m}$ de raio $\rho /\|T\|$, segue que $B_{r}^{Y}(0)$ pode ser coberta pelo mesmo número de $Y$-bolas de raio $\rho$. Se $X$ é complexo são necessárias $(1+(a / b))^{2 m}$ bolas de raio $b$ em $\mathbb{C}_{\infty}^{m}$ para cobrir uma bola de raio $a$.

Antes de continuarmos precisamos do seguinte Lema:

Lema 3.3.6. Seja $X$ um espaço de Banach e $T \in \mathscr{L}_{\lambda / 2}(X)$. Então existe um subespaço $Z$ de $X$ de dimensão finita tal que

$$
\operatorname{dist}\left(T\left[B_{1}^{X}(0)\right], T\left[B_{1}^{Z}(0)\right]\right)<\lambda .
$$

Denotamos por $\nu_{\lambda}(T)$ o minimo $n \in \mathbb{N}$ tal que 3.3.1 vale para algum subespaço $n$ dimensional de $X$. 
Demonstração: $\quad$ Escreva $T=L+C$, onde $C \in \mathcal{K}(X)$ and $L \in \mathcal{L}(X) \operatorname{com}\|L\|_{\mathcal{L}(X)}<\lambda / 2$. Mostremos primeiramente que para qualquer $\epsilon>0$ existe um subespaço de dimensão finita $Z$ tal que

$$
\operatorname{dist}\left(C\left[B_{1}^{X}(0)\right], C\left[B_{1}^{Z}(0)\right]\right)<\epsilon .
$$

Suponha que este não é o caso. Escolha algum $x_{1} \in X \operatorname{com}\left\|x_{1}\right\|_{X}=1$, e seja $Z_{1}=$ $\operatorname{span}\left\{x_{1}\right\}$. Então

$$
\operatorname{dist}\left(C\left[B_{1}^{X}(0)\right], C\left[B_{1}^{Z_{1}}(0)\right]\right) \geqslant \epsilon
$$

e logo existe um $x_{2} \in X$ com $\left\|x_{2}\right\|_{X}=1$ tal que

$$
\left\|C x_{2}-C x_{1}\right\|_{X} \geqslant \epsilon
$$

Com $Z_{2}=\operatorname{span}\left\{x_{1}, x_{2}\right\}$, podemos encontrar $x_{3}$ com $\left\|x_{3}\right\|_{X}=1$ tal que

$$
\left\|C x_{3}-C x_{1}\right\|_{X} \geqslant \epsilon \text { e }\left\|C x_{3}-C x_{2}\right\|_{X} \geqslant \epsilon .
$$

Continuando indutivamente podemos construir desta maneira uma sequência $\left\{x_{j}\right\}$ com $\left\|x_{j}\right\|=1$ tal que

$$
\left\|C x_{i}-C x_{j}\right\|_{X} \geqslant \epsilon \quad i \neq j
$$

contradizendo a compacidade de $C$.

Agora seja $\tilde{\lambda}<\lambda$ tal que $2\|L\|_{\mathcal{L}(X)}<\tilde{\lambda}<\lambda$, e escolha $Z$ usando o argumento acima de forma que

$$
\operatorname{dist}\left(C\left[B_{1}^{X}(0)\right], C\left[B_{1}^{Z}(0)\right]\right)<\lambda-\tilde{\lambda} .
$$

Se $x \in B_{1}^{X}(0)$ e $z \in B_{1}^{Z}(0)$, então

$$
\|T x-T z\|_{X} \leqslant\|L(x-z)\|_{X}+\|C x-C z\|_{X} \leqslant \tilde{\lambda}+\|C x-C z\|_{X}
$$

Portanto,

$$
\begin{aligned}
\operatorname{dist}\left(T\left[B_{1}^{X}(0)\right], T\left[B_{1}^{Z}(0)\right]\right) & \leqslant \tilde{\lambda}+\operatorname{dist}\left(C\left[B_{1}^{X}(0)\right], C\left[B_{1}^{Z}(0)\right]\right) \\
& <\lambda
\end{aligned}
$$

Isto completa a demonstração. 
Lema 3.3.7. Se $T \in \mathcal{L}(X)$ e $Y \subset X$ é um subespaço com $\operatorname{dim}(Y)=m$, então

$$
N\left((1+\gamma) \lambda r, T\left(B_{r}^{X}(0)\right)\right) \leqslant(m+1)^{\alpha m}\left(\frac{\|T\|_{\mathcal{L}(X)}+\lambda}{\gamma \lambda}\right)^{\alpha m}
$$

para todo $r>0, \lambda>\operatorname{dist}\left(T\left[B_{1}^{X}(0)\right], T\left[B_{1}^{Y}(0)\right]\right), \gamma>0$, onde $\alpha=1$ (ou 2) se $X$ é real (ou complexo).

Demonstração: Pela linearidade de $T$ é suficiente mostrar o teorema para $r=1$. Seja $\bar{r}=\|T\|_{\mathcal{L}(X)}+\lambda$. Cubra a bola $B_{\bar{r}}(0) \cap T(Y)$ por bolas $B_{\gamma \lambda}\left(x_{i}\right), 1 \leqslant i \leqslant k, \operatorname{com} x_{i} \in B_{\bar{r}}(0)$ para todo $i$. Pelo Lema 3.3 .5 podemos tomar

$$
k \leqslant(m+1)^{\alpha m}\left(\frac{\bar{r}}{\gamma \lambda}\right)^{\alpha m}
$$

A demonstração estará completa se mostrarmos que

$$
\bigcup_{i=1}^{k} B_{(1+\gamma) \lambda}\left(x_{i}\right) \supset T\left(B_{1}(0)\right) .
$$

Se $\|v\|_{X} \leqslant 1$, como $\operatorname{dist}\left(T\left[B_{1}^{X}(0)\right], T\left[B_{1}^{Y}(0)\right]\right)<\lambda$, existe $y \in B_{1}^{Y}(0)$ tal que

$$
\|T v-T y\|_{X}<\lambda
$$

Chamando $v_{2}=T y$ temos

$$
\left\|v_{2}\right\|_{X} \leqslant\|T v\|_{X}+\left\|T v-v_{2}\right\|_{X} \leqslant\|L\|_{\mathcal{T}(X)}\|v\|_{X}+\lambda=\bar{r} .
$$

Escolhendo $1 \leqslant i \leqslant k$ tal que $\left\|v_{2}-x_{i}\right\|_{X} \leqslant \gamma \lambda$ temos que

$$
\left\|T v-x_{i}\right\|_{X} \leqslant\left\|T v-v_{2}\right\|_{X}+\left\|v_{2}-x_{i}\right\|_{X} \leqslant \lambda(1+\gamma) .
$$

Isto completa a demonstração.

Lema 3.3.8. Sejam $K$ um subconjunto compacto de um espaço de Banach $X$ e $f: X \rightarrow X$ uma função continuamente diferenciável em uma vizinhança de $K$. Suponha que $K$ seja negativamente invariante para $f$; isto é, $f(K) \supset K$, e suponha também que existam 
$0<\alpha<1$ e $M \geqslant 1$ tal que para cada $x \in K$,

$$
N\left(\alpha, D_{x} f\left[B_{1}^{X}(0)\right]\right) \leq M
$$

Então

$$
c(K) \leq \frac{\log M}{-\log \alpha}
$$

Demonstração: Primeiramente, garantimos que (3.3.3) é suficiente para fornecer limitações para o número de bolas necessárias para cobrir $f\left(B_{r}^{X}(x)\right)$ quando $r$ é suficientemente pequeno. Como $f$ é continuamente diferenciável e $K$ é compacto, para cada $\eta>0$ existe $r_{0}=r_{0}(\eta)$ tal que para qualquer $0<r<r_{0}$ e qualquer $x \in K$,

$$
f\left(B_{r}^{X}(x)\right) \subseteq f(x)+D_{x} f\left[B_{r}^{X}(0)\right]+B_{\eta r}^{X}(0) .
$$

Segue que

$$
N\left((\alpha+\eta) r, f\left[B_{r}^{X}(x)\right]\right) \leqslant M
$$

para todo $r \leq r_{0}(\eta)$.

Agora fixe $\eta$ com $0<\eta<1-\alpha$, e seja $r_{0}=r_{0}(\eta)$. Cubra $K \operatorname{com} N\left(r_{0}, K\right)$ bolas de raio $r_{0}$. Aplique $f$ para todo elemento desta cobertura. Como $f(K) \supseteq K$, isto nos fornece uma cobertura de $K$ formada por conjuntos da forma $f\left(B_{X}\left(x, r_{0}\right)\right)$, para algum $x \in K$. Segue de 3.3.5 que cada uma destas imagens pode ser coberta por $M$ bolas de raio $(\alpha+\eta) r_{0}$, garantindo que $N\left((\alpha+\eta) r_{0}, K\right) \leqslant M N\left(r_{0}, K\right)$. Aplicando este argumento $k$ vezes, temos

$$
N\left((\alpha+\eta)^{k} r_{0}, K\right) \leq M^{k} N\left(r_{0}, K\right)
$$

Segue da definição de $c(K)$ que

$$
c(K) \leq \frac{\log M}{-\log (\alpha+\eta)},
$$

e como $\eta>0$ é arbitrário obtemos (3.3.4.

Teorema 3.3.9. Seja $X$ um espaço de Banach, $U \subset X$ um conjunto aberto e $f: U \rightarrow$ $X$ uma aplicação continuamente diferenciável. Suponha que $K \subset U$ é um subconjunto compacto e que $D_{x} f \in \mathcal{L}_{\frac{\lambda}{2}}(X)$, para algum $0<\lambda<\frac{1}{2}$, para todo $x \in K$. Então $n=$ 
$\sup _{x \in K} \nu_{\lambda}\left(D_{x} f\right)$ e $D=\sup _{x \in K}\left\|D_{x} f\right\|$ são finitos $e$

$$
N\left(2 \lambda, D_{x} f\left[B_{1}^{X}(0)\right]\right) \leqslant\left[(n+1) \frac{D}{\lambda}\right]^{\alpha n} \quad \text { para todo } \quad x \in K,
$$

onde $\alpha=1$ se $X$ é real e $\alpha=2$ se $X$ é complexo. Se ainda $f(K) \supset K$ então

$$
c(K) \leqslant \alpha n\left\{\frac{\log ((n+1) D / \lambda)}{-\log (2 \lambda)}\right\}
$$

Demonstração: Primeiramente mostremos que $n=\sup _{x \in K} \nu_{\lambda}\left(D_{x} f\right)$ é finito. Para cada $x \in K$, existe um subespaço linear de dimensão finita $Z_{x}$ tal que

$$
\operatorname{dist}\left(D_{x} f\left[B_{1}^{X}(0)\right], D_{x} f\left[B_{1}^{Z_{x}}(0)\right]\right)<\lambda
$$

Como $D_{(\cdot)} f$ é contínua, segue que existe um $\delta_{x}>0$ tal que

$$
\operatorname{dist}\left(D_{y} f\left[B_{1}^{X}(0)\right], D_{y} f\left[B_{1}^{Z_{x}}(0)\right]\right)<\lambda
$$

para todo $y \in B_{X}\left(x, \delta_{x}\right)$, isto é, $\nu_{\lambda}(y) \leqslant \nu_{\lambda}(x)$ para estes tais $y$. A cobertura aberta de $K$ formada pela união de $B_{\delta_{x}}^{X}(x)$ sobre $x$ tem uma subcobertura finita, donde segue que $n<\infty$.

Agora, como $n=\sup _{x \in K} \nu_{\lambda}\left(D_{x} f\right)<\infty$, para cada $x \in K$ existe um subespaço $Z_{x}$ de $X \operatorname{com} \operatorname{dim}\left(Z_{x}\right) \leqslant n$ tal que

$$
\operatorname{dist}\left(D_{x} f\left[B_{1}^{X}(0)\right], D_{x} f\left[B_{1}^{Z_{x}}(0)\right]\right)<\lambda
$$

Para facilitar a notação vamos omitir o subscrito $x$ em $Z_{x}$, e escreveremos $T=D_{x} f$.

Notando que $T(Z)$ é também um subespaço $n$-dimensional de $X$, podemos usar o Lemma 3.3 .5 para cobrir a bola $B_{\|T\|}^{T(Z)}(0)$ com bolas $B_{\lambda}^{X}\left(y_{i}\right), 1 \leqslant i \leqslant k$, tal que $y_{i} \in B_{\|T\|}^{X}(0)$ para cada $i$ e

$$
k \leqslant\left[(n+1) \frac{\|T\|}{\lambda}\right]^{\alpha n} .
$$

Logo

$$
T\left[B_{1}^{Z}(0)\right] \subseteq B_{\|T\|}^{T(Z)}(0)=B_{\|T\|}^{X}(0) \cap T(Z) \subseteq \bigcup_{i=1}^{k} B_{\lambda}^{X}\left(y_{i}\right)
$$


Completaremos a prova mostrando que

$$
\bigcup_{i=1}^{k} B_{2 \lambda}^{X}\left(y_{i}\right) \supseteq T\left[B_{1}^{X}(0)\right] .
$$

De fato, se $x \in B_{X}(0,1)$ então segue de 3.3.1) que existe um $y \in T\left[B_{1}^{Z}(0)\right]$ tal que $\|T x-y\|_{X}<\lambda$. Como $y \in T\left[B_{1}^{Z}(0)\right]$, segue de 3.3.8) que $\left\|y-y_{i}\right\|_{X} \leqslant \lambda$ para algum $i \in\{1, \ldots, k\}$, e $\log \mathrm{O}$

$$
\left\|T x-y_{i}\right\|_{X} \leqslant\|T x-y\|_{X}+\left\|y-x_{i}\right\|_{X}<2 \lambda,
$$

isto é, $x \in B_{2 \lambda}^{X}\left(y_{i}\right)$.

O resultado segue como enunciado, uma vez que $n$ é uniforme sobre $x \in K$. A conclusão final segue do Lema 3.3 .8 .

Teorema 3.3.10. Seja $X$ um espaço de Banach, $U \subset X$ um conjunto aberto e $f: U \rightarrow X$ uma aplicação continuamente diferenciável. Se $K \subset U$ é um conjunto compacto tal que $f(K) \supset K$ e que existe $\epsilon>0$ tal que $D_{x} f \in \mathcal{L}_{1-\epsilon}(X)$ para todo $x \in K$, então

$$
c(K)<\infty
$$

Se em adição $D_{x} f \in \mathcal{L}_{\frac{1}{4}}(X)$ para todo $x \in K$ temos que

$$
c(K) \leqslant \frac{\log \left[(\nu+1)^{\alpha \nu}\left(\frac{D+\lambda}{\epsilon \lambda}\right)^{\alpha \nu}\right]}{\log (1 / 2(1+\epsilon) \lambda)}
$$

onde $D=\sup _{x \in K}\left\|D_{x} f\right\|, \nu=\sup _{x \in K} \nu_{\lambda}\left(D_{x} f\right), 0<\lambda<\frac{1}{2}$ e $\epsilon$ é tal que $(1+\epsilon) \lambda<1 / 2$.

Demonstração: Primeiramente notemos que, se definirmos $\tilde{N}(r, K)$ como o número mínimo de bolas $B_{r}(x), x \in X$ (e não $x \in K$ como na definição de $N(r, K)$ ) necessário para cobrir $K$, então

$$
N(2 r, K) \leqslant \tilde{N}(r, K) \leqslant N(r, K) .
$$


Portanto

$$
\begin{aligned}
c(K) & =\limsup _{r \rightarrow 0} \frac{\log N(2 r, K)}{\log (1 / 2 r)}=\limsup _{r \rightarrow 0} \frac{\log N(2 r, K)}{\log (1 / r)} \\
& \leqslant \limsup _{r \rightarrow 0} \frac{\log \tilde{N}(r, K)}{\log (1 / r)} \leqslant \limsup _{r \rightarrow 0} \frac{\log N(r, K)}{\log (1 / r)}=c(K) .
\end{aligned}
$$

e

$$
\limsup _{r \rightarrow 0} \frac{\log \tilde{N}(r, K)}{\log (1 / r)}=c(K)
$$

Como, para cada $y \in K$ temos que $D_{y} f \in \mathcal{L}_{1}(X)$; isto é $D_{y} f=L_{y}+C_{y}$ e como

$$
D_{x} f^{n}=D_{f^{n-1}(x)} f \circ D_{f^{n-2}(x)} f \circ \cdots \circ D_{x} f=L+C
$$

onde $D_{f^{n-j}(x)} f=L_{j}+C_{j}, L=L_{1} \circ \cdots \circ L_{n} \operatorname{com} L_{j} \in \mathcal{L}_{1-\epsilon}(X), C_{j} \in \mathcal{K}(X), 1 \leqslant j \leqslant n$, e $C \in K(X)$.

Segue que, para $n$ suficientemente grande, $g=f^{n}$ é tal que $D_{x} g \in \mathcal{L}_{\frac{\lambda}{2}}(X)$ para todo $x \in K$ e algum $0<\lambda<1 / 2$. Do Lema 3.3.6, para cada $x \in K$ existe um subespaço $Z_{x}$ de $X \operatorname{com} \operatorname{dim}\left(Z_{x}\right) \leqslant \nu$ tal que $\operatorname{dist}\left(D_{x} g\left[B_{1}^{X}(0)\right], D_{x} g\left[B_{1}^{Z_{x}}(0)\right]\right)<\lambda$. Agora, do Lema 3.3.7 temos que para cada $r>0$ e $\frac{1}{2 \lambda}-1>\epsilon>0$,

$$
\bar{N}_{\epsilon}:=N\left((1+\epsilon) \lambda r, D_{x} g\left(B_{r}(0)\right)\right) \leqslant(\nu+1)^{\alpha \nu}\left(\frac{\left\|D_{x} g\right\|_{\mathcal{L}(X)}+\lambda}{\epsilon \lambda}\right)^{\alpha \nu}
$$

onde $\nu=\sup _{x \in K} \nu_{\lambda}\left(D_{x} f\right)<\infty$.

Notemos que, da compacidade de $K$ e da diferenciabilidade contínua de $g$ temos que, dado $\frac{1}{2 \lambda}-1-\epsilon>\eta>0$, existe $r_{0}>0$ tal que

$$
g\left(B_{r}(x)\right) \subset g(x)+D_{x} g\left(B_{r}(0)\right)+B_{\eta \lambda r}(0), 0<r<r_{0} .
$$

Consequentemente, existem $y_{i} \in D_{x} g\left(B_{r}(0)\right), 1 \leqslant i \leqslant \bar{N}_{\epsilon}$, tais que

$$
g\left(B_{r}(x)\right) \subset g(x)+\bigcup_{i=1}^{\bar{N}_{\epsilon}} B_{(1+\epsilon) \lambda r}\left(y_{i}\right)+B_{\eta \lambda r}(0)=g(x)+\bigcup_{i=1}^{\bar{N}_{\epsilon}} B_{(1+\epsilon+\eta) \lambda r}\left(y_{i}\right), 0<r<r_{0} .
$$


Como uma consequência imediata, temos que

$$
\tilde{N}\left((1+\epsilon+\eta) \lambda r, g\left(B_{r}(x)\right)\right) \leqslant \lambda_{1}:=(\nu+1)^{\alpha \nu}\left(\frac{D+\lambda}{\epsilon \lambda}\right)^{\alpha \nu}
$$

Então, se $K$ pode ser coberto por bolas $B_{r}\left(x_{1}\right), \cdots, B_{r}\left(x_{n}\right)$, com $x_{i} \in K, 1 \leqslant i \leqslant n$, segue que $K \subset g(K) \subset \cup_{i=1}^{n} g\left(B_{r}\left(x_{i}\right)\right)$. Pela desigualdade acima $g_{r}\left(B_{r}\left(x_{i}\right)\right)$ pode ser coberto por menos do que $\lambda_{1}$ bolas de raio $(1+\epsilon+\eta) \lambda r$. Portanto $K$ pode ser coberto por $\lambda_{1} n$ bolas de raio $(1+\epsilon+\eta) \lambda r$. Em outras palavras,

$$
\tilde{N}((1+\epsilon+\eta) \lambda r, K) \leqslant \lambda_{1} N(r, K) \leqslant \lambda_{1} \tilde{N}\left(\frac{r}{2}, K\right)
$$

para todo $0<r<r_{0}$. Então,

$$
\tilde{N}(2(1+\epsilon+\eta) \lambda r, K) \leqslant \lambda_{1} \tilde{N}(r, K)
$$

para todo $0<r<\frac{r_{0}}{2}$.

Ainda mais, se $0<r<(1+\epsilon+\eta) \lambda r_{0}$ podemos escrever $r=(2(1+\epsilon+\eta) \lambda)^{k} \bar{r}$ com $(1+\epsilon+\eta) \lambda r_{0} \leqslant \bar{r}<\frac{r_{0}}{2}, k \geqslant 1$, e aplicando a última desigualdade

$$
\tilde{N}(r, K)=\tilde{N}\left((2(1+\epsilon+\eta) \lambda)^{k} \bar{r}, K\right) \leqslant \lambda_{1}^{k} \tilde{N}(\bar{r}, K) \leqslant \lambda_{1}^{k} \tilde{N}\left((1+\epsilon+\eta) \lambda r_{0}, K\right)
$$

e então,

$$
\begin{gathered}
\frac{\log \tilde{N}(r, K)}{\log (1 / r)} \leqslant \frac{k \log \lambda_{1}+\log \tilde{N}\left((1+\epsilon+\eta) \lambda r_{0}, K\right)}{k \log \left(\frac{1}{2(1+\epsilon+\eta) \lambda}\right)+\log \left(\frac{1}{\bar{r}}\right)} \\
=\frac{\log \lambda_{1}}{\log \left(\frac{1}{2(1+\epsilon+\eta) \lambda}\right)+\frac{1}{k} \log \left(\frac{1}{\bar{r}}\right)}+\frac{\frac{1}{k} \log \left(\tilde{N}\left((1+\epsilon+\eta) \lambda r_{0}, K\right)\right)}{\log \left(\frac{1}{2(1+\epsilon+\eta) \lambda}\right)+\frac{1}{k} \log \left(\frac{1}{\bar{r}}\right)} .
\end{gathered}
$$

Notando que $\frac{1}{k} \leqslant \frac{\log \left(\frac{1}{2(1+\epsilon+\eta) \lambda}\right)}{\log \left(\frac{1}{r}\right)}$ e tomando que limsup quando $r \rightarrow 0$ obtemos

$$
c(K) \leqslant \frac{\log \lambda_{1}}{\log (1 / 2(1+\epsilon+\eta) \lambda)}
$$

Como esta desigualdade vale para todo $\frac{1}{2 \lambda}-1-\epsilon>\eta>0$ e $\lim _{\eta \rightarrow 0}(1+\epsilon+\eta) \lambda=(1+\epsilon) \lambda$ 
obtemos

$$
c(K) \leqslant \frac{\log \lambda_{1}}{\log \left(\frac{1}{2(1+\epsilon) \lambda}\right)}=\frac{\log \left[(\nu+1)^{\alpha \nu}\left(\frac{D+\lambda}{\epsilon \lambda}\right)^{\alpha \nu}\right]}{\log \left(\frac{1}{2(1+\epsilon) \lambda}\right)}
$$

e isto é precisamente o que pretendíamos mostrar.

A seguir mostraremos uma aplicação interessante dos resultados acima que mostram a compatibilidade da limitação obtida no teorema anterior. Ela diz que podemos tomar $\lambda$ tão pequeno quanto quisermos sem alterarmos $\nu, \nu$ é um limitante superior da dimensão fractal de $K$.

Corolário 3.3.11. Seja $X$ um espaço de Banach e assuma que $T \in \mathcal{C}^{1}(X)$ é tal que $\left\{T^{n}\right.$ : $n \geqslant 0\}$ tem um atrator global $\mathcal{A}$ e $D_{x} T$ tem posto finito $\nu(x) \operatorname{com} \sup _{x \in \mathcal{A}} \nu(x):=\nu<\infty$. Então,

$$
c(\mathcal{A}) \leqslant \alpha \nu
$$

onde $\alpha=1$ (ou 2) se $X$ for real (ou complexo).

Demonstração: Claramente, para cada $\lambda>0$ e $x \in \mathcal{A}, D_{x} T \in \mathcal{L}_{\frac{\lambda}{2}}(X)$ para todo $\lambda>0$. Consequentemente, para cada $0<\lambda<\frac{1}{2}$

$$
c(\mathcal{A}) \leqslant \alpha \nu \frac{\log \left((\nu+1) \frac{D}{\lambda}\right)}{\log \left(\frac{1}{2 \lambda}\right)} .
$$

Tomando o limite quando $\lambda \rightarrow 0$ temos que $c(\mathcal{A}) \leqslant \alpha \nu$.

Corolário 3.3.12. Seja $L, K \in \mathcal{C}^{1}(X)$. Se $T=L+K$, assuma que o semigrupo discreto $\left\{T^{n}: n \in \mathbb{N}\right\}$ tem um atrator global $\mathcal{A}$. Assuma que $K$ tem posto finito em $\mathcal{A} ;$ isto é, $R\left(D_{x} K\right) \subset Y(x)$ onde $Y(x)$ é subespaço de $X$ com $\sup _{x \in \mathcal{A}} \operatorname{dim}(Y(x)):=\nu<\infty$, e que $L$ satisfaz

$$
\sup _{x \in \mathcal{A}}\left\|D_{T^{n-1}(x)} L \circ \cdots \circ D_{x} L\right\| \leqslant c(n), n \in \mathbb{N},
$$

onde $c(n) \stackrel{n \rightarrow \infty}{\longrightarrow} 0$. Então, se $\sup _{x \in \mathcal{A}} \operatorname{dim}\left(D_{x} K\right)=\nu<\infty$, então

$$
c(\mathcal{A}) \leqslant \alpha \nu
$$

onde $\alpha=1$ (ou 2) se $X$ é real (ou complexo). 
Demonstração: Da demonstração anterior podemos fazer $\lambda$ tão pequeno quanto desejarmos. De fato, somente precisamos garantir que mudando $\lambda$ não alteramos $\nu$. Isto segue do fato de que

$$
\begin{aligned}
D_{x} T^{n} & =D_{T^{n-1}(x)} T \circ \cdots \circ D_{x} T=\left(D_{T^{n-1}(x)} L+D_{T^{n}-1(x)} K\right) \circ \cdots \circ\left(D_{x} l+D_{x} K\right) \\
& =D_{T^{n-1}(x)} L \circ \cdots \circ D_{x} L+K_{n}:=L_{n}+K_{n}
\end{aligned}
$$

onde $K_{n}$ é um operador compacto com posto menor ou igual a $\nu$. Claramente, existe $Z_{n}$ subespaço de $X$ tal que $\operatorname{dim}\left(Z_{n}\right) \leqslant \nu$ e

$$
\operatorname{dist}\left(D_{x} T^{n}\left[B_{1}^{x}(0), D_{x} T^{n}\left[B_{1}^{Z_{n}}(0)\right]\right]\right) \leqslant\left\|D_{T^{n-1}(x)} L \circ \cdots \circ D_{x} L\right\|_{\mathcal{L}(X)} \leqslant c(n) .
$$

Portanto, dado $\lambda>0$ mantendo $\nu$ e tomando $n$ grande podemos garantir que $D_{x} T^{n} \in \mathcal{L}_{\lambda}$ $\operatorname{com} \nu_{\lambda}\left(D_{x} T^{n}\right) \leqslant \nu$.

Como $T^{n}(\mathcal{A})=\mathcal{A}$ temos que

$$
c(\mathcal{A}) \leqslant \alpha \nu \frac{\log \left((\nu+1) \frac{D}{\lambda}\right)}{\log \left(\frac{1}{2 \lambda}\right)},
$$

para cada $0<\lambda<\frac{1}{2}$. Fazendo $\lambda \rightarrow 0$ obtemos que

$$
c(\mathcal{A}) \leqslant \alpha \nu
$$

e isto completa a prova. 


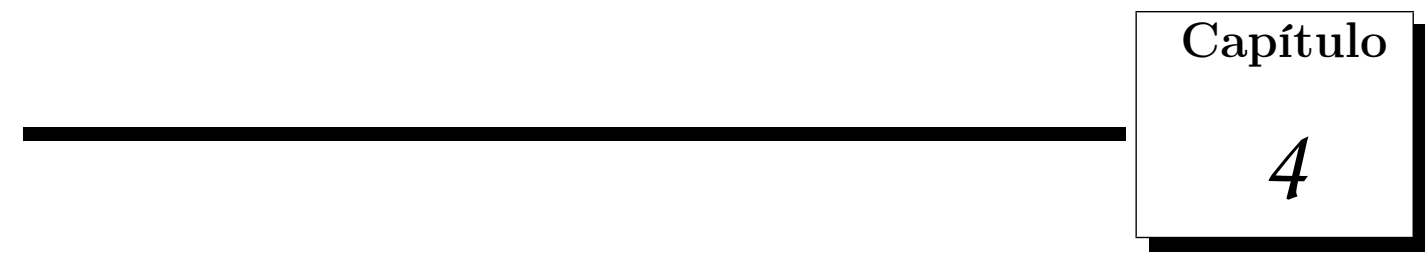

\section{Construção de atratores exponenciais}

\section{fractais}

A atração exponencial é uma propriedade muito importante e traz consigo muitas outras propriedades desejadas para aplicações. Claramente, a atração exponencial nos garante que o transiente (tempo decorrido até que as soluções sejam indistinguíveis daquelas no atrator) é "pequeno". Isto pode ter importância fundamental para tornar viável a análise numérica de tais sistemas.

Já vimos que alguns semigrupos gradient-like possuem esta propriedade e que dela decorrem propriedades importantes como a continuidade de atratores sob perturbação.

Infelizmente, a atração exponencial falha em diversas classes de semigrupos discretos (veremos exemplos a seguir). Neste capítulo, seguindo Eden-Foias-Nicolaenko-Temam [9], nos dedicamos a corrigir e melhorar os resultados apresentados e construiremos os chamados atratores exponenciais fractais para aplicações $S: X \rightarrow X$ Lipschitz contínuas em um subconjunto $X$ compacto em um espaço de Hilbert. Esta nova classe de atratores engloba a classe dos atratores exponenciais, e é construída simplesmente retirando a hipótese da invariância e pedindo simplesmente a invariância positiva do conjunto, mas ainda sim, pedindo que este novo atrator tenha dimensão fractal finita e, logicamente, tenha atração exponencial. No que segue vamos construir de fato atratores exponenciais fractais.

Consideremos $X$ um subconjunto compacto e conexo de um espaço de Hilbert $\mathcal{H}$ e $S$ uma aplicação Lipschitz contínua de $X$ em $X$ e denotemos a constante de Lipschitz de $S$ 
em $X$ por

$$
\operatorname{Lip}_{X}(S)=L
$$

Se $S$ está restrito à $X$, então o semigrupo $\left\{S^{n}: n \in \mathbb{N}\right\}$ possui um atrator global $\mathcal{A}$ dado por

$$
\mathcal{A}=\cap_{n \in \mathbb{N}} S^{n} X
$$

Já sabemos que $\mathcal{A}$ atrai todas as órbitas de $X$, isto é, a semi-distância de Hausdorff $\operatorname{dist}_{H}\left(S^{n} X, \mathcal{A}\right)$ tende a zero, quando $n$ tende a infinito. Entretanto, a taxa de convergência deste atrator não é controlada exponencialmente, como mostram os seguintes exemplos:

Exemplo 1: Seja $H=\mathbb{R}$, e defina $S:[0,1] \rightarrow[0,1]$ por

$$
S x=\frac{x}{1+x}, \text { para todo } x \in[0,1]
$$

Então $\mathcal{A}=\{0\}$, mas a taxa de convergência para $\mathcal{A}$ é polinomial. De fato, $S^{n} x=\frac{x}{1+n x}$ e portanto

$$
\left|S^{n} x\right|=\left|\frac{x}{1+n x}\right| \leqslant\left|\frac{1}{1+n x}\right|
$$

que claramente tem taxa de convergência polinomial.

Exemplo 2: Considere a equação diferencial $\dot{u}=-(u-1)^{2}$ no intervalo $[1, \infty) . O$ atrator para este problema é $\mathcal{A}=\{1\}$, pois $\dot{u}<0$. Resolvendo esta equação chegamos em $u(t)=1+\frac{u(0)-1}{1+(u(0)-1) t}$, para todo $t \in[1, \infty)$ e $u(0) \geqslant 1$. Mas então $|u(t)-1| \leqslant \frac{u(0)-1}{1+(u(0)-1) t}$, ou seja, a atração para este problema não é exponencial. O semigrupo discreto definido por $S u(0)=u(1)$ tem propriedades de atração análogas àquelas do Exemplo 1.

Para resolver esta deficiência nestes atratores, vamos introduzir o conceito de atrator exponencial fractal.

Definição 4.0.13. Um conjunto compacto $\mathcal{M}$ é chamado um atrator exponencial fractal para $(S, X)$ se $\mathcal{A} \subseteq \mathcal{M} \subseteq X$ e

i) $S \mathcal{M} \subseteq \mathcal{M}$,

ii) $c(\mathcal{M})<\infty$; isto é, $\mathcal{M}$ tem dimensão fractal finita,

iii) existem constantes positivas $c_{0}$ e $c_{1}$ tais que

$$
\operatorname{dist}_{H}\left(S^{n} X, \mathcal{M}\right) \leqslant c_{0} e^{-c_{1} n}, \quad \forall n \geqslant 1
$$


Definição 4.0.14. Fixemos $\alpha \in \mathbb{R}$. Dizemos que $S$ tem a propriedade squeezing em $X$ se para algum $\delta \in\left(0, \frac{1}{2}\right)$ existe uma projeção ortogonal $P=P(\delta)$ de posto igual a $N_{0}(\delta)$ tal que para $u, v \in X$, tais que

$$
\|S u-S v\|_{H}>\sqrt{1+\alpha^{2}}\|P(S u-S v)\|_{H}
$$

então

$$
\|S u-S v\|_{H} \leqslant \delta\|u-v\|
$$

Para podermos construir atratores exponenciais fractais, vamos considerar subconjuntos de $S^{k}(X)$ que são maximais com respeito à propriedade do cone

$$
\|u-v\|_{H} \leqslant \sqrt{1+\alpha^{2}}\|P(u-v)\|_{H}
$$

Para isto, definimos

$$
Z=S\left(\bar{B}_{r}^{H}(a) \cap X\right)
$$

Lema 4.0.15. Existe um subconjunto $E$ de $Z$ que é maximal com relação à propriedade do cone

$$
\|u-v\|_{H} \leqslant \sqrt{1+\alpha^{2}}\|P(u-v)\|_{H}, \quad \forall u, v \in E .
$$

Demonstração: Seja $\mathcal{V}$ a família de todos os subconjuntos $A \subset Z$ que satisfazem a propriedade do cone. Claramente $\mathcal{V}$ é não-vazio, uma vez que $\{x\} \in \mathcal{V}$ para cada $x \in Z$, e é parcialmente ordenado pela relação de inclusão. Seja $\mathcal{G}$ um subconjunto totalmente ordenado de $\mathcal{V}$. Então, $\cup_{A \in \mathcal{G}} A \in \mathcal{V}$ é um limitante superior para $G$. Segue do Lema de Zorn que existe um elemento maximal $E \in V$.

Notemos que o conjunto $E$ é fechado, e logo, compacto. Além disso, $P$ é injetiva em $E$. Portanto, toda cobertura de $E$ pode ser obtida através de uma cobertura de $P E$. Usando a injetividade de $P$ em $E$, podemos estimar o número de $\rho$-bolas necessárias para cobrir $Z$ em termos de $N_{0}$ e $\rho$.

Lema 4.0.16. Para cada $\rho>0$, existe um número $K \in \mathbb{N}$ tal que para todo subconjunto $B \subset P E$ com mais que $K$ elementos existem $x, y \in B$, com $x \neq y$, tal que $\|x-y\|<\rho$.

Demonstração: Suponhamos por absurdo que seja falso, isto é, para cada $n \in N$ existe 
um subconjunto $B_{n} \subset P E$ com mais do que $n$ elementos tal que

$$
\|x-y\| \geqslant \rho, \text { para todo } x, y \in B_{n}, \operatorname{com} x \neq y
$$

Assim, para cada $n$, conseguimos um conjunto $\left\{x_{1}^{n}, \ldots, x_{n}^{n}\right\}$ tais que $\left\|x_{i}^{n}-x_{j}^{n}\right\| \geqslant \rho$, para todo $1 \leqslant i, j \leqslant n$ com $i \neq j$. Vamos construir agora uma sequência que não possui nenhuma subsequência convergente, contrariando a compacidade de $P E$.

Temos a sequência $\left\{x_{1}^{n}\right\}_{n \in N} \subset P E$ e como $P E$ é compacto, $\left\{x_{1}^{n}\right\}_{n \in N}$ possui uma subsequência $\left\{x_{1}^{n_{k}}\right\}$ convergente para, digamos, $x_{1}^{\infty}$. Agora, temos a sequência $\left\{x_{2}^{n}\right\}_{n \in N} \subset$ $P E$, e considere $\left\{x_{2}^{n_{k}}\right\}_{n \in N}$. Da mesma forma $\left\{x_{2}^{n_{k}}\right\}_{n \in N}$ possui uma subsequência $\left\{x_{2}^{n_{k_{j}}}\right\}_{n \in N}$ convergente para $x_{2}^{\infty}$, e como $\left\|x_{1}^{n_{k_{j}}}-x_{2}^{n_{k_{j}}}\right\| \geqslant \rho$, para todo $j \in \mathbb{N}$ temos $\left\|x_{1}^{\infty}-x_{2}^{\infty}\right\| \geqslant \rho$.

Indutivamente, construímos assim uma sequência $\left\{x_{k}^{\infty}\right\}_{k \in \mathbb{N}}$ que não possui nenhuma subsequência convergente.

Lema 4.0.17. Para qualquer $2 \delta<\theta<1$, existe uma cobertura de $Z$, definido em (4.0.2), por $K_{0} \theta r$-bolas, centradas em $y_{j} \in E$, com $j=1, \ldots, K_{0}$; mais ainda, $K_{0}$ pode ser estimado por

$$
K_{0} \leqslant\left(\frac{3 L \sqrt{1+\alpha^{2}}}{\theta-2 \delta}+1\right)^{N_{0}}
$$

onde $\delta$ é como dado na Definição (4.0.14).

Demonstração: Como $P E \subseteq P Z \subseteq P \mathcal{H}$, temos

$$
\operatorname{diam}(P E) \leqslant \operatorname{diam}(P Z)=\sup _{u, v \in Z}|P u-P v| \leqslant \sup _{u, v \in Z}|u-v|=\operatorname{diam}(Z)
$$

pois como $P$ é ortogonal, $\|P\|=1$. Assim, se $\Omega=\bar{B}_{r}^{H}(a) \cap X \bar{B}_{r}^{H}(a) \cap X$

$$
\begin{aligned}
\operatorname{diam}(P E) \leqslant \operatorname{diam}(Z) & =\sup _{u, v \in Z}\|u-v\|=\sup _{x, y \in \Omega}\|S x-S y\| \\
& \leqslant \sup _{x, y \in \Omega} L\|x-y\| \leqslant 2 L r .
\end{aligned}
$$

Agora, dado $\rho>0$ arbitrário, podemos cobrir $P E$ com $K_{0}$ bolas $B_{\rho}^{P \mathcal{H}}\left(P y_{j}\right)$ com $y_{j} \in E$ e $j=1, \ldots, K_{0}$, uma vez que $P E$ é compacto. Note que a injetividade de $P$ em $E$ garante que os pontos $P y_{j}$ são pontos distintos em $P \mathcal{H}$ para diferentes índices $j$. Para podermos estimar $K_{0}$, usaremos o fato de que $\operatorname{diam}(P E) \leqslant 2 L r$. Observemos que, se $K$ é o número máximo de pontos em $P E$ separados por uma distância maior que $\rho$, então as $\rho$-bolas 
centradas nestes pontos cobrem $P E$, $\log 0 K_{0} \leqslant K$. Por outro lado, $\frac{\rho}{2}$-bolas centradas em $P y_{j}, j=1, \ldots, K$ são disjuntas e

$$
\cup_{j=1}^{K} B_{\frac{\rho}{2}}^{P \mathcal{H}}\left(P y_{j}\right) \subseteq \mathcal{O}_{\frac{\rho}{2}}^{P \mathcal{H}}(P E):=\left\{y \in P \mathcal{H}: \operatorname{dist}(y, P E)<\frac{\rho}{2}\right\}
$$

$\operatorname{Como} \operatorname{diam}\left(B_{\frac{\rho}{2}}^{P \mathcal{H}}(P E)\right) \leqslant 2 L r+\rho$, por uma comparação de volumes obtemos

$$
K \omega_{N_{0}}\left(\frac{\rho}{2}\right)^{N_{0}} \leqslant \omega_{N_{0}}\left(L r+\frac{\rho}{2}\right)^{N_{0}}
$$

onde $\omega_{N_{0}}$ é o volume da bola unitária em $\mathbb{R}^{N_{0}}$. Consequentemente, obtemos

$$
K_{0} \leqslant K \leqslant\left(\frac{2 L r}{\rho}+1\right)^{N_{0}}
$$

Afirmamos que simplesmente expandindo o raio desta cobertura de $P E$ podemos obter uma cobertura para $E$. De fato, dada esta cobertura de $P E$, temos

$$
\left\|P y-P y_{j}\right\|_{H}<\left\|y-y_{j}\right\|_{H} \rightarrow\left\|y-y_{j}\right\|_{H}<\sqrt{2} \rho \text {, para todo } y \in E,
$$

e portanto, $\mathcal{U}=\left\{B_{\sqrt{2} \rho}^{H}\left(y_{j}\right)\right\}_{j=1}^{K_{0}}$ é uma cobertura de $E$.

Nos resta mostrar que conseguimos de fato uma cobertura para $Z$. Se $y \in E$, então $P y \in P E$ e assim $\left\|P y-P y_{i}\right\|<\rho$, para algum $i \in\left\{1,2, \ldots, K_{0}\right\}$. Se, por outro lado, $z \in Z \backslash E$, enão, por definição de $Z$ e $E$, existem $u, v \in \bar{B}_{r}(a) \cap X$ tais que

$$
z=S u \text { e } y=S v \in E, \operatorname{com}\|z-y\|>\sqrt{1+\alpha^{2}}\|P(z-y)\|
$$

Segue da propriedade de squeezing que

$$
\|z-y\|=\|S u-S v\| \leqslant \delta\|u-v\| \leqslant 2 r \delta .
$$

Como $y \in E$, existe $y_{j} \in E$ tal que $\left\|P y-P y_{j}\right\|<\rho$ e portanto $\left\|y-y_{j}\right\|<\sqrt{1+\alpha^{2}} \rho$, assim

$$
\left\|z-y_{j}\right\| \leqslant\|z-y\|+\left\|y-y_{j}\right\| \leqslant 2 r \delta+\sqrt{1+\alpha^{2}} \rho<\theta r
$$


se escolhermos $\rho=\frac{2(\theta-2 \delta) r}{3 \sqrt{1+\alpha^{2}}}$. Portanto,

$$
K_{0} \leqslant\left(\frac{3 L \sqrt{1+\alpha^{2}}}{\theta-2 \delta}+1\right)^{N_{0}}
$$

Notemos também que $\sqrt{1+\alpha^{2}} \rho<\theta r$, e assim $\mathcal{U}=\left\{B_{\theta r}\left(y_{j}\right)\right\}_{j=1}^{K_{0}}$ é uma cobertura com as propriedades desejadas.

Observação 4.0.18. $\quad$ i) Quando $\alpha=1$ a estimativa pode ser simplificada, tomando $\rho=\frac{2(\theta-2 \delta) r}{3}$, e toma a seguinte forma

$$
K_{0} \leqslant\left(\frac{3 L}{\theta-2 \delta}+1\right)^{N_{0}}
$$

ii) Quando $\delta$ pode ser tomado no intervalo $\left(0, \frac{1}{4}\right)$, podemos escolher $2 \delta<\theta=4 \delta<1$, e a estimativa fica

$$
K_{0} \leqslant\left(\frac{3 L \sqrt{1+\alpha^{2}}}{2 \delta}+1\right)^{N_{0}} \leqslant\left(\frac{2 L \sqrt{1+\alpha^{2}}}{\delta}+1\right)^{N_{0}} .
$$

Agora vamos proceder indutivamente, a fim de construir uma cobertura para $S^{k+1}(X)$, para $k \geqslant 1$. Para começar este processo, seja $R>0$ e $a \in X$ tais que $X \subseteq \bar{B}_{R}(a)$. Denotemos por $E_{1}$ um subconjunto maximal de $S\left(X \cap \bar{B}_{R}(a)\right)=S(X)$ para a propriedade do cone 4.0.1. Então do Lema 4.0.17, existem $\left\{a_{j_{1}}\right\}_{j_{1}=1}^{K_{0}} \subset E_{1}$ tais que

$$
S(X)=S\left(X \cap \bar{B}_{R}(a)\right) \subseteq \bigcup_{j_{1}=1}^{K_{0}} \bar{B}_{\theta R}\left(a_{j_{1}}\right) \cap S(X),
$$

onde $K_{0}$ pode ser estimado por 4.0 .3 .

Vamos agora construir uma cobertura para $S^{2}(X)$. Para isto, seja $E_{2 ; j_{1}}$ o subconjunto maximal de $S\left(\bar{B}_{\theta R}\left(a_{j_{1}}\right) \cap X\right)$ para a propriedade do cone, para $j_{1}=1, \ldots, K_{0}$. Como no Lema 4.0.17, podemos cobrir cada um destes compactos com $K_{1}$ bolas $\bar{B}_{\theta^{2} R}\left(a_{j_{1} ; j_{2}}\right) \cap S(X)$, com $j_{2}=1, \ldots, K_{1}$. Tomando na demonstração do Lema $4.0 .17 r=\theta R$ e $\rho=\frac{2(\theta-2 \delta) \theta R}{3 \sqrt{1+\alpha^{2}}}$ 
temos

$$
K_{1} \leqslant\left(\frac{3 L \sqrt{1+\alpha^{2}}}{\theta-2 \delta}+1\right)^{N_{0}}
$$

Assim, usando a mesma estimativa para $K_{0}$ e $K_{1}$, podemos tomar $K_{1}=K_{0}$. Ainda mais, notamos que

$$
\bigcup_{j_{1}=1}^{K_{0}} E_{2 ; j_{1}} \subset \bigcup_{j_{1}=1}^{K_{0}} S\left(\bar{B}_{\theta R}\left(a_{j_{1}}\right) \cap S X\right) \subset S^{2} X
$$

e

$$
S^{2} X \subset \bigcup_{j_{1}=1}^{K_{0}} S\left(\bar{B}_{\theta R}\left(a_{j_{1}}\right) \cap S X\right) \subset \bigcup_{j_{1}, j_{2}=1}^{K_{0}} \bar{B}_{\theta^{2} R}\left(a_{j_{1} ; j_{2}}\right) \cap S^{2} X .
$$

Procedendo iterativamente podemos cobrir $S^{k+1}(X)$, isto é, existem $a_{j_{1} ; j_{2} ; \ldots ; j_{k}} \in E_{k+1 ; j_{1} ; \ldots ; j_{k}}$, onde $E_{k+1 ; j_{1} ; \ldots ; j_{k}}$ é o subconjunto maximal de $S\left(\bar{B}_{\theta^{k} R}\left(a_{j_{1} ; \ldots ; j_{k}}\right) \cap S^{k}(X)\right)$ com relação à propriedade do cone, tais que

$$
\bigcup_{j_{1}, \ldots, j_{k}=1}^{K_{0}} E_{k+1 ; j_{1}, \ldots, j_{k}} \subset \bigcup_{j_{1}, \ldots, j_{k}=1}^{K_{0}} S\left(\bar{B}_{\theta^{k} R}\left(a_{j_{1} ; \ldots ; j_{k}}\right) \cap S^{k}(X)\right) \subset S^{k+1}(X)
$$

$\mathrm{e}$

$$
S^{k+1}(X) \subset \bigcup_{j_{1}, \ldots, j_{k}+1=1}^{K_{0}} \bar{B} \theta^{k+1} R\left(a_{j_{1}, \ldots, j_{k+1}}\right) \cap S^{k+1}(X) .
$$

Vamos agora passar de fato para a construção de um atrator exponencial fractal $\mathcal{M}$ para $S$. Para tanto, vamos nos munir de vários lemas, até chegarmos de fato em uma expressão para $\mathcal{M}$. Mas antes, vamos definir

$$
E^{(k)}=\bigcup_{j_{1}, \ldots, j_{k}=1}^{K_{0}}\left\{a_{j_{1}, \ldots, j_{k}}\right\}
$$

Lema 4.0.19. O atrator global $\mathcal{A}$ tem dimensão fractal $c(\mathcal{A})$ finita, que pode ser estimada $\operatorname{por} \kappa(\mathcal{A})=\frac{\log K_{0}}{\log (1 / \theta)}$.

Demonstração: Seja $N(\rho, \mathcal{A})$ o número mínimo de bolas de raio $\rho$ que são necessárias para cobri $\mathcal{A}$, que é compacto. Então, se $\theta<1$, usando a idéia do Lema 4.0.17, para $X=\mathcal{A}$, e lembrando que $s(\mathcal{A})=\mathcal{A}$, obtemos uma cobertura de $\mathcal{A}$ por bolas de raio $\theta \rho$, 
onde

$$
N(\theta \rho, \mathcal{A})=N(\theta \rho, S(\mathcal{A})) \leqslant K_{0} N(\rho, \mathcal{A})
$$

Agora, por meio de iterações,

$$
N\left(\theta^{j} \rho, \mathcal{A}\right) \leqslant K_{0}^{j} N(\rho, \mathcal{A}), \text { para todo } j \in \mathbb{N} \text {. }
$$

Se escolhermos $\epsilon>0$ tal que $\theta^{j+1} \rho<\epsilon \leqslant \theta^{j} \rho$, e usando o fato de que $N(\epsilon, \mathcal{A})$ é uma função decrescente de $\epsilon$ obtemos

$$
N(\epsilon, \mathcal{A}) \leqslant N\left(\theta^{j+1} \rho, \mathcal{A}\right) \leqslant K_{0}^{j+1} N(\rho, \mathcal{A})
$$

Como $\log (1 / \epsilon) \geqslant-\log \left(\theta^{j} \rho\right)$, temos que

$$
\frac{\log N(\epsilon, \mathcal{A})}{\log (1 / \epsilon)} \leqslant \frac{\log \left(K_{0}^{j+1} N(\rho, \mathcal{A})\right)}{-\log \left(\theta^{j} \rho\right)}
$$

Portanto,

$$
c(\mathcal{A})=\limsup _{\epsilon \rightarrow 0} \frac{\log N(\epsilon, \mathcal{A})}{\log (1 / \epsilon)} \leqslant \lim _{j \rightarrow \infty} \frac{\log \left(K_{0}^{j+1} N(\rho, \mathcal{A})\right)}{-\log \left(\theta^{j} \rho\right)}=\kappa(\mathcal{A}) .
$$

Lema 4.0.20. Defina $C_{\infty}=\overline{\cup_{k=1}^{\infty} E^{(k)}}$, então

$$
c\left(C_{\infty}\right) \leqslant \kappa(\mathcal{A})
$$

Demonstração: De fato, sabemos que

$$
C_{\infty} \subset \overline{\cup_{k=1}^{n} E^{(k)}} \cup S^{n+1}(X),
$$

e se $\epsilon>0$ é tal que $\theta^{n+1}<\epsilon \leqslant \theta^{n}$ então existe $\rho \in[1,1 / \theta]$ tal que $\epsilon=\theta^{n+1} \rho$. Assim 


$$
\begin{aligned}
N\left(\epsilon, C_{\infty}\right) \leqslant & \sum_{k=1}^{n} N\left(\epsilon, E^{(k)}\right)+N\left(\epsilon, S^{n+1}(X)\right) \leqslant \\
\leqslant & \sum_{k=1}^{n} K_{0}^{k}+K_{0}^{n+1} N(\rho, X) \leqslant \\
& \leqslant K_{0}^{n+1}(n+N(\rho, X)),
\end{aligned}
$$

pois cada $E^{(k)}$ é um conjunto finito com $K_{0}^{k}$ pontos e $K_{0} \leqslant 1$. Assim

$$
\frac{\log N\left(\epsilon, c\left(C_{\infty}\right)\right)}{\log (1 / \epsilon)} \leqslant \frac{\log \left[K_{0}^{n+1}(n+N(\rho, X))\right]}{-\log \left(\theta^{n} \rho\right)}
$$

e portanto $c\left(C_{\infty}\right) \leqslant \kappa(\mathcal{A})$.

Lema 4.0.21. Se $W$ é um subconjunto compacto de $X$ e $c(W) \leqslant \kappa(\mathcal{A})$ então

$$
\Gamma_{\infty}^{W}=\overline{\bigcup_{j=0}^{\infty} S^{j}(W)}
$$

tem dimensão fractal finita e $c\left(\Gamma_{\infty}^{W}\right) \leqslant \kappa(\mathcal{A})$.

Demonstração: Notemos que

$$
\Gamma_{\infty}^{W} \subseteq \overline{\bigcup_{j=0}^{n} S^{j}(W)} \cup S_{n+1}(X)
$$

Seja $\epsilon>0$ tal que $\theta^{n+1}<\epsilon \leqslant \theta^{n}$ e $\rho \in[1,1 / \theta]$ tal que $\theta^{n+1} \rho=\epsilon$. Logo

$$
\begin{aligned}
N\left(\epsilon, \Gamma_{\infty}^{W}\right) & \leqslant \sum_{j=0}^{n} N\left(\epsilon, S^{j}(W)\right)+K_{0}^{n+1} N(\rho, X) \leqslant \\
& \leqslant \sum_{j=0}^{n} K_{0}^{j} N\left(\epsilon \theta^{-j}, W\right)+K_{0}^{n+1} N(\rho, X) .
\end{aligned}
$$

Como $c(W) \leqslant \kappa(\mathcal{A})$, para todo $d>\kappa(\mathcal{A})$ existe $\delta_{0}=\delta_{0}(d)>0$ tal que $N(\delta, W)<\left(\frac{1}{\delta}\right)^{d}$ 
para $\delta \leqslant \delta_{0}$. Agora, para $\delta_{0}<\delta<1$ temos

$$
N(\delta, W) \leqslant N\left(\delta_{0}, W\right) \leqslant\left(\frac{1}{\delta_{0}}\right)^{d}=\left(\frac{\delta}{\delta_{0}}\right)^{d}\left(\frac{1}{\delta}\right)^{d}=c_{d}\left(\frac{1}{\delta}\right)^{d}
$$

onde $c_{d}=\left(\frac{\delta}{\delta_{0}}\right)^{d} \geqslant 1$. Assim, para qualquer $\delta \in(0,1)$ temos $N(\delta, W) \leqslant c_{d}\left(\frac{1}{\delta}\right)^{d}$, e a estimativa acima assume a forma

$$
N\left(\epsilon, \Gamma_{\infty}^{W}\right) \leqslant c_{d}\left(\frac{1}{\epsilon}\right)^{d} \sum_{j=0}^{n}\left(K_{0} \theta^{d}\right)^{j}+K_{0}^{n+1} N(\rho, X) .
$$

Afirmamos que $K_{0} \theta^{d} \leqslant 1$, pois caso contrário, teríamos $\log K_{0}>d \log (1 / \theta)$, o que implica que $d<\kappa(\mathcal{A})$, contradizendo a escolha de $d$. Logo, temos

$$
N\left(\epsilon, \Gamma_{\infty}^{W}\right) \leqslant c_{d}\left(\frac{1}{\epsilon}\right)^{d} n+K_{0}^{n+1} N(\rho, X)
$$

Substituindo $n+1=\frac{\log (\rho / \epsilon)}{\log (1 / \theta)}$, temos

$$
\begin{aligned}
N\left(\epsilon, \Gamma_{\infty}^{W}\right) & \leqslant \frac{c_{d}}{\rho^{d}}\left(\frac{\rho}{\epsilon}\right)^{d} \frac{\log (\rho / \epsilon)}{\log (1 / \theta)}+N(\rho, X) K_{0}^{\frac{\log (\rho / \epsilon)}{\log (1 / \theta)}} \leqslant \\
& \leqslant \frac{c_{d}}{\rho^{d}}\left(\frac{\rho}{\epsilon}\right)^{d} \frac{\log (\rho / \epsilon)}{\log (1 / \theta)}+N(\rho, X)\left(\frac{\rho}{\epsilon}\right)^{\frac{\log K_{0}}{\log (1 / \theta)}} \leqslant \\
& \leqslant\left(\frac{\rho}{\epsilon}\right)^{d}\left[\frac{c_{d}}{\rho^{d}} \frac{\log (\rho / \epsilon)}{\log (1 / \theta)}+N(\rho, X)\right]
\end{aligned}
$$

pois $\kappa(\mathcal{A})<d$. Portanto

$$
c\left(\Gamma_{\infty}^{W}\right)=\limsup _{\epsilon \rightarrow 0} \frac{\log N\left(\epsilon, \Gamma_{\infty}^{W}\right)}{\log (1 / \epsilon)} \leqslant d,
$$

e como $d>\kappa(\mathcal{A})$ foi tomado arbitrário temos $c\left(\Gamma_{\infty}^{W}\right) \leqslant \kappa(\mathcal{A})$.

Definição 4.0.22. Sejam $\mathcal{A}$ o atrator global de $S$ e $\Gamma_{\infty}=\Gamma_{\infty}^{C_{\infty}}$. Defina $\mathcal{M}=\mathcal{A} \cup \Gamma_{\infty}$.

Segue diretamente da definição que $\mathcal{M}$ é fechado e $S(\mathcal{M}) \subseteq \mathcal{M}$. Agora o seguinte lema se refere à dimensão fractal de $\mathcal{M}$.

Lema 4.0.23. $c(\mathcal{M}) \leqslant \kappa(\mathcal{A})$. 
Demonstração: Como ambas $c(\mathcal{A})$ e $c\left(\Gamma_{\infty}\right)$ são majoradas por $\kappa(\mathcal{A})$, para qualquer $d>\kappa(\mathcal{A})$ existe $\epsilon_{0}>0$ tal que $\epsilon \leqslant \epsilon_{0}$ implica em

$$
N(\epsilon, \mathcal{A}) \leqslant(1 / \epsilon)^{d} \text { e } N\left(\epsilon, \Gamma_{\infty}\right) \leqslant(1 / \epsilon)^{d} .
$$

Portanto

$$
N(\epsilon, \mathcal{M}) \leqslant N(\epsilon, \mathcal{A})+N\left(\epsilon, \Gamma_{\infty}\right) \leqslant 2(1 / \epsilon)^{d} .
$$

Usando a definição de $c(\mathcal{M})$, temos

$$
c(\mathcal{M})=\limsup _{\epsilon \rightarrow 0} \frac{\log N(\epsilon, \mathcal{M})}{\log (1 / \epsilon)} \lim _{\epsilon \rightarrow 0} \frac{\log 2+d \log (1 / \epsilon)}{\log (1 / \epsilon)}=d .
$$

Portanto, $c(\mathcal{M}) \leqslant \kappa(\mathcal{A})$.

Lema 4.0.24. Para todo $x \in X$, $\operatorname{dist}_{H}\left(S^{k}(x), \mathcal{M}\right) \leqslant R \theta^{k}$.

Demonstração: Notemos que $S^{k}(x) \in S^{k}(X)$ e portanto existe $a_{j_{1}, \ldots, j_{k}}$ em $E^{(k)}$ tal que

$$
\left\|S^{k}(x)-a_{j_{1}, \ldots, j_{k}}\right\|<R \theta^{k}
$$

Como $E^{(k)} \subset \mathcal{M}$ por construção, segue que

$$
\operatorname{dist}_{H}\left(S^{k}(x), \mathcal{M}\right)=\inf _{m \in \mathcal{M}}\left\|S^{k}(x)-m\right\| \leqslant R \theta^{k}
$$

e como a escolha de $x$ é arbitária

$$
\operatorname{dist}_{H}\left(S^{k}(X), \mathcal{M}\right)=\sup _{x \in X} \inf _{m \in \mathcal{M}}\left\|S^{k}(x)-m\right\| \leqslant R \theta^{k}
$$

Construímos assim um atrator exponencial fractal $\mathcal{M}$ para $S$, o que nos dá o teorema principal deste capítulo

Teorema 4.0.25. O conjunto $\mathcal{M}=\mathcal{A} \cup \Gamma_{\infty}$ é um atrator exponencial fractal para a aplicação $S$. 


\section{Referências Bibliográficas}

[1] Babin, A.V., Vishik, M.I., Attractors in Evolutionary Equations Studies in Mathematics and its Applications 25, North-Holland Publishing Co., Amsterdam, 1992.

[2] Bollobás, B. Linear Analysis: Introductory Course. Cambridge Mathematical Textbooks, Cambridge University Press, Cambridge UK (1990).

[3] Brezis, H., Analyse Fonctionnelle: Théorie et applications. Collection Mathématiques appliquées pour la maîtrise, (2) (1987).

[4] Carvalho, A.N., Langa, J.A., An extension of the concept of gradient systems which is stable under perturbation. Journal of Differential Equations, 246 (7) 2646-2668 (2009)

[5] Carvalho, A.N., Langa, J.A., Robinson, J.C., Suárez, A., Characterization of nonautonomous attractors of a perturbed infinite-dimensional gradient system. Journal of Differential Equations, 236 (2007) 570-603.

[6] Carvalho, A.N., Langa, J.A., Robinson, J.C., Finite-dimensional global attractors in Banach spaces, Royal Society of Mathematics.

[7] Chepyzhov, V.V., Vishik, M.I. Attractors for Equations of Mathematical Physics (2002) (Providence, AMS Colloquium Publications vol. 49, A.M.S).

[8] Deimling, K. Nonlinear Functional Analysis, Springer-Verlag (1985).

[9] Eden, A., Foias, C., Nicolaenko, B., Temam, R.,Exponential Attractor for Dissipative Evolution Equations, Research in Applied Mathematics, John Wiley \& Sons (1994).

[10] Falconer, K.J., The geometry of fractal sets, Cambridge Tracts in Mathematics 85, Cambridger University Press (1985). 
[11] Folland, G.B., Real Analysis: Modern techniques and their applications, Pure \& Applied Mathematics, John Wiley \& Sons (1984).

[12] Hale, J.K., Asymptotic Behavior of Dissipative Systems, Mathematical Surveys and Monographs Number 25 (American Mathematical Society, Providence, RI) (1988).

[13] Hale, J.K., Raugel, G., Lower semicontinuity of attractors of gradient systems and applications, Ann. Mat. Pura Appl. 154 (4) (1989), 281-326.

[14] Kahane, J.P., Measures et dimensions, Turbulence and the Navier Stokes equation, Lecture Notes in Mathematics 565 Springer-Verlag, New York, (1976).

[15] Krasnosel'skii, M.A., Zabreiko, P.P. Geometrical methods of nonlinear analysis. Translated from the Russian by Christian C. Fenske. Grundlehren der Mathematischen Wissenschaften [Fundamental Principles of Mathematical Sciences], 263. Springer-Verlag, Berlin, 1984. xix +409 pp.

[16] Ladyzhenskaya, O.A., Attractors for semigroups and evolution equations, Leizioni Lincee, Cambridge Univ. Press, Campridge, UK (1991)

[17] Mañé, R. On the dimension of the compact invariant sets of certain non-linear maps, Lecture Notes in Mathematics 898 230-242 Springer-Verlag, New York, 1981.

[18] Munkres, J.R. Topology, Second Edition, Prentice Hall (2000).

[19] Robinson, J.C. Infinite-Dimensional Dynamical Systems, From Basic Facts to Actual Calculations, Cambridge University Pres, Cabridge UK (2001).

[20] Rudin, W., Principles of Mathematical Analysis, McGraw-Hill (1976).

[21] Temam, R. Infinite dimensional dynamical systems in mechanics and physics. New York: Springer. 


\section{Índice Remissivo}

absorção, 9

atração, 9

atrator

do tipo gradiente, 25

exponencial, 40

exponencial fractal, 72

global, 14

base de Auerbach, 58

conjunto

$\alpha$-limite, 8

$\mu^{*}$-mensurável, 49

$\omega$-limite, 8

estável, 38

instável, 23, 38

invariante, 9

negativamente invariante, 9

positivamente invariante, 9

dimensão

de Hausdorff, 51

fractal, 52

topológica, 48

distância de Banach-Mazur, 58

$\epsilon$-cadeia, 27

estrutura homoclínica, 30

função de Liapunov, 22 medida

de não-compacidade de Kuratowski, 9

exterior, 48

órbita

positiva, 6

global, 7

negativa, 7

para trás, 7

parcial, 6

ponto

de equilíbrio, 10

fixo, 10

recorrente por cadeias, 27

Princípio da Contração de Banach, 5

projeção, 54

propriedade

do cone, 73

squeezing, 73

semi-distância de Hausdorff, 9

semicontinuidade

inferior, 19

superior, 19

semigrupo discreto, 6

com atrator do tipo gradiente, 25

condicionalmente eventualmente compacto, 13 assintoticamente compacto, 12 
coletivamente assintoticamente compacto, 32

compacto dissipativo, 13

eventualmente compacto, 13

eventualmente limitado, 16

gradient-like, 28

gradiente, 22

limitado, 7

limitado dissipativo, 13

ponto dissipativo, 13

solução, 7

de equilíbrio, 10

estacionária, 10

global, 7

para trás, 7

Teorema do Gráfico Fechado, 5 